\title{
Tidal and rotational effects in the perturbations of hierarchical triple stellar systems
}

\section{Eccentric systems - the case of AS Camelopardalis}

\author{
T. Borkovits ${ }^{1}$, E. Forgács-Dajka ${ }^{2}$, and Zs. Regály ${ }^{3}$ \\ ${ }^{1}$ Baja Astronomical Observatory of Bács-Kiskun County, H-6500 Baja, Szegedi út, Pf. 766, Hungary, \\ e-mail: borko@alcyone.bajaobs.hu \\ ${ }^{2}$ Eötvös University, Department of Astronomy, H-1518 Budapest, Pf. 32, Hungary, \\ e-mail: E.Forgacs-Dajka@astro.elte.hu \\ ${ }^{3}$ Konkoly Observatory of HAS, H-1525 Budapest, Pf. 67, Hungary \\ e-mail: regaly@konkoly.hu \\ Received idate $_{i} /$ Accepted $_{\text {idate }} \underset{ }{ }$
}

\begin{abstract}
Aims. We study the perturbations of a relatively close third star on a tidally distorted eccentric eclipsing binary. We consider both the observational consequences of the variations of the orbital elements and the interactions of the stellar rotation with the orbital revolution in the presence of dissipation. We concentrate mainly on the effect of a hypothetical third companion on both the real, and the observed apsidal motion period. We investigate how the observed period derived mainly from some variants of the $\mathrm{O}-\mathrm{C}$ relates to the real apsidal motion period.

Methods. We carried out both analytical and numerical investigations and give the time variations of the orbital elements of the binary both in the dynamical and the observational reference frames. We give the direct analytical form of an eclipsing O-C affected simultaneously by the mutual tidal forces and the gravitational interactions with a tertiary. We also integrated numerically simultaneously the orbital and rotational equations for the possible hierarchical triple stellar system AS Camelopardalis.

Results. We find that there is a significant domain of the possible hierarchical triple system configurations, where both the dynamical and the observational effects tend to measure longer apsidal advance rate than is expected theoretically. This happens when the mutual inclination of the close and the wide orbits is large, and the orbital plane of the tertiary almost coincides with the plane of the sky. We also obtain new numerical results on the interaction of the orbital evolution and stellar rotation in such triplets. The most important fact is that resonances might occur as the stellar rotational rate varies during the dissipation-driven synchronization process, for example in the case when the rotational rate of one of the stars reaches the average Keplerian angular velocity of the orbital revolution.
\end{abstract}

Key words. methods: analytical - methods: numerical - celestial mechanics - stars: binaries: close - stars: individual: AS Cam

\section{Introduction}

In a previous paper (Borkovits et al. 2004), we introduced a new numerical code which integrated simultaneously the orbital equations of hierarchical triple systems, including the tidal and dissipative terms, and the Eulerian equations of stellar rotation. First we applied the method for the Algol system itself. In this triple the inner binary had an almost circular orbit with approximately synchronized stellar rotation (another application of an earlier version of the code, not including tidal dissipation was also presented for the ternary system IM Aur in Borkovits et al. 2002).

In the present study we concentrate on a dynamically lessrelaxed scenario, namely when the inner binary has a significant eccentricity, i.e. the system is far from its synchronized and circularized state. Perhaps the most important feature of such systems is the apsidal motion effect (AME), i.e. the revolution of the orbital axis with a constant period which is determined mainly by the orbital separation, eccentricity, masses and the inner mass-distribution of the binary members. Nevertheless, several other physical processes also force AME. The two most significant ones are the perturbation of a third body, and the

Send offprint requests to: T. Borkovits relativistic apsidal motion. There is a small subgroup amongst these eccentric eclipsing binaries which have an additional importance, as their apsidal advance period is significantly (by more than $10-20 \%$ ) affected by the relativistic apsidal motion contribution. It is well-known, that the period of AME in these systems can be used as further confirmation or even as challenge for the General Relativity Theory. Unfortunately, these binaries necessarily have larger separation, so in such systems the apsidal motion period falls into the order of centuries or even of millenia. Consequently, in these systems first we have to solve the problem of the accurate determination of the apsidal period from a small portion of one revolution of the apsidal line, before we can label them as a challenge for the General Relativity Theory.

For our study we chose the eclipsing binary AS Camelopardalis, which is a member of an even smaller subgroup of the previously mentioned small group of the eccentric eclipsing binaries, as this system, together with approximately six others, shows a significantly lower apsidal motion rate than what is calculated from theory. Since this disrepancy was found for the first time at DI Herculis (Semeniuk 1968), several authors have investigated this phenomenon. A summary of their results can be found in Claret (1998). One of the possible ex- 
planations is the perturbations by a third component. The effect of the perturbations of a tertiary for the apsidal motion period has already been investigated only in a few previous studies Khodykin \& Vedeneyev 1997; Khodykin et al. 2004, and references therein). These papers mainly focused on the abovementioned two systems. Furthermore, in our opinion, these earlier studies have two fundamental disadvantages. First, the third body effect and the tidal effect were considered independently, and the resultant net apsidal motion period was calculated simply as an algebraic sum, which is very far from the reality, as it will be shown in the present paper. Second, the relation between the observed parameters and the physical quantities were not included in the scope of these papers. However, as we show, how the observed quantities, which are mainly deduced from some variants of the eclipsing $\mathrm{O}-\mathrm{C}$ curves, relate to the real apsidal motion period, is a sophisticated problem. Note, as we know, Claret (1998) was the first to mention this problem, nevertheless, in his paper this was not examined in the light of the perturbations by a third body.

In this paper we mainly focus on the short term observational consequences of the perturbations of a third body for an eccentric binary. We carry out both analytical and numerical studies. We give an analytical form of the time-dependence of the orbital elements of the close binary both in the dynamical and the observational reference frames up to fifth order in the eccentricity and related quantities. We also present the analytical form of the $\mathrm{O}-\mathrm{C}$ diagram of such a binary. We show that the complex variations of the orbital elements on a time-scale similar to the tidally forced apsidal motion period may result in significant discrepancies in the shape of the $\mathrm{O}-\mathrm{C}$ curve from the pure eccentric two body case, even without the remarkable real variation of the apsidal advance rate. Finally, we carry out some longer-time numerical integration to investigate the variation of the orbital as well as the stellar rotational parameters with and without dissipation.

It is important to note, that we restrict ourselves only for the simultaneous investigation of the third body and the tidally forced perturbations in the orbital elements in the frame of the classical, Newtonian mechanics. It may seem contradictory that despite the fact that our purpose is to give some acceptable explanations for the anomalously slow apsidal motion for those systems, where the relativistic contribution is remarkable, we do not consider the relativistic apsidal motion contribution at all. However, this contradiction can be resolved easily, as follows. The previous papers considered the three effects (tidal, third body, relativistic) as being independent. If we accept this, then our results should only be modified by some additive constants, which does not influence our qualitative results. Nevertheless, in the following we show that in the sense of the tidal and third body terms this is not the case. Similarly, we can assume, that neither the third body nor the relativistic terms can be considered independently from the tidal contribution. Consequently, to correctly consider the varying relativistic apsidal motion rate we would have to apply the relativistic formalism, which is far beyond the scope of this paper. However, in contrast to the tidal term, the relativistic apsidal motion has a notably smaller dependence on the eccentricity. This suggests that the linear, additive approximation is more realistic in this latter case. Consequently, we believe that our calculations give significant and well-applicable results.

We also omit the investigation of the effect of the nonsynchronized and even non-aligned stellar rotational axes on the perturbations of the orbital elements. Theoretically, it can be expected that such relatively young early-type eclipsing systems such as e.g. DI Her or AS Cam could have non-aligned rotational axes (cf.Zahn 1977), which can produce even a reversed net apsidal revolution. Analytical formulae are given e.g. in Shakura (1985), and Company et al. (1988). However, for the case of AS Cam in the thorough discussion Maloney et al. (1989) showed that this solution might be excluded, as the $v_{\text {rot }} \sin i$ values derived from radial velocity measurements of Hilditch (1972b) strongly suggest nearly synchronized rotation. Claret (1998) also refutes this solution in the case of DI Herculis. We also note that although in the presence of a third companion, stellar precession could be forced by the misalignment of the orbital planes even in the case when perfect synchronization is expected. A small amount of amplitude precession of the rotational axes actually occured in our numerical integrations presented in Sect. 3 Nevertheless, their amplitudes are so small that they could not affect the apsidal motion significantly

In the next section we give the general mathematical form of the orbital elements and the $\mathrm{O}-\mathrm{C}$ curve of an eccentric eclipsing binary when the revolution of the stars are affected by both tidal interactions and third-body perturbations. Then in Sect. 3 we present several short-time numerical integrations with different initial configurations of the AS Camelopardalis system for supporting the analytical results of Sect. 2, and, furthermore, we also study the dynamical evolution of the system on a longer time-scale, including also dissipative forces. In Sect. 4 we further discuss our results and conclude. Finally, in Appendix $\triangle$ we describe our mathematical calculations in details.

\section{Mathematical form of the O-C in a tidally and third-body perturbed eccenteric eclipsing binary}

In a previous paper (Borkovits et al. 2003) we calculated the effect of the third-body perturbations on the moments of the eclipsing minima of such eclipsing binaries which are members of close hierarchical triple stellar systems. Here we mainly follow the same method described that paper, so we give here only a brief summary, except the steps where we substantially modified the earlier methods.

\subsection{General considerations and equations of the problem}

As is well-known, at the moment of the mid-eclipse

$u \approx \pm \frac{\pi}{2}+2 k \pi$

where $u$ is the true longitude measured from the intersection of the orbital plane and the plane of sky, and $k$ is an integer. An exact equality stands only if the binary has a circular orbit, or if the orbit is seen edge-on exactly (for the correct inclination dependence of the occurrence of the mid-eclipses see Gimènez \& Garcia-Pelayo 1983). This latter condition is almost satisfied in those binaries which are of interest to us now. It is known from the textbooks of celestial mechanics, that

$$
\begin{aligned}
\dot{u} & =\frac{c}{\rho_{1}^{2}}-\dot{\Omega} \cos i, \\
& =\mu^{1 / 2} a^{-3 / 2}\left(1-e^{2}\right)^{-3 / 2}(1+e \cos v)^{2}-\dot{\Omega} \cos i,
\end{aligned}
$$

consequently, the moment of the $N$-th primary minimum after an epoch $t_{0}$ can be calculated as

$$
\int_{t_{0}}^{t_{N}} \mathrm{~d} t=\int_{\pi / 2}^{2 N \pi+\pi / 2} \frac{a^{3 / 2}}{\mu^{1 / 2}} \frac{\left(1-e^{2}\right)^{3 / 2}}{[1+e \cos (u-\omega)]^{2}} \frac{\mathrm{d} u}{1-\frac{\rho_{1}^{2}}{c_{1}} \dot{\Omega} \cos i}
$$




$$
\approx \int \frac{a^{3 / 2}}{\mu^{1 / 2}} \frac{\left(1-e^{2}\right)^{3 / 2}}{[1+e \cos (u-\omega)]^{2}}\left(1+\frac{\rho_{1}^{2}}{c_{1}} \dot{\Omega} \cos i\right) \mathrm{d} u .
$$

In the equations above $c_{1}$ denotes the specific angular momentum of the inner binary, $\rho_{1}$ is the radius vector of the secondary with respect to the primary, while the orbital elements have their usual meanings. Furthermore, in Eq. (3) we applied that the true anomaly can be written as $v=u-\omega$. Nevertheless, to avoid any confusion we emphasize that the angular elements (i.e. $u, \omega, \Omega$, i) are expressed in the "observational" frame of reference, that is, its fundamental plane is the plane of the sky, and $u$, as well as $\omega$ is measured from the intersection of the binary's orbital plane with that plane, while $\Omega$ is measured along the plane of the sky from an arbitrary origin. In order to evaluate Eq. (3) first we have to express the perturbations in the orbital elements with respect to $u$.

It is well known from the basic works of the three-body problem that in the present problem the perturbations in the orbital elements are effective on three different time-scales. Nevertheless, the so-called "short-term", as well as the "long-term" perturbations can be omitted due to their small amplitude. Strictly speaking, the second kind of the above perturbations might reach the limit of detectability in some systems (see Borkovits et al.2003), but from our point of view the "apse-node" terms have an exclusive importance. So, in what follows we concentrate on the so-called "apse-node" time-scale perturbative terms. They can be divided into two groups according to their different origin in Eq. (3). First, the "apse-node" time-scale perturbations in the orbital elements $e$ and $\omega$ arises also naturally in the formula above (as is well-known, there are neither "apse-node"-type, nor secular perturbations in the semi-major axis $a$ ). We will refer to this group in the following as indirect perturbations. Furthermore, some other terms which represent low-amplitude, short-period perturbations in $a, e, \omega$ give large-amplitude "apse-node" terms in $\dot{u}$ due to the multiplication with some of the $\cos n v$ terms. These are the direct perturbations in the orbital motion. Although our calculation of these latter direct perturbations would give back the first group too, we found that it is more convenient to calculate the two groups in two different ways.

First, we consider the indirect perturbations. As one can see later (e.g. Eqs. [84], 85]), the variation in both the eccentricity, and the argument of periastron during a few revolutions can be expressed as

$$
\begin{aligned}
\Delta e & \sim e\left(\frac{P}{P^{\prime}}\right)^{2} \Delta u, \\
\Delta \omega & \sim\left(\frac{P}{P^{\prime}}\right)^{2} \Delta u,
\end{aligned}
$$

so it is a quite good approximation to carry out the integration Eq. (3) first for one revolution treating $a, e$ and $\omega$ formally as constant. In this case taking into account only the first term on the right hand side (rhs) we arrive at an analogue of the wellknown Keplerian equation, which has the following closed solution

$$
\begin{gathered}
\bar{P}_{I}=\frac{P}{2 \pi}\left[2 \arctan \left(\sqrt{\frac{1-e}{1+e}} \frac{\cos \omega}{1+\sin \omega}\right)-\left(1-e^{2}\right)^{1 / 2} \frac{e \cos \omega}{1+e \sin \omega}\right], \\
\bar{P}_{I I}=\frac{P}{2 \pi}\left[2 \arctan \left(\sqrt{\frac{1-e}{1+e}} \frac{-\cos \omega}{1-\sin \omega}\right)+\left(1-e^{2}\right)^{1 / 2} \frac{e \cos \omega}{1-e \sin \omega}\right],
\end{gathered}
$$

for the two types of minima, respectively. (Here $P$ denotes the anomalistic or Keplerian period which is considered to be constant.) Note, that instead of the exact forms above, naturally its expansion is used widely (as in this paper), which is as follows, up to the fifth order in $e$ :

$$
\begin{aligned}
\bar{P}_{I, I I}= & P_{\mathrm{s}} E+\frac{P}{2 \pi}\left[ \pm \frac{1}{2} \pi \mp 2 e \cos \omega+\left(\frac{3}{4} e^{2}+\frac{1}{8} e^{4}\right) \sin 2 \omega\right. \\
& \left. \pm\left(\frac{1}{3} e^{3}+\frac{1}{8} e^{5}\right) \cos 3 \omega-\frac{5}{32} e^{4} \sin 4 \omega \mp \frac{3}{40} e^{5} \cos 5 \omega\right],
\end{aligned}
$$

where $P_{\mathrm{s}}$ is the sidereal (or eclipsing) period of, for example, the first cycle, and $E$ is the cycle-number. Nevertheless, the difference of the two quantities (usually denoted by $D$ ) is often quoted in the literature in its closed form.

We now formulate the direct perturbations. To do this we write $e \cos v$ as

$$
\begin{aligned}
e \cos v= & e \cos \omega \cos u+e \sin \omega \sin u \\
= & (e \cos \omega)_{0} \cos u+(e \sin \omega)_{0} \sin u+ \\
& +\int_{u_{0}}^{u}\left(\frac{\mathrm{d} e}{\mathrm{~d} u^{\prime}} \cos \omega-e \frac{\mathrm{d} \omega}{\mathrm{d} u^{\prime}} \sin \omega\right) \mathrm{d} u^{\prime} \cos u+ \\
& +\int_{u_{0}}^{u}\left(\frac{\mathrm{d} e}{\mathrm{~d} u^{\prime}} \sin \omega+e \frac{\mathrm{d} \omega}{\mathrm{d} u^{\prime}} \cos \omega\right) \mathrm{d} u^{\prime} \sin u
\end{aligned}
$$

When we concentrate only on the short-period terms in the derivatives (i.e. those which are functions of $\cos n u$ or $\sin n u$ ), we can take the $\cos \omega$ and $\sin \omega$ terms out of the integrand, and carry out the integrations only for the derivatives, so the "apsenode" time-scale direct perturbations in $e \cos v$ could be derived from

$e \cos v_{\mathrm{dir}}=\cos v \int_{u_{0}}^{u}\left(\frac{\mathrm{d} e}{\mathrm{~d} u^{\prime}}\right)_{\mathrm{u}} \mathrm{d} u^{\prime}-\sin v \int_{u_{0}}^{u}\left(e \frac{\mathrm{d} \omega}{\mathrm{d} u^{\prime}}\right)_{\mathrm{u}} \mathrm{d} u^{\prime}$,

where the subscript ${ }_{\mathrm{u}}$ refers to those terms which contain $\pm u$ in their arguments, and, generally

$$
\begin{aligned}
e^{m} \cos n v_{\mathrm{dir}}= & m e^{m-1} \cos n v \int_{u_{0}}^{u}\left(\frac{\mathrm{d} e}{\mathrm{~d} u^{\prime}}\right)_{\mathrm{nu}} \mathrm{d} u^{\prime}- \\
& -n e^{m-1} \sin n v \int_{u_{0}}^{u}\left(e \frac{\mathrm{d} \omega}{\mathrm{d} u^{\prime}}\right)_{\mathrm{nu}} \mathrm{d} u^{\prime} .
\end{aligned}
$$

Furthermore, the direct perturbations coming from the semimajor axis can be calculated as

$$
(\dot{u})_{\mathrm{a}-\mathrm{dir}}^{-1}=\frac{3}{2} \frac{1}{a} \frac{\mu^{-1 / 2} a^{3 / 2}\left(1-e^{2}\right)^{3 / 2}}{(1+e \cos v)^{2}} \int \frac{\mathrm{d} a}{\mathrm{~d} u^{\prime}} \mathrm{d} u^{\prime} .
$$

The derivatives are as follows:

$$
\begin{aligned}
\frac{\mathrm{d} a}{\mathrm{~d} u} & =\frac{2 a^{3 / 2}}{\sqrt{\mu\left(1-e^{2}\right)}}\left[f_{\mathrm{r}} e \sin v+f_{\mathrm{t}}(1+e \cos v)\right] \frac{\mathrm{d} t}{\mathrm{~d} u} \\
& \approx \frac{2 a^{3}}{\mu} \frac{1-e^{2}}{(1+e \cos v)^{2}}\left[f_{\mathrm{r}} e \sin v+f_{\mathrm{t}}(1+e \cos v)\right] \\
\frac{\mathrm{d} e}{\mathrm{~d} u} & \approx \frac{a^{2}}{\mu} \frac{\left(1-e^{2}\right)^{2}}{\left(1+e \cos v^{2}\right.}\left[f_{\mathrm{r}} \sin v+f_{\mathrm{t}}\left(\cos v+\frac{\cos v+e}{1+e \cos v}\right)\right] \\
e \frac{\mathrm{d} \omega}{\mathrm{d} u} & \approx \frac{a^{2}}{\mu} \frac{\left(1-e^{2}\right)^{2}}{\left(1+e \cos v^{2}\right.}\left[-f_{\mathrm{r}} \cos v+f_{\mathrm{t}}\left(\sin v+\frac{\sin v}{1+e \cos v}\right)-\right. \\
\frac{\mathrm{d} \Omega}{\mathrm{d} u} & \approx \frac{\left.a^{2} \frac{e \cot i \sin u}{1+e \cos v}\right]}{\mu} \frac{\left(1-e^{2}\right)^{2}}{(1+e \cos v)^{2}} f_{\mathrm{n}} \frac{e \sin u}{\sin i(1+e \cos v)}
\end{aligned}
$$


where $f_{\mathrm{r}, \mathrm{t}, \mathrm{n}}$ represent the radial, transversal and normal components of the perturbing force (see later). We applied the following approximation:

$$
\frac{\mathrm{d} t}{\mathrm{~d} u} \approx \frac{\rho_{1}^{2}}{c_{1}} \text {. }
$$

Finally, considering the last term on the rhs of Eq. (3), by the use of Eqs. (15) and (16) it can be seen that all the direct "apsenode" terms in $e^{m} \cos n v$-s which would occur from the $f_{\mathrm{n}}$ forcecomponent are cancelled by the opposite-sign term in $\frac{\rho_{1}^{2}}{c_{1}} \frac{\mathrm{d} \Omega}{\mathrm{d} u} \cos i$, and only the indirect term from $\mu^{-1 / 2} a^{3 / 2} \frac{\mathrm{d} \Omega}{\mathrm{d} u} \cos i$ gives a further contribution.

As a next step we calculate the secular perturbations in the above-listed orbital elements. For the calculations we truncated the perturbing force at the second order term. In this case the force components effective on the close binary are as follows:

$$
\begin{aligned}
f_{\mathrm{r} 1}= & \frac{3}{8} \frac{G m_{3}}{\rho_{2}^{2}} \frac{\rho_{1}}{\rho_{2}}\left[(1+I)^{2} \cos \left(2 u^{\prime}-2 u-\alpha\right)\right. \\
& \left.+(1-I)^{2} \cos \left(2 u^{\prime}+2 u-\beta\right)+2\left(1-I^{2}\right) \cos \left(2 u-2 u_{\mathrm{m}}\right)\right], \\
f_{\mathrm{r} 2}= & \frac{3}{4} \frac{G m_{3}}{\rho_{2}^{2}} \frac{\rho_{1}}{\rho_{2}}\left[\left(1-I^{2}\right) \cos \left(2 u^{\prime}-2 u_{\mathrm{m}}^{\prime}\right)+I^{2}-\frac{1}{3}\right] \\
f_{\mathrm{r} 3}= & -\frac{\mu}{\rho_{1}^{4}}\left(\frac{\mathcal{T}_{2}}{\rho_{1}^{3}}+\mathcal{R}\right) \\
f_{\mathrm{t}}= & \frac{3}{8} \frac{G m_{3}}{\rho_{2}^{2}} \frac{\rho_{1}}{\rho_{2}}\left[(1+I)^{2} \sin \left(2 u^{\prime}-2 u-\alpha\right)\right. \\
& \left.-(1-I)^{2} \sin \left(2 u^{\prime}+2 u-\beta\right)-2\left(1-I^{2}\right) \sin \left(2 u-2 u_{\mathrm{m}}\right)\right], \\
f_{\mathrm{n}}= & -\frac{3}{4} \frac{G m_{3}}{\rho_{2}^{2}} \frac{\rho_{1}}{\rho_{2}}\left\{2 \cos \left(u-u_{\mathrm{m}}\right) \sin 2\left(u^{\prime}-u_{\mathrm{m}}^{\prime}\right) \sin i_{\mathrm{m}}+\right. \\
& {\left.\left[1-\cos 2\left(u^{\prime}-u_{\mathrm{m}}^{\prime}\right)\right] \sin \left(u-u_{\mathrm{m}}\right) \sin 2 i_{\mathrm{m}}\right\} }
\end{aligned}
$$

We divided the radial component into three parts, relative to their different significances. Namely, $f_{\mathrm{r} 3}$ is the tidal term, while amongst the two three-body terms, $f_{\mathrm{r} 2}$ is formally analogous to $f_{\mathrm{r} 3}$, i.e. gives a similar secular contribution to the considered perturbations (see later). Furthermore, $I$ stands for the cosine of the mutual inclination $\left(i_{\mathrm{m}}\right)$ of the two orbits, $\rho_{2}$ is the radius vector of the tertiary, $u, u^{\prime}$ are the true longitude of the close and the wide orbits measured from the plane of the sky, while $u_{\mathrm{m}}, u_{\mathrm{m}}^{\prime}$ are the true longitudes of the intersection of the orbital planes measured from the plane of the sky along the close and the wide orbital planes, respectively (see Fig. (1). Finally, the contributions of the mutual tidal, and the rotational oblateness are as follows:

$$
\begin{gathered}
\mathcal{T}_{2}=6\left(\frac{m_{2}}{m_{1}} k_{2}^{(1)} R_{1}^{5}+\frac{m_{1}}{m_{2}} k_{2}^{(2)} R_{2}^{5}\right), \\
\mathcal{R}=\frac{k_{2}^{(1)} R_{1}^{5} \omega_{z_{1}^{\prime}}^{2}}{G m_{1}}+\frac{k_{2}^{(2)} R_{2}^{5} \omega_{z_{2}^{\prime}}^{2}}{G m_{2}},
\end{gathered}
$$

where $k_{2}^{(1,2)}$ are the usual apsidal motion constants, $R_{1,2}$ are the average radii of the stars, while $\omega_{z_{1,2}^{\prime}}$ are the rotational angular velocities of the star (which are treated as constant here).

As in the present approximation there are no "apse-node" or secular changes in the orbital elements of the tertiary, we considered its orbital elements as constant. Although the equations of perturbations can be written directly for the above-mentioned orbital elements, it is more convenient to use the equations for the orbital elements expressed in the so-called dynamical frame of

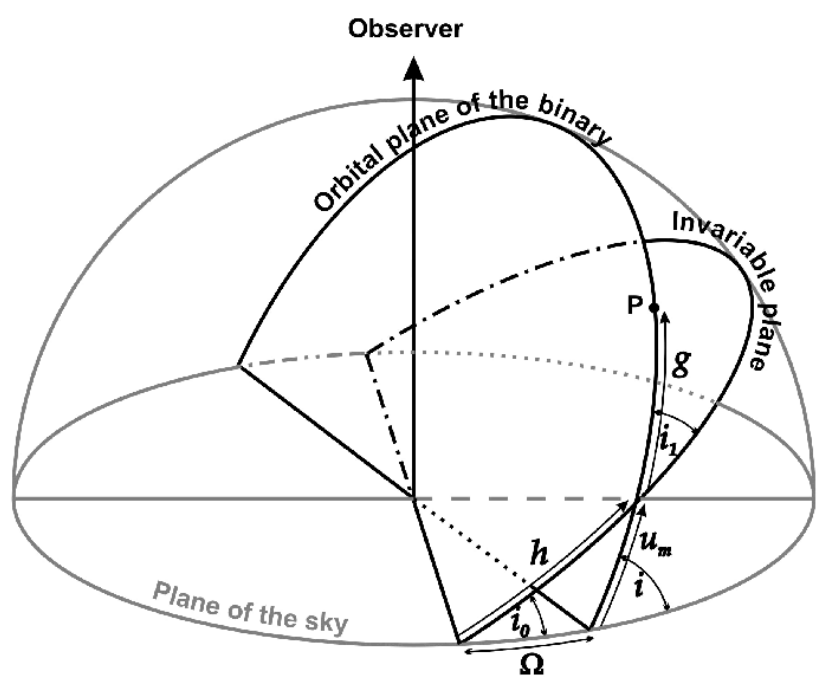

Fig. 1. The spatial configuration of the system.

reference, in which the fundamental plane is the invariable plane of the triple system. The angular orbital elements in the observational frame can then be expressed from these by the formulae of spherical geometry. To avoid any confusion, the argument of periastron, and the longitude of the corresponding ascending node in this system are denoted by $g$ and $h$, respectively (which are their usual notations in the perturbation theories). Furthermore, the inclination of the orbital plane of the binary with respect to the invariable plane is denoted by $i_{1}$. It can be clearly seen, that the relation between the two periastron elements are $\omega=g+u_{\mathrm{m}}$ (see Fig. 11). 1 In the frame of our approximation the studied problem is reduced to one degree of freedom. Consequently, first we express the variations of all the interesting orbital elements in the function of the argument of periastron $g$ of the binary in the dynamical reference frame, and then, we give the $g(u)$ function. So, the secular parts of the perturbational equations are as follows:

$$
\begin{aligned}
\frac{\mathrm{d} u}{\mathrm{~d} g} & =\frac{1-\frac{\rho_{1}^{2}}{c_{1}} \dot{\Omega} \cos i}{A+B \cos 2 g}, \\
& \approx \frac{1}{A+B \cos 2 g}-\frac{\mathrm{d} \Omega}{\mathrm{d} g} \cos i, \\
\frac{\mathrm{d} e}{\mathrm{~d} g} & =\frac{e A_{\mathrm{t}} \sin 2 g}{A+B \cos 2 g}, \\
\frac{\mathrm{d} h}{\mathrm{~d} g} & =-\frac{1}{\cos i_{1}} \frac{A_{\mathrm{n} 1}+A_{\mathrm{n} 2}-A_{\mathrm{n} 2} \cos 2 g}{A+B \cos 2 g}, \\
\frac{\mathrm{d} i_{1}}{\mathrm{~d} g} & =\tan i_{1} \frac{A_{\mathrm{n} 2} \sin 2 g}{A+B \cos 2 g}, \\
& =-\frac{e}{1-e^{2}} \cot i_{\mathrm{m}} \frac{\mathrm{d} e}{\mathrm{~d} g}, \\
\frac{\mathrm{d} \delta}{\mathrm{d} g} & =\frac{A_{\mathrm{d}}+B_{\mathrm{d}} \cos 2 g}{A+B \cos 2 g},
\end{aligned}
$$

1 Note, that in some studies on apsidal motion for the equivalents of Eq. 6) the sign $\varpi$ is used, which is meant as $g+h$. Naturally, it is correct if the perturbative force lies perfectly in the orbital plane of the binary, which is fulfilled as far as only the tidal effect of the distorted binary members with perpendicular rotation axes are considered, nevertheless, in the present situation this is no longer the case. 
where $\delta$ denotes the direct perturbations in $(\dot{u})^{-1}$, while for the angular elements in the observational frame of reference we obtain that

$$
\begin{aligned}
\frac{\mathrm{d} \Omega}{\mathrm{d} g}= & \frac{\mathrm{d} h}{\mathrm{~d} g} \frac{\cos I_{0}-\cos i_{1} \cos i}{1-\cos ^{2} i}+\frac{\mathrm{d} i_{1}}{\mathrm{~d} g} \frac{\sin I_{0} \sin h}{1-\cos ^{2} i}, \\
\frac{\mathrm{d} \omega}{\mathrm{d} g}= & 1+\frac{\mathrm{d} u_{\mathrm{m}}}{\mathrm{d} g} \\
= & 1+\frac{\mathrm{d} h}{\mathrm{~d} g} \cos i_{1}-\frac{\mathrm{d} \Omega}{\mathrm{d} g} \cos i \\
= & 1+\frac{\mathrm{d} h}{\mathrm{~d} g} \frac{\cos i_{1}-\cos I_{0} \cos i}{1-\cos ^{2} i}-\frac{\mathrm{d} i_{1}}{\mathrm{~d} g} \frac{\sin I_{0} \cos i \sin h}{1-\cos ^{2} i} \\
= & \frac{A-A_{\mathrm{n} 1}-A_{\mathrm{n} 2}+A_{\mathrm{t}} \cos 2 g}{A+B \cos 2 g}-\frac{\mathrm{d} \Omega}{\mathrm{d} g} \cos i, \\
\frac{\mathrm{d} i}{\mathrm{~d} g}= & -\frac{\mathrm{d} h}{\mathrm{~d} g} \frac{\sin I_{0} \sin i_{1} \sin h}{\sin i} \\
& +\frac{\mathrm{d} i_{1}}{\mathrm{~d} g} \frac{\cos I_{0} \sin i_{1}+\cos i_{1} \sin I_{0} \cos h}{\sin i} .
\end{aligned}
$$

In the equations above the tidal contributions are:

$$
\begin{aligned}
A_{\mathrm{r} 3}= & \frac{1}{2 a^{5}}\left[5 \mathcal{T}_{2} \frac{1+\frac{3}{2} e^{2}+\frac{1}{8} e^{4}}{\left(1-e^{2}\right)^{5}}+2 \mathcal{R} \frac{1}{\left(1-e^{2}\right)^{2}}\right], \\
A_{\mathrm{dr} 3}= & -\frac{2}{a^{5}}\left[\mathcal{T}_{2} \frac{1+\frac{37}{8} e^{2}+\frac{59}{16} e^{4}+\frac{113}{32} e^{6}}{\left(1-e^{2}\right)^{5}}+\right. \\
& \left.+\mathcal{R} \frac{1+\frac{5}{4} e^{2}+\frac{5}{4} e^{4}+\frac{5}{4} e^{6}}{\left(1-e^{2}\right)^{2}}\right],
\end{aligned}
$$

while the third body terms are as follows:

$$
\begin{aligned}
A_{\mathrm{G}} & =\frac{15}{8} \frac{m_{3}}{m_{123}}\left(\frac{P}{P^{\prime}}\right)^{2}\left(1-e^{2}\right)^{-3 / 2}, \\
A_{\mathrm{r} 2} & =\frac{3}{5} A_{\mathrm{G}}\left(1-e^{2}\right)^{1 / 2}\left(I^{2}-\frac{1}{3}\right), \\
A_{\mathrm{dr} 2} & =\frac{4}{3}\left(1+\frac{25}{8} e^{2}+\frac{15}{8} e^{4}+\frac{95}{64} e^{6}\right) A_{\mathrm{r} 2}, \\
A_{\mathrm{t}} & =A_{\mathrm{G}}\left(1-e^{2}\right)^{1 / 2}\left(1-I^{2}\right), \\
A_{\mathrm{dt}} & =\frac{51}{20} e^{2}\left(1+\frac{31}{51} e^{2}+\frac{23}{48} e^{4}\right) A_{\mathrm{t}}, \\
A_{\mathrm{n} 1} & =\frac{2}{5} A_{\mathrm{G}}\left(1-e^{2}\right)^{1 / 2}\left[I^{2}+\frac{C_{1}}{C_{2}} I\right], \\
A_{\mathrm{n} 2} & =A_{\mathrm{G}} \frac{e^{2}}{\left(1-e^{2}\right)^{1 / 2}}\left[I^{2}+\frac{C_{1}}{C_{2}} I\right],
\end{aligned}
$$

and, finally,

$$
\begin{aligned}
A= & A_{\mathrm{r} 3}+A_{\mathrm{r} 2}+A_{\mathrm{n} 1}+A_{\mathrm{n}_{2}} \\
= & \frac{1}{2 a^{5}}\left[5 \mathcal{T}_{2} \frac{1+\frac{3}{2} e^{2}+\frac{1}{8} e^{4}}{\left(1-e^{2}\right)^{5}}+2 \mathcal{R} \frac{1}{\left(1-e^{2}\right)^{2}}\right] \\
& +A_{\mathrm{G}}\left[\frac{1}{\left(1-e^{2}\right)^{1 / 2}} I^{2}-\frac{1}{5}\left(1-e^{2}\right)^{1 / 2}+\frac{2}{5} \frac{1+\frac{3}{2} e^{2}}{\left(1-e^{2}\right)^{1 / 2}} \frac{C_{1}}{C_{2}} I\right], \\
B= & A_{\mathrm{t}}-A_{\mathrm{n} 2} \\
= & A_{\mathrm{G}}\left[\left(1-e^{2}\right)^{1 / 2}-\frac{1}{\left(1-e^{2}\right)^{1 / 2}} I^{2}-\frac{e^{2}}{\left(1-e^{2}\right)^{1 / 2}} \frac{C_{1}}{C_{2}} I\right], \\
A_{\mathrm{d}}= & A_{\mathrm{dr} 3}+A_{\mathrm{dr} 2}, \\
B_{\mathrm{d}}= & A_{\mathrm{dt}},
\end{aligned}
$$

At the calculation of the formulae above it was also used that

$$
\begin{aligned}
& \cos i_{1}=\frac{\boldsymbol{C} \boldsymbol{C}_{1}}{C C_{1}}, \\
& \sin i_{1}=\frac{\left|\boldsymbol{C} \times \boldsymbol{C}_{1}\right|}{C C_{1}},
\end{aligned}
$$

where $\boldsymbol{C}_{1}$ means the orbital angular momentum of the close binary, while $\boldsymbol{C}$ is the same for the whole system. Supposing that the rotational angular momenta of the three stars are negligible we obtain that

$$
\begin{aligned}
& \cos i_{1}=\frac{C_{1}}{C}+\frac{C_{2}}{C} I, \\
& \sin i_{1}=-\frac{C_{2}}{C} \sin i_{\mathrm{m}},
\end{aligned}
$$

and it is well known, that

$$
\begin{aligned}
& C_{1}=\frac{m_{1} m_{2}}{m_{12}} \sqrt{G m_{12} a\left(1-e^{2}\right)}, \\
& C_{2}=\frac{m_{12} m_{2}}{m_{123}} \sqrt{G m_{123} a^{\prime}\left(1-e^{\prime 2}\right)} .
\end{aligned}
$$

\subsection{Solution for edge-on binary orbits with small eccentricity variations}

\subsubsection{Closed form solutions for the binary's orbital elements}

For the first time we assume an edge-on binary orbit in the observational frame, which is a plausible expectation in the case of the relatively wider eccentric eclipsing binaries. Consequently, at this stage we omit terms multiplied by $\cos i$. We consider Eqs. (25)-(32). One can see that if $B \geq A$ these equations become singular at certain directions of the axis. This is exactly the case which defines the so-called Kozai resonance (Kozai 1962). We are interested in such binaries where $A>B$, i.e. this resonance does not occur. In this case as far as the coefficients at the rhs of the equations can be treated as constant, or at least their variations are small, all the equations have closed solution, which, for $B \neq 0$ are as follows:

$$
\begin{aligned}
u= & u_{0}+\frac{1}{A} \frac{1}{\sqrt{1-E^{2}}} \arctan \left(\sqrt{\frac{1-E}{1+E}} \tan g\right)_{g_{0}}^{g}, \\
e= & e_{0}-\frac{1}{2} e \frac{A_{\mathrm{t}}}{B} \ln \left(\frac{1+E \cos 2 g}{1+E \cos 2 g_{0}}\right), \\
h= & h_{0}+\frac{1}{\cos i_{1}} \frac{A_{\mathrm{n} 2}}{B}\left(g-g_{0}\right)-\frac{1}{\cos i_{1}}\left(\frac{A_{\mathrm{n} 1}+A_{\mathrm{n} 2}}{A}+\frac{A_{\mathrm{n} 2}}{B}\right) \\
& \times \frac{1}{\sqrt{1-E^{2}}} \arctan \left(\sqrt{\frac{1-E}{1+E}} \tan g\right)_{g_{0}}^{g}, \\
i_{1}= & \left(i_{1}\right)_{0}-\frac{1}{2} \tan i_{1} \frac{A_{\mathrm{n} 2}}{B} \ln \left(\frac{1+E \cos 2 g}{1+E \cos 2 g_{0}}\right), \\
\omega= & \omega_{0}+\frac{A_{\mathrm{t}}}{B}\left(g-g_{0}\right)-\left(\frac{A_{\mathrm{n} 1}+A_{\mathrm{n} 2}}{A}+\frac{A_{\mathrm{n} 2}}{B}\right) \\
& \times \frac{1}{\sqrt{1-E^{2}}} \arctan \left(\sqrt{\frac{1-E}{1+E}} \tan g\right)_{g}^{g}, \\
\delta= & \delta_{0}+\frac{B_{\mathrm{d}}}{B}\left(g-g_{0}\right) \\
& +\left(\frac{A_{\mathrm{d}}}{A}-\frac{B_{\mathrm{d}}}{B}\right) \frac{1}{\sqrt{1-E^{2}}} \arctan \left(\sqrt{\frac{1-E}{1+E}} \tan g\right)_{g_{0}}^{g},
\end{aligned}
$$


where

$E=\frac{B}{A}$.

Eqs. (53) and (55) are equivalent to the results of Söderhielm (1984). Nevertheless, in his paper the eccentricity equation was not calculated, as the eccentricity was considered as strictly constant. Furthermore, we stress again, that the last equation for $\omega$ is calculated in the observational and not in the dynamical frame of reference. The first equation reveals that in this case we get the following constant angular velocity (in $P_{\mathrm{s}}$ units) for the apsidal motion in the dynamical system:

$\Pi=A \sqrt{1-E^{2}}$.

Similarly, the secular terms, i.e. the mean angular velocities of the invariant node $(h)$, and the observable argument of periastron $(\omega)$ are as follows:

$$
\begin{aligned}
\mathcal{H}_{0} & =\frac{1}{\cos i_{1}}\left[\frac{A_{\mathrm{n} 2}}{B}-\left(\frac{A_{\mathrm{n} 1}+A_{\mathrm{n} 2}}{A}+\frac{A_{\mathrm{n} 2}}{B}\right) \frac{1}{\sqrt{1-E^{2}}}\right] \Pi, \\
\mathcal{O}_{0} & =\Pi+\cos i_{1} \mathcal{H}_{0} \\
& =\left[\frac{A_{\mathrm{t}}}{B}-\left(\frac{A_{\mathrm{n} 1}+A_{\mathrm{n} 2}}{A}+\frac{A_{\mathrm{n} 2}}{B}\right) \frac{1}{\sqrt{1-E^{2}}}\right] \Pi,
\end{aligned}
$$

while the secular part of the direct perturbations gives

$\mathcal{D}_{0}=\left[\frac{B_{\mathrm{d}}}{B}+\left(\frac{A_{\mathrm{d}}}{A}+\frac{B_{\mathrm{d}}}{B}\right) \frac{1}{\sqrt{1-E^{2}}}\right] \Pi$.

In the following we introduce the new variable $\mathcal{G}$ as

$\mathcal{G}=\arctan \left(\sqrt{\frac{1-E_{0}}{1+E_{0}}} \tan g\right)$.

It can be seen easily that

$\frac{\mathrm{d} u}{\mathrm{~d} g} \frac{\mathrm{d} g}{\mathrm{~d} \mathcal{G}}=\frac{1}{A} \frac{1-E_{0} \cos 2 \mathcal{G}}{1-E E_{0}+\left(E-E_{0}\right) \cos 2 \mathcal{G}} \frac{\sqrt{1-E_{0}^{2}}}{1-E_{0} \cos 2 \mathcal{G}}$,

and, as in the current approximation $E=E_{0}=$ const.,

$u-u_{0}=\Pi^{-1}\left(\mathcal{G}-\mathcal{G}_{0}\right)$.

Similarly,

$$
\begin{aligned}
e= & e_{0}\left(1+\epsilon_{0}\right)+\frac{1}{2} e_{0}\left(\frac{A_{\mathrm{t}}}{B}\right)_{0} \ln \left(1-E_{0} \cos 2 \mathcal{G}\right), \\
g= & \arctan \left(\sqrt{\frac{1+E_{0}}{1-E_{0}}} \tan \mathcal{G}\right) \\
h= & h_{0}^{*}-\frac{1}{\cos i_{1}}\left(A_{\mathrm{n} 1}+A_{\mathrm{n} 2}+\frac{A_{\mathrm{n} 2}}{E}\right)_{0} \frac{1}{\Pi_{0}} \mathcal{G} \\
& +\frac{1}{\cos i_{1}}\left(\frac{A_{\mathrm{n} 2}}{B}\right)_{0} \arctan \left(\sqrt{\frac{1+E_{0}}{1-E_{0}}} \tan \mathcal{G}\right), \\
i_{1}= & \left(i_{1}\right)_{0}^{*}+\frac{1}{2} \tan i_{1}\left(\frac{A_{\mathrm{n}}}{B}\right)_{0} \ln \left(1-E_{0} \cos 2 \mathcal{G}\right), \\
\omega= & \omega_{0}^{*}-\left(A_{\mathrm{n} 1}+A_{\mathrm{n} 2}+\frac{A_{\mathrm{n} 2}}{E}\right)_{0} \frac{1}{\Pi_{0}} \mathcal{G} \\
& +\left(\frac{A_{\mathrm{t}}}{B}\right)_{0} \arctan \left(\sqrt{\frac{1+E_{0}}{1-E_{0}}} \tan \mathcal{G}\right), \\
\delta= & \delta_{0}^{*}+\left(A_{\mathrm{d}}-\frac{B_{\mathrm{d}}}{E}\right)_{0} \frac{1}{\Pi_{0}} \mathcal{G} \\
& +\left(\frac{B_{\mathrm{d}}}{B}\right)_{0} \arctan \left(\sqrt{\frac{1+E_{0}}{1-E_{0}}} \tan \mathcal{G}\right),
\end{aligned}
$$

where

$$
\begin{aligned}
\epsilon_{0}= & -\frac{1}{2}\left(\frac{A_{\mathrm{t}}}{B}\right)_{0} \ln \left(1-E_{0} \cos 2 \mathcal{G}_{0}\right), \\
h_{0}^{*}= & h_{0}-\frac{1}{\cos i_{1}}\left(\frac{A_{\mathrm{n} 2}}{B}\right)_{0} g_{0} \\
& +\left(A_{\mathrm{n} 1}+A_{\mathrm{n} 2}+\frac{A_{\mathrm{n} 2}}{E}\right)_{0} \frac{1}{\Pi_{0}} \mathcal{G}_{0}, \\
\left(i_{1}\right)_{0}^{*}= & \left(i_{1}\right)_{0}-\frac{1}{2}\left(\tan i_{1} \frac{A_{\mathrm{n}}}{B}\right)_{0} \ln \left(1-E_{0} \cos 2 \mathcal{G}_{0}\right), \\
\omega_{0}^{*}= & \omega_{0}-\left(\frac{A_{\mathrm{t}}}{B}\right)_{0} g_{0}+\left(A_{\mathrm{n} 1}+A_{\mathrm{n} 2}+\frac{A_{\mathrm{n} 2}}{E}\right)_{0} \frac{1}{\Pi_{0}} \mathcal{G}_{0} \\
= & \left(u_{\mathrm{m}}\right)_{0}+\left(h_{0}^{*}-h_{0}\right) \cos i_{1}, \\
\delta_{0}^{*}= & \delta_{0}-\left(\frac{B_{\mathrm{d}}}{B}\right)_{0} g_{0}-\left(A_{\mathrm{d}}-\frac{B_{\mathrm{d}}}{E}\right)_{0} \frac{1}{\Pi_{0}} \mathcal{G}_{0},
\end{aligned}
$$

respectively. In what follows, we omit the subscript ${ }_{0}$ from the parameters $E, \mathcal{E}$, because these parameters will always be used as constants, with the value calculated at $e=e_{0}$.

\subsubsection{The analytical form of the apsidal part of the $\mathrm{O}-\mathrm{C}$}

By the use of the Taylorian expansion of Eqs. (67), (71) and (72) we obtain for the analytical form of the apsidal part of the $\mathrm{O}-\mathrm{C}$ as follows:

$$
\begin{aligned}
& \frac{2 \pi}{P} \bar{P}=j \frac{1}{2} \pi-2 j e_{0}\left(1+\epsilon_{0}\right) \cos \left[\omega_{0}^{*}+(1+\mathcal{U}) \mathcal{G}\right] \\
& +\frac{3}{4} e_{0}^{2}\left(1+\epsilon_{0}\right)^{2} \sin \left[2 \omega_{0}^{*}+(2+2 \mathcal{U}) \mathcal{G}\right] \\
& +j \frac{1}{3} e_{0}^{3}\left(1+\epsilon_{0}\right)^{3} \cos \left[3 \omega_{0}^{*}+(3+3 \mathcal{U}) \mathcal{G}\right] \\
& +j e_{0}\left[\frac{1}{8}\left(1+\epsilon_{0}\right) \mathcal{E}^{2}+\frac{1}{4} \mathcal{E} E\right] \cos \left[\omega_{0}^{*}+(1+\mathcal{U}) \mathcal{G}\right] \\
& +j e_{0} \frac{1}{2} \epsilon_{0} \mathcal{E} \cos \left[\omega_{0}^{*}+(3+\mathcal{U}) \mathcal{G}\right] \\
& +j e_{0}\left[\mathcal{E}+\frac{1}{2} \epsilon_{0} \mathcal{E}\right] \cos \left[\omega_{0}^{*}-(1-\mathcal{U}) \mathcal{G}\right] \\
& +j e_{0}\left[\frac{1}{16}\left(1-\epsilon_{0}\right) \mathcal{E}^{2}-\frac{1}{8} \epsilon_{0} \mathcal{E} E\right] \cos \left[\omega_{0}^{*}+(5+\mathcal{U}) \mathcal{G}\right] \\
& +j e_{0}\left[-\frac{1}{16}\left(3+\epsilon_{0}\right) \mathcal{E}^{2}\right. \\
& \left.+\frac{1}{8}\left(2+\epsilon_{0}\right) \mathcal{E} E\right] \cos \left[\omega_{0}^{*}-(3-\mathcal{U}) \mathcal{G}\right] \\
& \left.+e_{0}^{2}\left[\frac{3}{8} \epsilon_{0}\left(1+\epsilon_{0}\right) \mathcal{E}\right] \sin \left[2 \omega_{0}^{*}+(4+2 \mathcal{U}) \mathcal{G}\right)\right] \\
& +e_{0}^{2}\left[-\frac{3}{8}\left(2+3 \epsilon_{0}+\epsilon_{0}^{2}\right) \mathcal{E}\right] \sin \left(2 \omega_{0}^{*}+2 \mathcal{U G}\right) \\
& +\frac{51}{40} e_{0}^{2} \mathcal{E} \sin 2 \mathcal{G}+O\left[(e, E)^{4}\right] .
\end{aligned}
$$

From now on we suppose, that $O(e)=O(E)=O(\mathcal{E})$, or more generally, $O(e)=O\left(\frac{A_{\mathrm{G}}}{A}\right)$. Furthermore,

$$
\begin{aligned}
\mathcal{E} & =\frac{A_{\mathrm{t}}}{A} \\
\mathcal{U} & =\frac{A_{\mathrm{n} 2}}{B}-\left(\frac{A_{\mathrm{n} 1}+A_{\mathrm{n} 2}}{A}+\frac{A_{\mathrm{n} 2}}{B}\right) \frac{1}{\sqrt{1-E^{2}}}
\end{aligned}
$$


the latter is the period ratio of $h \cos i_{1}$ (or in the present approximation, $u_{\mathrm{m}}$ ) and $g$. This term gives the relative difference of the speed of the apsidal advance in the observational and the dynamical frames of reference. Furthermore, note, that in this approximation the direct terms give only a small contribution to the $\mathrm{O}-\mathrm{C}$ (the last term of Eq. [78]). Nevertheless, the secular part of such perturbations are added to the observed eclipsing period. Finally, $j \pm 1$ for the two different types of minima.

We consider the two extreme cases. First, if the two orbits are coplanar (i.e. $I^{2}=1$ ), then the angular velocity of the apsidal motion is

$$
(1+\mathcal{U}) \Pi=A_{\mathrm{r} 2}+A_{\mathrm{r} 3} .
$$

Furthermore, as $\mathcal{E}=0$, (i.e. $\Delta e=0$, which is true as far as we do not consider the octuple term in the perturbing force), Eq. (78) reduces for its usual form, apart from the different period given by Eq. (81). Second, in the case of two perpendicular orbits (i.e. $I=0), \mathcal{U}$ diminishes, as well as $\mathcal{E}=E$ occurs, and, consequently, Eq. (78) also becomes somewhat simpler. Moreover, the more important feature is that these are generally the only two cases, when the $\mathrm{O}-\mathrm{C}$ curve has only one fundamental period. Finally, in these two extreme cases

$\dot{\Omega}=0$,

so the results above are rigorously correct for not only edge-on visible orbits, as far as the orbital eccentricity can be considered as constant on the rhs of the perturbation equations (25)-(33).

\subsection{Solution for the general case}

\subsubsection{Formulae for $e, g$ and $h$ - results and discussion on the dynamical apsidal motion period and on the nodal regression}

One can see that the assumption of the constant eccentricity remains plausible only if $B<<A$, or if $A_{\mathrm{t}} \approx 0$, which may happen in two different ways. The trivial case, when no third body exists in the system, or at least its influence can be disregarded with respect to the tidal forces, or the other possibility is, that the two orbits are nearly coplanar. (Note, that this latter case usually also could satisfy the $B<<A$ condition, as in this case $B$ is in the order of $e^{2} A_{\mathrm{G}}$.) Nevertheless, in the really interesting systems neither of these conditions are fulfilled, so we have to solve the equations above in several iteration steps. In order to do this we used the Taylorian expansions of Eqs. (25), (26) with respect to $e$. Our calculation is listed in Appendix [A Here we give only the final forms up to the third order in the inner eccentricity, together with such assumption that the other quantities given above are also in the first order of eccentricity. So, in this approximation the modified angular velocity of the apsidal advance, the inner eccentricity, and the argument of periastron in the dynamical frame $(g)$ become

$$
\begin{aligned}
\left(\Pi^{*}\right)^{-1} & =\Pi^{-1}\left(1-\frac{1}{16} A_{2} \mathcal{E}^{2}-\frac{1}{2} A_{1} E \mathcal{E}-A_{1} \epsilon_{0}-\frac{1}{2} A_{2} \epsilon_{0}^{2}\right), \\
e= & e_{0}\left(1+\epsilon_{0}+\frac{1}{4} E \mathcal{E}-\frac{1}{8} A_{1} \mathcal{E}^{2}\right) \\
& -e_{0}\left\{\frac{1}{2}\left[1-\frac{1}{32} \mathcal{E}^{2}+\frac{1}{16} E \mathcal{E}+\frac{1}{4} E^{2}+\left(1-A_{1}\right) \epsilon_{0}\right] \mathcal{E} \cos 2 \mathcal{G}\right. \\
& -\left[\frac{1}{16}\left(1-A_{1}\right) \mathcal{E}+\frac{1}{8} E+\left(\frac{1}{16} \mathcal{E}+\frac{1}{8} E\right) \epsilon_{0}\right] \mathcal{E} \cos 4 \mathcal{G}
\end{aligned}
$$

$$
\begin{aligned}
& \left.+\left[\frac{1}{192} \mathcal{E}^{2}+\frac{1}{32} E \mathcal{E}+\frac{1}{24} E^{2}\right] \mathcal{E} \cos 6 \mathcal{G}\right\}, \\
g= & \mathcal{G}+\frac{1}{2}\left(1+\frac{1}{4} E^{2}\right) E \sin 2 \mathcal{G}+\frac{1}{8} E^{2} \sin 4 \mathcal{G} \\
& +\frac{1}{24} E^{3} \sin 6 \mathcal{G} \\
& -\left[\frac{1}{4} A_{1} \mathcal{E}+\left[\frac{1}{4}\left(A_{1}+A_{2}\right) \mathcal{E}+\frac{1}{2} A_{1} E\right] \epsilon_{0}\right\} \sin 2 \mathcal{G} \\
& +\left[\frac{1}{64}\left(A_{1}+A_{2}\right) \mathcal{E}^{2}-\frac{3}{32} A_{1} E \mathcal{E}\right] \sin 4 \mathcal{G},
\end{aligned}
$$

where

$\mathcal{G}=\Pi^{*}\left(u-u_{0}^{*}\right)$,

while

$$
\begin{aligned}
\epsilon_{0}= & \frac{1}{8}\left(1-A_{1}\right) \mathcal{E}^{2}+\left[\frac{1}{2}+\frac{3}{64} \mathcal{E}^{2}-\frac{1}{32} E \mathcal{E}+\frac{1}{8} E^{2}\right] \mathcal{E} \cos 2 g_{0} \\
& +\left[\frac{1}{16}\left(1-A_{1}\right) \mathcal{E}-\frac{1}{8} E\right] \mathcal{E} \cos 4 g_{0} \\
& +\left[\frac{1}{192} \mathcal{E}^{2}-\frac{1}{32} E \mathcal{E}+\frac{1}{24} E^{2}\right] \mathcal{E} \cos 6 g_{0} \\
A_{1}= & e_{0} \frac{1}{A} \frac{\mathrm{d} A}{\mathrm{~d} e} \\
A_{2}= & e_{0}^{2} \frac{1}{A} \frac{\mathrm{d}^{2} A}{\mathrm{~d} e^{2}}
\end{aligned}
$$

Before continuing with the effects of those quantities which relate to the observational system, we discuss our results for the dynamical apsidal advance rate. First, we consider the instantaneous angular velocity of the apsidal line, i.e $\Pi=\sqrt{A^{2}-B^{2}}$. As we are concentrating on hierarchical triple systems, where the total angular momentum is highly concentrated in the wider orbit, we omit the terms in $A$ and $B$ which are multiplied by $C_{1} / C_{2}$. In this case we can easily have that up to $i_{\mathrm{m}}=63.43$ (i.e. $I^{2}>0.2$ ) $A>A_{\mathrm{r} 3}$. So, when

$A_{\mathrm{r} 2}+A_{\mathrm{n} 1}+A_{\mathrm{n} 2}>A_{\mathrm{t}}-A_{\mathrm{n} 2}$

then the third body affected instantaneous apsidal angular velocity is surely larger, and, consequently, the apsidal motion period is shorter than in the only tidally perturbed case. Note, that Eq. (90) is also the zero order condition for the occurrence of the so-called Kozai resonance in a mass-point three-body model. This gives $i_{\mathrm{m}}<39.23$ for $e=0$. We now concentrate on the average angular velocity, $\Pi^{*}$. There is only one quantity in the $\Pi / \Pi^{*}$ ratio, Eq. (83) which can be negative, namely $\epsilon_{0}$. Consequently, all of the other terms would produce a shorter average period than the instantaneous one. According to Eq. (87), there is a first order term in $\epsilon_{0}, \frac{1}{2} \mathcal{E} \cos 2 g_{0}$, which may give the largest contribution in the whole $\Pi / \Pi^{*}$ ratio. One can see that we could expect a longer average apsidal motion period than the instantaneous one, when $\cos 2 g_{0} \approx-1$. This happens when the argument of periastron is $g_{0} \approx \pm 90^{\circ}$ in the dynamical system in the moment of the calculation of the orbital element (i.e. at the time of observation). Note that as one can see from e.g. Eq (54), for $g_{=} \pm 90^{\circ}$ the eccentricity takes its maximum value, and as is well-known, the larger the eccentricity the faster the tidally-forced apsidal advance speed, this result is not an unexpected one. 
For the sake of completeness, we give our result for the dynamical nodal regression, although up to third order in $e, E$ it is identical with the Taylorian of Eq. 699):

$h=h_{0}^{*}+H_{0} \mathcal{G}-\frac{1}{2} \frac{1}{\cos i_{1}} \frac{A_{\mathrm{n} 2}}{\Pi^{*}} \sin 2 \mathcal{G}$,

where

$H_{0}=-\frac{1}{\Pi^{*}} \frac{1}{\cos i_{1}}\left\{A_{\mathrm{n} 1}+A_{\mathrm{n} 2}\left(1+\frac{1}{2} E\right)+\frac{1}{4} e_{0} \frac{\mathrm{d} A_{\mathrm{n} 2}}{\mathrm{~d} e} \varepsilon\right\}$.

We emphasize again, that this term gives further, negative contribution to the observable apsidal motion period, through the $h \cos i_{1}$ expression, which is independent of the observable inclination of the system.

At this point we refer to the paper of Hegedüs \& Nuspl (1986). In their work they investigate the possible observational effects of nodal motion forced by inclined stellar rotational axes. Although their treatment is entirely correct, they unfortunately denoted the dynamical node (which is $h$ in the present paper) by $\Omega$, which usually means the longitude of the node in the sky (as used in the present work) in the observational astrophysics. From this notation some misinterpretations occured later. So, when Khodykin (1989) reacts to the previously mentioned paper, and states that the orbital plane precession of an eclipsing binary is unable to significantly distort the observed apsidal motion rate (see also Khaliullin et al. 1991), he is right in the sense of the observed node (see the $\mathrm{d} \Omega \cos i$ term in $\mathrm{d} \omega$ ), but Hegedüs \& Nuspl (1986) consider the dynamical node (the contribution of $\mathrm{d} h \cos i_{1}$ in $\mathrm{d} \omega$ ). We note also, that in the inclined rotation case $i_{1}$ is small, which justifies the omission of the $\cos i_{1}$ multiplicator in the work of Hegedüs \& Nuspl (1986).

\subsubsection{Non edge-on orbits: further observational effects from nodal motion $\left(\omega, u_{\mathrm{m}}\right)$}

We know continue with allowing non-exactly edge-on orbits (i.e. $\cos i \neq 0$ ). We then have to take into account the $\mathrm{d} \Omega \cos i$ terms, too. According to Eq. (31), as far as we omit the small amplitude term $\mathrm{d} i_{1}$ this can be written as a function of $\mathrm{d} h \cos i_{1}$. The detailed calculations together with the results up to fifth order in $e$ and $E, \mathcal{E}$ are given in Appendix $\triangle$ (see Eqs. [A.114- A.120]). Here we list the form of the result up to the fourth order in the secular term, and to the third order in the trigonometric ones. Namely,

$\omega=g+u_{\mathrm{m}}$,

where $g$ is given by (85), while

$$
\begin{aligned}
u_{\mathrm{m}}= & \left(u_{\mathrm{m}}\right)_{0}^{*}+U_{0} \mathcal{G}-\frac{1}{2} \frac{A_{\mathrm{n} 2}}{\Pi^{*}}\left(1+C_{0}\right) \sin 2 \mathcal{G}+C_{1} \sin \left(h_{0}^{*}+H_{0} \mathcal{G}\right) \\
& +\frac{1}{2} C_{2} \sin \left(2 h_{0}^{*}+2 H_{0} \mathcal{G}\right)+\frac{1}{3} C_{3} \sin \left(3 h_{0}^{*}+3 H_{0} \mathcal{G}\right),
\end{aligned}
$$

where

$U_{0}=-\frac{1}{\Pi^{*}}\left\{A_{\mathrm{n} 1}+A_{\mathrm{n} 2}\left(1+\frac{1}{2} E\right)+\frac{1}{4} e_{0} \frac{\mathrm{d} A_{\mathrm{n} 2}}{\mathrm{~d} e} \mathcal{E}\right\}\left(1+C_{0}\right)$.

The $C_{0-3}$ terms are coming from the Taylorian expansion of $\left(1-\cos ^{2} i\right)^{-1}$ which arises in the equation for $\mathrm{d} \Omega \cos i$. These are trigonometric functions of $I_{0}$ and $i_{1}$. They are listed only in Appendix A Eqs. A.86- A.113, A.121, A.122). In our calculations we consider $C_{n}$ as it would be $n$-th order in $e$.
Here we would like to stress that in this case a further important difference to those mentioned earlier is that there will no longer be one true period for the apsidal advance in the observational system, now we can speak only about only quasiperiodicity. (This latter statement applies even better for higher orders where the different linear combinations of the angular velocities of $g$ and $h$ appear.) One can see this clearly in Fig. 2, where we plotted the variations of the eccentricity and the observable argument of periastron $(\omega)$ in a hypothetical eccentric triple system for two different initial mutual inclinations $\left(i_{\mathrm{m}}=20^{\circ}, 60^{\circ}\right)$ of the close and the wide orbital planes. (The initial parameters are listed in Tables 1 and 2]) In these figures we connected the $\omega=2 \pi$ values with the corresponding eccentricity values by dashed lines. Here it is clearly visible, that the periods are not equal to the corresponding $e$ and $\omega$. It can be seen also that in the case of $i_{\mathrm{m}}=20^{\circ}$ (left panel) the eccentricity obtained from numerical integration (see next section) significantly departs from the analytical value, while the argument of periastron $(\omega)$ shows a good fit, at least as far as one omits the small cumulative error in the period. On the contrary in the case of $i_{\mathrm{m}}=60^{\circ}$ (right panel) eccentricity curves produce very satisfactory fits, while the analytical $\omega$ departs from the numerical curve suddenly after approx. 1000 years. In the first case, the explanation can be found in the small mutual inclination, in which case, as was mentioned earlier, the higher order terms in the perturbative forces are also important, while in the second case the departure arises from the fact, that due to the large amplitude orbital precession the $\cos i^{-1}$ term becomes so large that our approximation will no longer be satisfactory.

\subsubsection{Direct perturbations in the orbital motion}

We also calculate the direct terms for higher accuracy. This is slightly problematic, as due to the very strong $e$-dependence, the derivatives of $A_{\mathrm{d}}$ could increase to very large values already for medium eccentricities. Fortunately, if the time differences of the two types of minima are used instead of the usual $\mathrm{O}-\mathrm{C}$ function, these direct terms will fall out, as at the same time they have the same value for primary and secondary minima. Nevertheless, we use them in the following, considering the quantities derived from the derivatives of $A_{\mathrm{d}}$ as first order, and those from $B_{\mathrm{d}}$ as second order (in our present sample configuration of AS Cam this can be done approx. up to $e^{\prime}=0.3$ for perpendicular orbits). The results up to fifth order can be found in Eqs. A.70- A.75, while up to third order are listed below:

$$
\begin{aligned}
\delta= & \delta_{0}^{*}+D_{0} \mathcal{G} \\
& +\left\{-\frac{1}{4}\left[V_{1}\left(1+\epsilon_{0}\right)+V_{2} \epsilon_{0}\right] \mathcal{E}+\frac{1}{2} W\left(1+\epsilon_{0}\right)\right\} \sin 2 \mathcal{G} \\
& +\left[\frac{1}{64}\left(V_{1}+V_{2}\right) \mathcal{E}^{2}-\frac{1}{32} V_{1} E \mathcal{E}-\frac{1}{16} W_{1} \mathcal{E}+\frac{1}{8} W E\right] \sin 4 \mathcal{G},
\end{aligned}
$$

where

$$
\begin{aligned}
D_{0}= & \frac{1}{\Pi^{*}}\left[A_{\mathrm{d}}+e_{0} \frac{\mathrm{d} A_{\mathrm{d}}}{\mathrm{d} e}\left(\epsilon_{0}+\frac{1}{4} E \mathcal{E}\right)+e_{0}^{2} \frac{\mathrm{d}^{2} A_{\mathrm{d}}}{\mathrm{d} e^{2}}\left(\frac{1}{16} \mathcal{E}^{2}+\frac{1}{2} V_{2} \epsilon_{0}^{2}\right)\right. \\
& \left.-\frac{1}{2} B_{\mathrm{d}} E-\frac{1}{4} e_{0} \frac{\mathrm{d} B_{\mathrm{d}}}{\mathrm{d} e}\left(\epsilon_{0}+\mathcal{E}\right)-\frac{1}{4} e_{0}^{2} \frac{\mathrm{d}^{2} B_{\mathrm{d}}}{\mathrm{d} e^{2}} \epsilon_{0}\right],
\end{aligned}
$$

while

$$
V=\frac{1}{\Pi^{*}} A_{\mathrm{d}},
$$



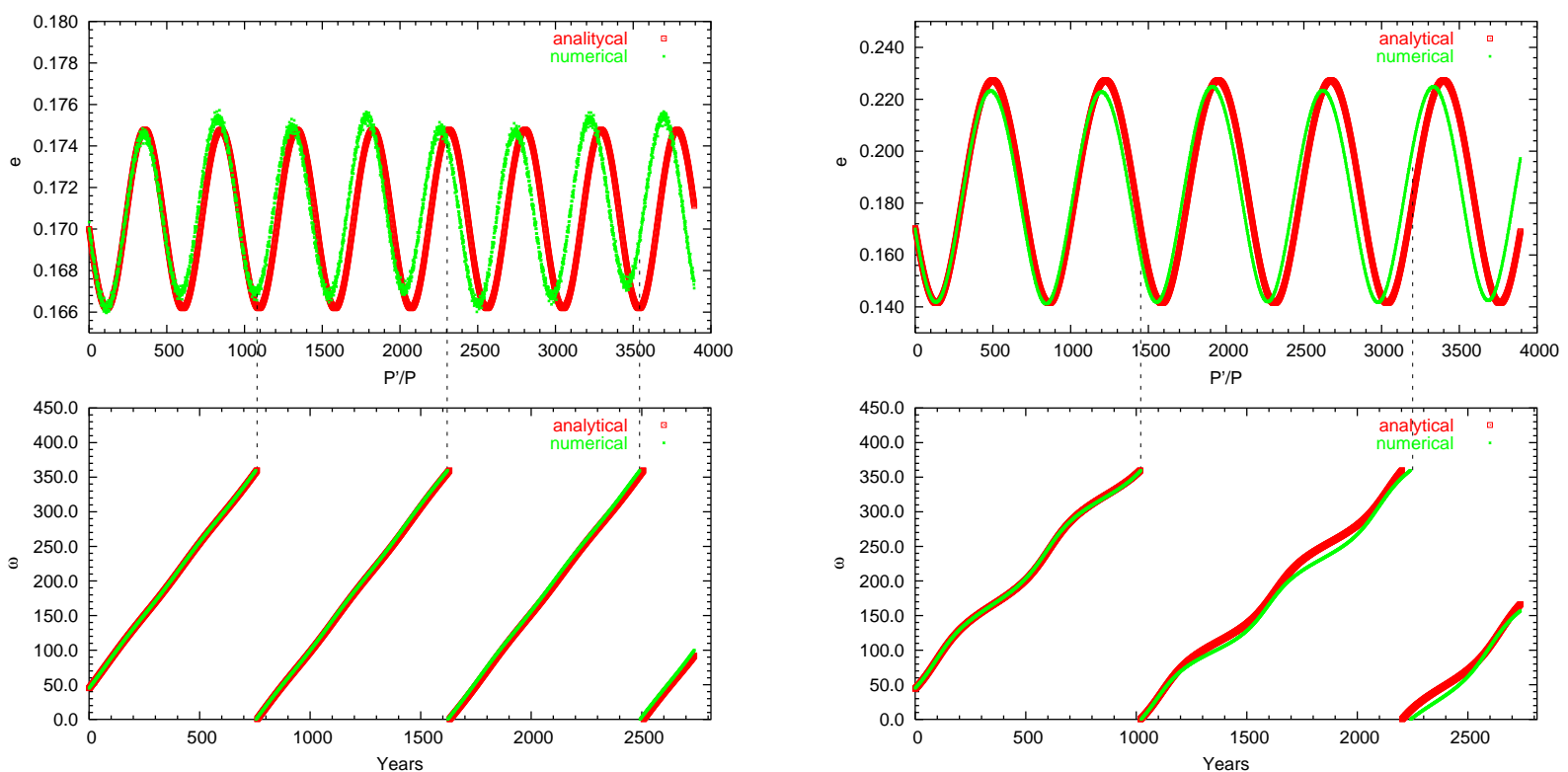

Fig. 2. The variation of the eccentricity and the observable argument of periastron in the case of the AS Camelopardalis due to the perturbations of a third body. The initial mutual inclinations are $i_{\mathrm{m}}=20^{\circ}$ (left panel), and $i_{\mathrm{m}}=60^{\circ}$ (right panel). The dashed lines connect the $\omega=2 \pi$ values with the corresponding eccentricity, illustrating that the period of the $e$-cycles and the observable apsidal motion is different. (See text for an explanation of the discrepancy between the numerical and analytical results.)

$$
\begin{aligned}
V_{1} & =e_{0} \frac{1}{\Pi^{*}} \frac{\mathrm{d} A_{\mathrm{d}}}{\mathrm{d} e}, \\
V_{2} & =e_{0}^{2} \frac{1}{\Pi^{*}} \frac{\mathrm{d}^{2} A_{\mathrm{d}}}{\mathrm{d} e^{2}}, \\
W & =\frac{1}{\Pi^{*}} B_{\mathrm{d}}, \\
W_{1} & =e_{0} \frac{1}{\Pi^{*}} \frac{\mathrm{d} B_{\mathrm{d}}}{\mathrm{d} e}, \\
W_{2} & =e_{0}^{2} \frac{1}{\Pi^{*}} \frac{\mathrm{d}^{2} B_{\mathrm{d}}}{\mathrm{d} e^{2}} .
\end{aligned}
$$

\subsubsection{Generalized form of the $\mathrm{O}-\mathrm{C}$}

Finally, now we report the generalized form of the $\mathrm{O}-\mathrm{C}$ curve, which is as follows:

$$
\begin{aligned}
\frac{2 \pi}{P} O-C \approx & V_{100} \cos \left[\omega_{0}^{*}+\left(1+U_{0}\right) \mathcal{G}\right] \\
& +V_{200} \sin \left[2 \omega_{0}^{*}+\left(2+2 U_{0}\right) \mathcal{G}\right] \\
& +V_{300} \cos \left[3 \omega_{0}^{*}+\left(3+3 U_{0}\right) \mathcal{G}\right] \\
& +V_{1-20} \cos \left[\omega_{0}^{*}-\left(1-U_{0}\right) \mathcal{G}\right] \\
& +V_{120} \cos \left[\omega_{0}^{*}+\left(3+U_{0}\right) \mathcal{G}\right] \\
& +V_{1-40} \cos \left[\omega_{0}^{*}-\left(3-U_{0}\right) \mathcal{G}\right] \\
& +V_{140} \cos \left[\omega_{0}^{*}+\left(5+U_{0}\right) \mathcal{G}\right] \\
& +V_{2-20} \sin \left[2 \omega_{0}^{*}+2 U_{0} \mathcal{G}\right] \\
& +V_{220} \sin \left[2 \omega_{0}^{*}+\left(4+2 U_{0}\right) \mathcal{G}\right] \\
& +V_{10-1} \cos \left[\omega_{0}^{*}-h_{0}^{*}+\left(1+U_{0}-H_{0}\right) \mathcal{G}\right] \\
& +V_{101} \cos \left[\omega_{0}^{*}+h_{0}^{*}+\left(1+U_{0}+H_{0}\right) \mathcal{G}\right] \\
& +V_{10-2} \cos \left[\omega_{0}^{*}-2 h_{0}^{*}+\left(1+U_{0}-2 H_{0}\right) \mathcal{G}\right] \\
& +V_{102} \cos \left[\omega_{0}^{*}+2 h_{0}^{*}+\left(1+U_{0}+2 H_{0}\right) \mathcal{G}\right] \\
& +V_{1-2-1} \cos \left[\omega_{0}^{*}-h_{0}^{*}-\left(1-U_{0}+H_{0}\right) \mathcal{G}\right] \\
& +V_{1-21} \cos \left[\omega_{0}^{*}+h_{0}^{*}-\left(1-U_{0}-H_{0}\right) \mathcal{G}\right]
\end{aligned}
$$

$$
\begin{aligned}
& +V_{12-1} \cos \left[\omega_{0}^{*}-h_{0}^{*}+\left(3+U_{0}-H_{0}\right) \mathcal{G}\right] \\
& +V_{121} \cos \left[\omega_{0}^{*}+h_{0}^{*}+\left(3+U_{0}+H_{0}\right) \mathcal{G}\right] \\
& +V_{20-1} \sin \left[2 \omega_{0}^{*}-h_{0}^{*}+\left(2+2 U_{0}-H_{0}\right) \mathcal{G}\right] \\
& +V_{201} \sin \left[2 \omega_{0}^{*}+h_{0}^{*}+\left(2+2 U_{0}+H_{0}\right) \mathcal{G}\right] \\
& +V_{020} \sin 2 \mathcal{G} \\
& +V_{040} \sin 4 \mathcal{G} \\
& +V_{001} \sin \left[h_{0}^{*}+H_{0} \mathcal{G}\right] \\
& +V_{002} \sin \left[2 h_{0}^{*}+2 H_{0} \mathcal{G}\right] \\
& +V_{003} \sin \left[3 h_{0}^{*}+3 H_{0} \mathcal{G}\right]+O\left(e^{4}, E^{4}\right),
\end{aligned}
$$

where

$$
\begin{aligned}
V_{100} & =-2 j e_{0}\left(1-\frac{1}{4} C_{1}^{2}\right)\left(1+\epsilon_{0}\right), \\
V_{1-20} & =j e_{0}\left[\frac{1}{2}(\mathcal{E}+E)\left(1+\epsilon_{0}\right)-\frac{1}{4} A_{1} \mathcal{E}\right], \\
V_{120} & =j e_{0}\left[\frac{1}{2}(\mathcal{E}-E)\left(1+\epsilon_{0}\right)+\frac{1}{4} A_{1} \mathcal{E}\right], \\
V_{1-40} & =\frac{1}{16} j e_{0}\left(-\mathcal{E}^{2}+E^{2}\right), \\
V_{140} & =j e_{0}\left(-\frac{1}{16} \mathcal{E}^{2}+\frac{1}{4} E \mathcal{E}-\frac{3}{16} \mathcal{E}^{2}\right), \\
V_{200} & =\frac{3}{4} e_{0}^{2}\left(1+2 \epsilon_{0}\right), \\
V_{2-20} & =-\frac{3}{8} e^{2}(\mathcal{E}+E), \\
V_{220} & =\frac{3}{8} e^{2}(-\mathcal{E}+E),
\end{aligned}
$$




$$
\begin{aligned}
V_{300} & =\frac{1}{3} j e_{0}^{3} \\
V_{10 \pm 1} & =\mp j e_{0} C_{1}\left(1+\epsilon_{0}\right) \\
V_{10-2} & =-\frac{1}{4} j e_{0}\left(C_{1}^{2}-2 C_{2}\right) \\
V_{102} & =\frac{1}{4} j e_{0}\left(C_{1}^{2}+2 C_{2}\right) \\
V_{1-2 \pm 1} & =\mp \frac{1}{4} j e_{0} C_{1}(\mathcal{E}+E) \\
V_{12 \pm 1} & = \pm \frac{1}{4} j e_{0} C_{1}(\mathcal{E}-E) \\
V_{20 \pm 1} & = \pm \frac{3}{4} e_{0}^{2} C_{1}, \\
V_{020} & =-\frac{1}{4}\left[V_{1}\left(1+\epsilon_{0}\right)+V_{2} \epsilon_{0}\right] \mathcal{E}+\frac{1}{2} W\left(1+\epsilon_{0}\right)+\frac{1}{2} M C_{0}, \\
V_{040} & =\frac{1}{64}\left(A_{1}+A_{2}\right) \mathcal{E}^{2}-\frac{3}{32} A_{1} E \mathcal{E} \\
V_{00 n} & =-\frac{1}{n} C_{n} .
\end{aligned}
$$

Furthermore,

$$
M=\frac{1}{\Pi^{*}} A_{\mathrm{n} 2} .
$$

A more detailed result up to fifth order containing 102 trigonometric terms is also listed in Appendix A Eqs. A.123 - A.225. Here we note, that the indices refer to the multiplicators of $\omega, g$, and $h$ in the trigonometric terms, respectively. We separated the different terms by blank lines. All four groups besides the perturbations in $e$ and $g$, give the additional contribution of the precession of the orbital plane with respect to the observer 2 The first, and second groups give the nodal contribution to the observable argument of periastron $(\omega)$, while the third one comes both directly from the $\Omega \cos i$, and the direct orbiatl motion terms in $\dot{u}$. Finally, the fourth one also contains the contribution of $\Omega \cos i$. These latter two terms, naturally have $e$-independent parts, consequently they do not disappear even in the case of a circular inner orbit. Nevertheless, as was mentioned above, due to the $\cos i$ multiplicator, their significance is very limited in the case of the relatively wider eccentric eclipsing binaries. Similarly, due to this latter condition, the contribution of the second group is also very minimal, as these terms arise exactly from the same reason as the terms of the fourth groups, manifesting the same precession indirectly, through the variation of $\omega$. On the contrary, the first group contains such terms of the nodal motion which remain effective even in the edge-on $\cos i=0$ case, too. These are coming from the $h \cos i_{1}$ term as was discussed in Sects. 2.2.2 and 2.3.1.

To illustrate the net effect to the $\mathrm{O}-\mathrm{C}$ we plotted in Fig. 3 the $\mathrm{O}-\mathrm{C}$ diagram of AS Camelopardalis for the same two configurations for which the variations of $e$ and $\omega$ were shown in Fig. 22 The upper panels represent the numerical results derived directly from the numerical integrations as well as analytical results calculated according to the higher order formula listed in Appendix A Eq. A.123). The lower panels represent the numerical curve and the "unperturbed" (i.e. only two-body tidal distortion is present, as usual) theoretical curve where both the

\footnotetext{
${ }^{2}$ We do not use intentionally the usual phrase 'nodal regression', as we concentrate on the observational frame of reference, where we can observe even nodal progression (see Sect. 33.
}

constant eccentricity and apsidal motion period were deduced from the "measured" $e_{0}=e\left(t_{0}\right)$ value. We note, that the significant departure of the numerical and the analytical curves which occurs in the $i_{\mathrm{m}}=60^{\circ}$ case after approximately 700 years (upper right panel) should arise from the fact that due to the variation of the observable inclination $(i)$ for this time our fundamental principle, i.e. Eq. (1) ceases to be correct. Nevertheless, at this value of $i$, our system is already no longer an eclipsing one, consequently, this situation is out of our scope.

\subsection{Discussion on observational effects}

When only a small fraction of the O-C curve is observed, several serious problems occur in the sense of the determination of the apsidal motion period with respectable accuracy. To demonstrate this we consider only the $V_{100}$ and $V_{1-20}$ terms (we omit $V_{120}$ because we concentrate mainly on such configurations where the mutual inclination of the inner and the outer orbit is large, and consequently, we suppose that $\mathcal{E} \approx E$ ). Furthermore, when the period of the nodal motion is significantly longer than the tidally induced apsidal motion (as in the case of AS Cam, but not necessarily in e.g. DI Her), then $u_{\mathrm{m}}$, as well as $h$ can be considered as constant. Then instead of the usual O-C we apply the difference function of the primary and secondary minima, as

$$
\begin{aligned}
\Delta \approx & \frac{P}{\pi} e_{0}\left\{-2 \cos \left[\omega_{0}^{*}+\mathcal{G}_{0}+\Pi^{*}\left(u-u_{0}\right)\right]\right. \\
& \left.+E \cos \left[\omega_{0}^{*}-\mathcal{G}_{0}-\Pi^{*}\left(u-u_{0}\right)\right]\right\} .
\end{aligned}
$$

As far as the nodal motion is neglected it can easily be seen that

$\omega_{0}^{*}=u_{\mathrm{m}}$,

furthermore, according to its definition

$\mathcal{G}_{0}=g_{0}-\frac{1}{2} E \sin 2 g_{0}+\ldots$,

so,

$\omega_{0}^{*}+\mathcal{G}_{0} \approx \omega_{0}-\frac{1}{2} E \sin 2 g_{0}$,

$\omega_{0}^{*}-\mathcal{G}_{0} \approx \omega_{0}-2 g_{0}+\frac{1}{2} E \sin 2 g_{0}$.

We concentrate continuously on perpendicular orbital planes configuration. In this case, taking into account that the binary is an eclipsing one, i.e. its observable inclination is close to $90^{\circ}$, the plane of the outer orbit should lie either close to the plane of the sky, or perpendicular to both the planes of the inner orbit and the sky. In the previous case (which is the more interesting one, because it makes it possible not to observe light-time effect) it is easy to see that $\omega \approx g+[0,1] \times \pi$, while in the latter one $\omega \approx g \pm \pi / 2$ (we also used the fact that in these hierarchical systems the outer plane is close to the invariable plane). In the two different situations we get that

$$
\begin{aligned}
\Delta_{0, \pi} & \approx-2 \frac{P}{\pi} e_{0}\left(1+\frac{1}{2} \mathcal{E} \cos 2 \omega_{0}-\frac{1}{2} \mathcal{E}\right) \cos \left[\omega_{0}+\Pi^{*}\left(u-u_{0}\right)\right] \\
\Delta_{ \pm \pi / 2} & =-2 \frac{P}{\pi} e_{0}\left(1-\frac{1}{2} \mathcal{E} \cos 2 \omega_{0}+\frac{1}{2} \mathcal{E}\right) \cos \left[\omega_{0}+\Pi^{*}\left(u-u_{0}\right)\right]
\end{aligned}
$$

The subscript of $\Delta$ refers to the approximate value of $u_{\mathrm{m}}$ and we omitted the $\sin 2 g_{0}$ terms inside the arguments. Eq. (129) 

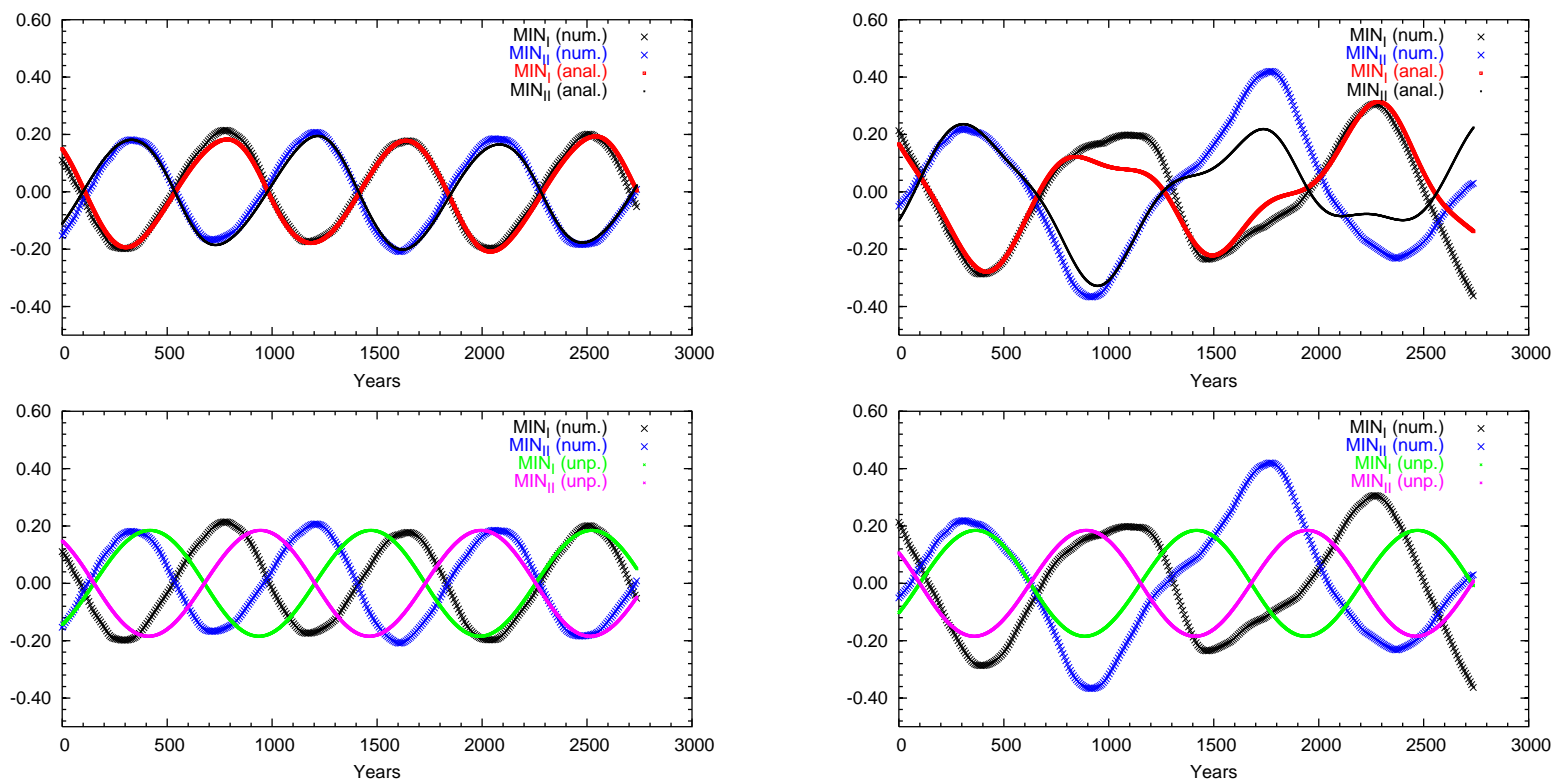

Fig. 3. The numerically (AS2 and AS3 runs - see next section) and analytically generated O-C curve in the case of the AS Camelopardalis with the perturbations of a third body. The initial mutual inclinations are $i_{\mathrm{m}}=20^{\circ}$ (left panel), and $i_{\mathrm{m}}=60^{\circ}$ (right panel). Upper panels show the numerically generated O-Cs, as well as the analytical ones calculated according to the formulae of the present paper. Lower panels demonstrate the difference between the numerical (i.e. "observed") curves, and the only tidally forced apsidal motion produced $\mathrm{O}-\mathrm{C}$.
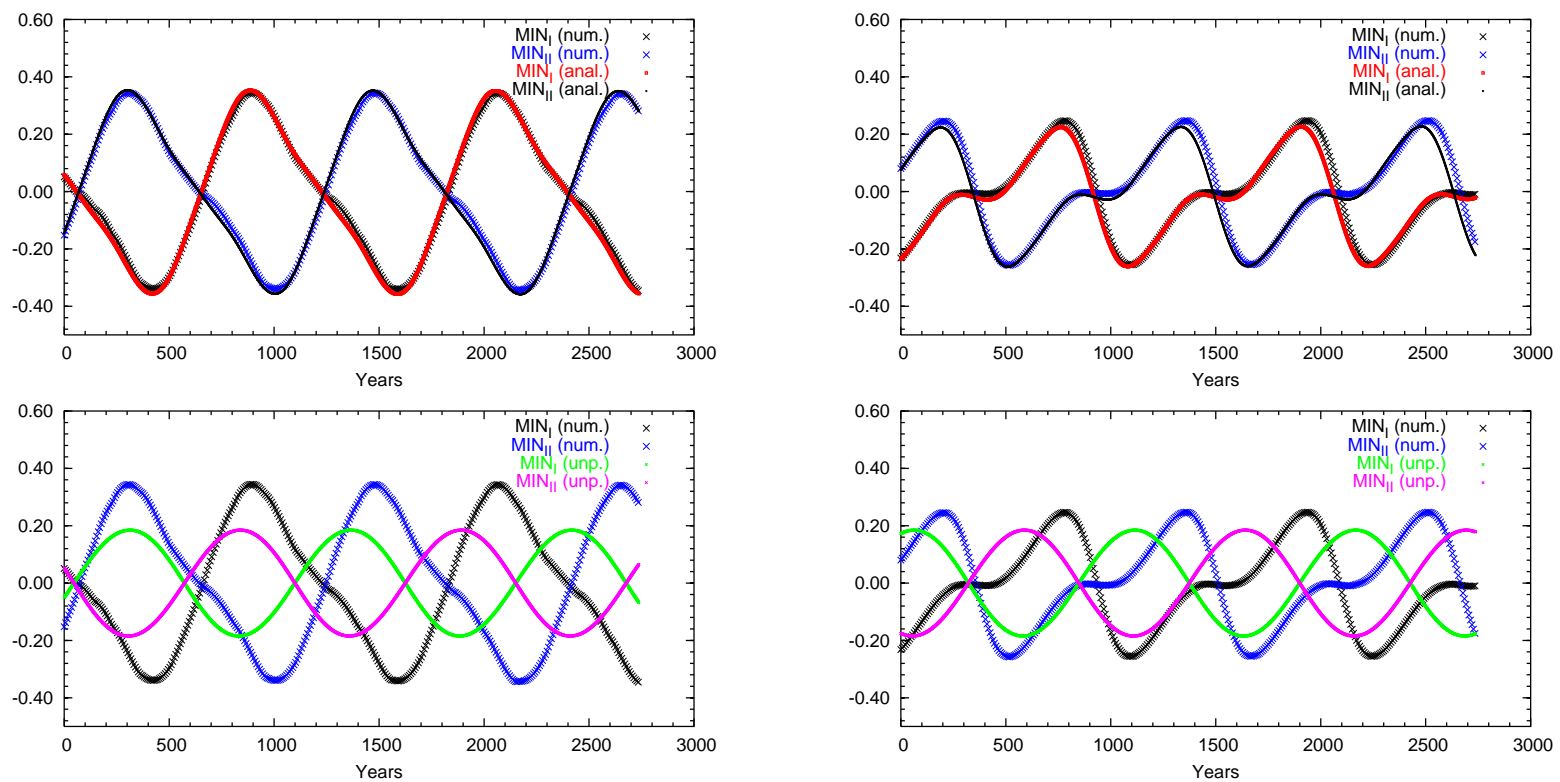

Fig. 4. As in Fig. 3, but the initial mutual inclinations are $i_{\mathrm{m}} \approx 90^{\circ}$ for both panels (AS4 and AS4b runs - see next section). The only difference is that in the left panel the orbital plane of the tertiary is almost perpendicular to the plane of the sky, while in the right panel this nearly coincides with it. Note that at this second configuration the amplitude of the $\mathrm{O}-\mathrm{C}$ is definitely smaller than in the first case.

shows a very important result. In the situation when a third body revolves around the eclipsing binary in a nearly perpendicular plane which lies close to the plane of the sky, the amplitude of the $\Delta$ curve - which is used several times for the calculation of the period of the apsidal motion - could be lower than it is expected (by the multiplicator $0 \geq \delta \geq 1-\mathcal{E}$ ), consequently, the numerical fitting will result in a smaller angular velocity, i.e. longer period. This is demonstrated in Figs. 4 and 5, where two numerically integrated $\mathrm{O}-\mathrm{C}$ curves are shown (together with the corresponding analytically calculated ones). There is no dif- ference in the physical configuration of the entire triple system, only its orientation rotated with $90^{\circ}$ along the inner orbital plane, i.e. the outer orbital plane from an almost perpendicular position became nearly coincident with the plane of the sky. Moreover, besides the smaller amplitude, Fig. 5 reveals a more important result. As one can see, when the orbital plane of the tertiary is close to the plane of the sky, large almost horizontal regions can be found in the difference curve. (This means that in these intervals the primary and the secondary minima vary almost in the same manner.) The mathematical cause can be understood from 


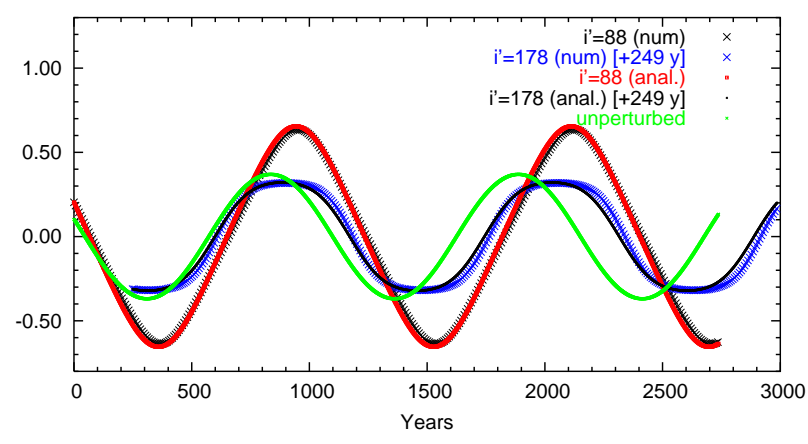

Fig. 5. The difference of the $\mathrm{O}-\mathrm{C}$ of the primary and the secondary minima for the $\mathrm{O}-\mathrm{Cs}$ plotted in Fig. 4 We shifted the $i^{\prime}=178^{\circ}$ curve with approx. 249 years in order to coincide with the "unperturbed" curve of the two cases.

the third order approximation. In this case the $V_{100}, V_{1 \pm 20}$ and the $V_{300}$ terms should be counted. Limiting ourselves only for the third-body-in-the-sky case, then

$$
\begin{aligned}
\Delta_{0, \pi}= & -2 \frac{P}{\pi} e_{0}\left[\left(1-\frac{1}{2} \mathcal{E}+\frac{1}{2} \mathcal{E} \cos 2 \omega_{0}+\frac{1}{8} \mathcal{E}^{2}\right.\right. \\
& \left.-\frac{1}{4} \mathcal{E}^{2} \cos 2 \omega_{0}+\frac{1}{16} \mathcal{E}^{2} \cos 4 \omega_{0}+\frac{1}{8} A_{1} \mathcal{E}\right] \cos \mathcal{G} \\
& \left.-\left(\frac{1}{8} A_{1} \mathcal{E}+\frac{1}{6} e_{0}^{2}\right) \cos 3 \mathcal{G}\right] .
\end{aligned}
$$

The flat extrema occur when $\mathcal{G}=0+k \pi$. Around these intervals the two cosine terms have opposite signs. This also happens in the "unperturbed" case of course. However, a significant difference is that, whilst we considered both $A_{1}$ and $\mathcal{E}$ as formally having the same order than $e$, both could be somewhat larger than $e$, and in some cases they can reach almost the order of $e^{1 / 2}$. Consequently, the superposition of the opposite sign $\cos 3 \mathcal{G}$ curve onto the $\cos \mathcal{G}$ may cause further flattening almost in the order of $e$. These areas might cover even the $40-50 \%$ of the total curve. It is trivial, that if this system were observed within this time interval, the apsidal motion period would be found significantly longer than the theoretically expected value. On the other side, there is no region, where the slope of the difference curve is significantly larger than the "unperturbed" reference curve. The consequences of the fact above will be discussed in Sect. 4 .

We now study numerically the eclipsing system AS Camelopardalis, where only about a $10 \%$ or less of the total period is covered by observations.

\section{Numerical studies}

We carried out several sets of integrations with the numeric integrator described in Borkovits et al. (2004). These runs partly serve as numerical support for the analytical calculations described in the previous section, and partly serve as study on the dynamical evolution of eccentric hierarchical triple systems with and without dissipation. The initial parameters of the binary were taken from Khodykin \& Vedeneyev (1997), with the exception of the inner structure constants which were set according to the tables of Claret \& Gimènez (1991). The physical parameters of the three stars as well as those initial orbital elements which are the same in the different runs are listed in Table1, while those orbital elements which differ in the individual runs are listed in Table 2. In this latter table we give the angular orbital elements
Table 1. Fixed initial parameters of the binary as well as of the tertiary for every run. The $k_{j}^{(i)}$ constants are taken from the tables of Claret \& Gimènez (1991). The other parameters for the binary (with the exception of $\Omega$ which has an arbitrary value) are from Khodykin \& Vedeneyev (1997). The parameters of the third companion (primed quantities) are partly from our earlier light-time solution (see Borkovits 2003).

\begin{tabular}{ll|ll}
\hline \hline$a$ & $17.195 \mathrm{R}_{\odot}$ & $a^{\prime}$ & $736.98 \mathrm{R}_{\odot}$ \\
$e$ & 0.17 & $e^{\prime}$ & 0.41 \\
$\tau^{a}$ & $50000 \mathrm{HJD}$ & $\tau^{\prime}$ & $52085 \mathrm{HJD}$ \\
& 50000.82 & & \\
& 50001.7 & & \\
$i$ & $88^{\circ} .78$ & & \\
$\Omega$ & $130^{\circ}$ & & \\
\hline$m_{1}$ & $3.3 \mathrm{M}_{\odot}$ & $m_{2}$ & $2.5 \mathrm{M}_{\odot}$ \\
$m_{3}$ & $1.1 \mathrm{M}_{\odot}$ & & \\
$R_{1}$ & $2.60 \mathrm{R}_{\odot}$ & $R_{2}$ & $1.96 \mathrm{R}_{\odot}$ \\
$k_{2}^{(1)}$ & 0.0049 & $k_{2}^{(2)}$ & 0.0038 \\
$k_{3}^{(1)}$ & 0.0011 & $k_{3}^{(2)}$ & 0.0008 \\
$\lambda_{1}$ & 0.0 & $\lambda_{2}$ & 0.0 \\
& 0.0001 & & 0.0001 \\
\hline
\end{tabular}

$a$ : The three values refer to departure from periastron, instantaneousangular-velocity-equal-to-average, and apastron positions, respectively.

both in the observational $\left(\omega, \omega^{\prime}, \Omega^{\prime}, i^{\prime}\right)$, and the dynamical $(g$, $\left.h, i_{1}, h^{\prime}, i_{2}\right)$ coordinate systems. Note, our input parameters for the integrator are only the observational angular elements, and the others are only derived quantities. All columns of this latter table refer to four individual runs. One of them is the dissipationless case, starting the binary from its periastron, and three of them with dissipation $\left(\lambda_{1}=\lambda_{2}=10^{-4}\right)$, with initial position of the binary in its periastron, apastron, and at true anomaly $v \approx 104^{\circ}$, where the instantaneous orbital angular velocity in the present configurations is nearly equal to the averaged one (as we used the option of our code which makes the angular velocity vectors of the stellar rotation for both stars equally long and parallel to the initial instantaneous orbital angular velocity vector in an iterative way, it means that the initial rotation of the stars were synchronized in these three different ways.) We emphasize again, that as we were not interested in it exclusively dynamical evolution, but for observational consequences too, we carried out some runs where the dynamics of the triple were the same, but its orientation with respect to the Earth were different (e.g. AS3, AS4 and AS3b, AS4b runs).

\subsection{Non-dissipative runs}

The variation of the orbital elements during the integrations can be seen in Figs. 66 9 These figures represent the dissipationless, departed from periastron runs. The left panels cover a time interval of 100 years, which is naturally briefer than a moment from dynamical point of view, but this is the time interval (in the best scenarios) which can usually be reached for the present studies. The numerically generated $\mathrm{O}-\mathrm{C}$ curve, as well as the variations of the orbital elements $e$ and $\omega$ of the AS2-AS4b runs were also used in the previous section as an illustration for theoretical thinkings (see Figs. 2-5). Additional integrations were performed for the close binary without the tertiary component both in the quasi-synchronized rotator, and the precessing secondary component case (this latter is not presented here). 

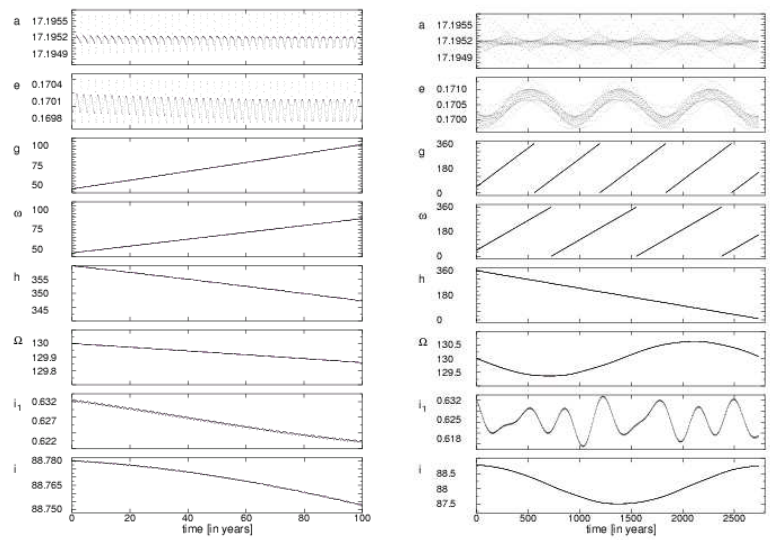

Fig. 6. The variation of both the dynamical and the observational orbital elements of the binary during 100 and approx. 2800 years ( 1 million days). Semi-major axis is given in $R_{\odot}$, while the angular elements in degrees. The mutual inclination is $i_{\mathrm{m}} \approx 0.8$. See Col. AS1 of Table 2 for the initial values.
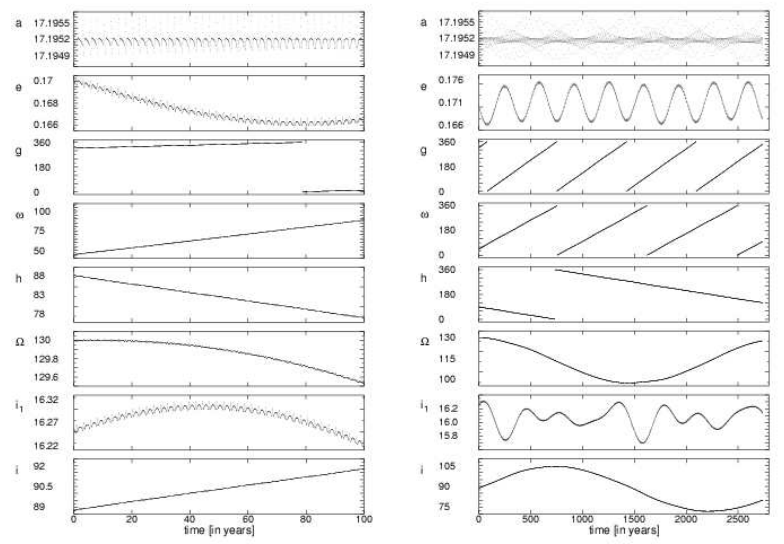

Fig. 7. The variation of both the dynamical and the observational orbital elements of the binary during 100 and approx. 2800 years ( 1 million days). Semi-major axis is given in $R_{\odot}$, while the angular elements in degrees. The mutual inclination is $i_{\mathrm{m}} \approx 20^{\circ}$. See Col. AS2 of Table 2 for the initial values.
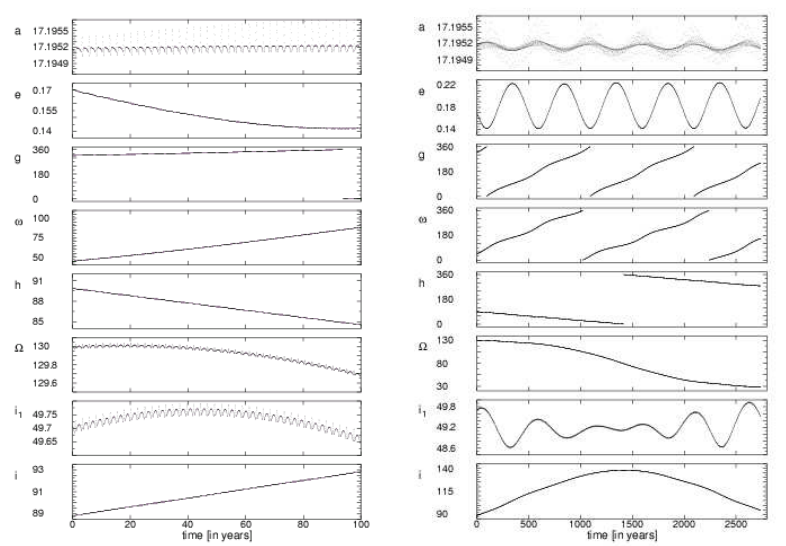

Fig. 8. The variation of both the dynamical and the observational orbital elements of the binary during 100 and approx. 2800 years ( 1 million days). Semi-major axis is given in $R_{\odot}$, while the angular elements in degrees. The mutual inclination is $i_{\mathrm{m}} \approx 60^{\circ}$. See Col. AS3 of Table 2 for the initial values.
Table 2. Initial orbital elements, and some derived quantities, which were different at individual integration runs.

\begin{tabular}{lllllll}
\hline \hline & AS1 & AS2 & AS3 & AS3b & AS4 & AS4b \\
\hline$g$ & $45^{\circ} .0$ & $317^{\circ} .5$ & $316^{\circ} .6$ & $57^{\circ} .1$ & $145^{\circ} .1$ & $327^{\circ} .1$ \\
$\omega$ & $45^{\circ}$ & $45^{\circ}$ & $45^{\circ}$ & $57^{\circ} .1$ & $57^{\circ} .1$ & $147^{\circ} .1$ \\
$h$ & 0.00 & $87^{\circ} .94$ & $89^{\circ} .89$ & $0^{\circ} .00$ & $269^{\circ} .27$ & $180^{\circ} .00$ \\
$i_{1}$ & 0.63 & $16^{\circ} .25$ & $49^{\circ} .71$ & $49^{\circ} .74$ & $76^{\circ} .80$ & $76^{\circ} .76$ \\
\hline$g^{\prime}$ & $81^{\circ} .00$ & $352^{\circ} .93$ & $350^{\circ} .75$ & $351^{\circ} .00$ & $172^{\circ} .22$ & $351^{\circ} .00$ \\
$\omega^{\prime}$ & $261^{\circ}$ & $261^{\circ}$ & $261^{\circ}$ & $171^{\circ}$ & $261^{\circ}$ & $351^{\circ}$ \\
$h^{\prime}$ & $179^{\circ} .87$ & $267^{\circ} .95$ & $269^{\circ} .89$ & $180^{\circ} .00$ & $89^{\circ} .27$ & $0^{\circ} .00$ \\
$\Omega^{\prime}$ & $130^{\circ}$ & $110^{\circ}$ & $70^{\circ}$ & $130^{\circ}$ & $220^{\circ}$ & $130^{\circ}$ \\
$i_{2}$ & 0.15 & $3^{\circ} .75$ & $10^{\circ} .27$ & $10^{\circ} .26$ & $13^{\circ} .16$ & $13^{\circ} .16$ \\
$i^{\prime}$ & $88^{\circ} .0$ & $88^{\circ} .0$ & $88^{\circ} .0$ & $28^{\circ} .78$ & $88^{\circ} .0$ & $178^{\circ} .7$ \\
\hline$i_{\mathrm{m}}$ & 0.78 & $20^{\circ} .01$ & $59^{\circ} .98$ & $60^{\circ} .00$ & $89^{\circ} .96$ & $89^{\circ} .92$ \\
\hline
\end{tabular}
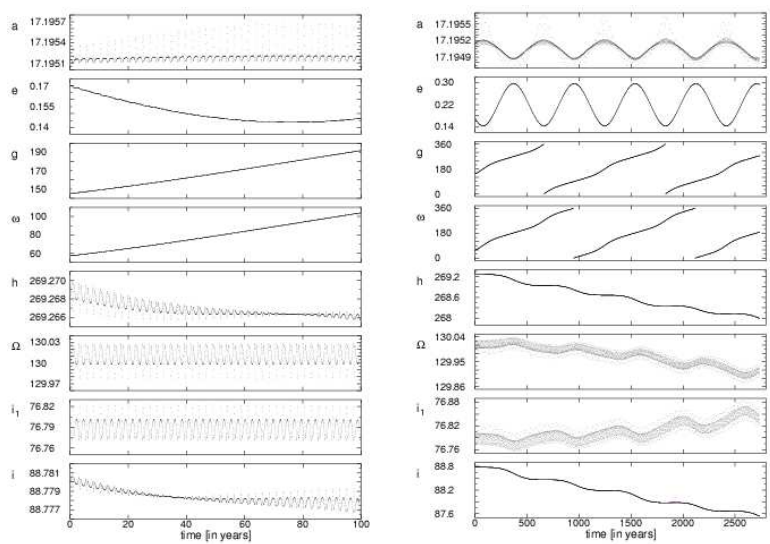

Fig. 9. The variation of both the dynamical and the observational orbital elements of the binary during 100 and approx. 2800 years (1 million days). Semi-major axis is given in $R_{\odot}$, while the angular elements in degrees. The mutual inclination is $i_{\mathrm{m}} \approx 90^{\circ}$. See Col. AS4 of Table 2 for the initial values.

In the quasi-synchronized binary case $U=381800 \mathrm{~d} \approx$ $1046 \mathrm{y}$ was found for the period of the apsidal motion without the relativistic contribution. This yields $\dot{\omega}_{\mathrm{cl}}=\dot{g}_{\mathrm{cl}}=34^{\circ} .4 / 100 \mathrm{y}$ for the classical part of the apsidal advance rate, which is in good agreement with the theoretical value of Maloney et al. (1989) $\dot{g}_{\mathrm{cl}}=35.8 \pm 5.8 / 100 \mathrm{y}$.

In Fig. 10 we plotted the variation of the apsidal line both in the observational $(\omega)$, and in the dynamical $(g)$ frame of references for five different configurations of the system. Comparing the four third-body perturbed runs with the quasi-synchronized binary configuration, one can see that there are no cases where the apsidal motion period would be significantly longer than in the reference case. However, on the contrary, for the low mutual inclination cases (AS1, AS2), the apsidal motion period is found to be remarkably smaller. This is in good correspondance with our discussion on $\left(\Pi^{*}\right)^{-1}$ in Sect. 2.3.1. Consequently, we can conclude again, that the observational effects due to the specific spatial configuration of the orbital plane of the perturbing third star should play a more important role in the explanation of the anomalously slow apsidal motion than the dynamical perturbations themselves. 

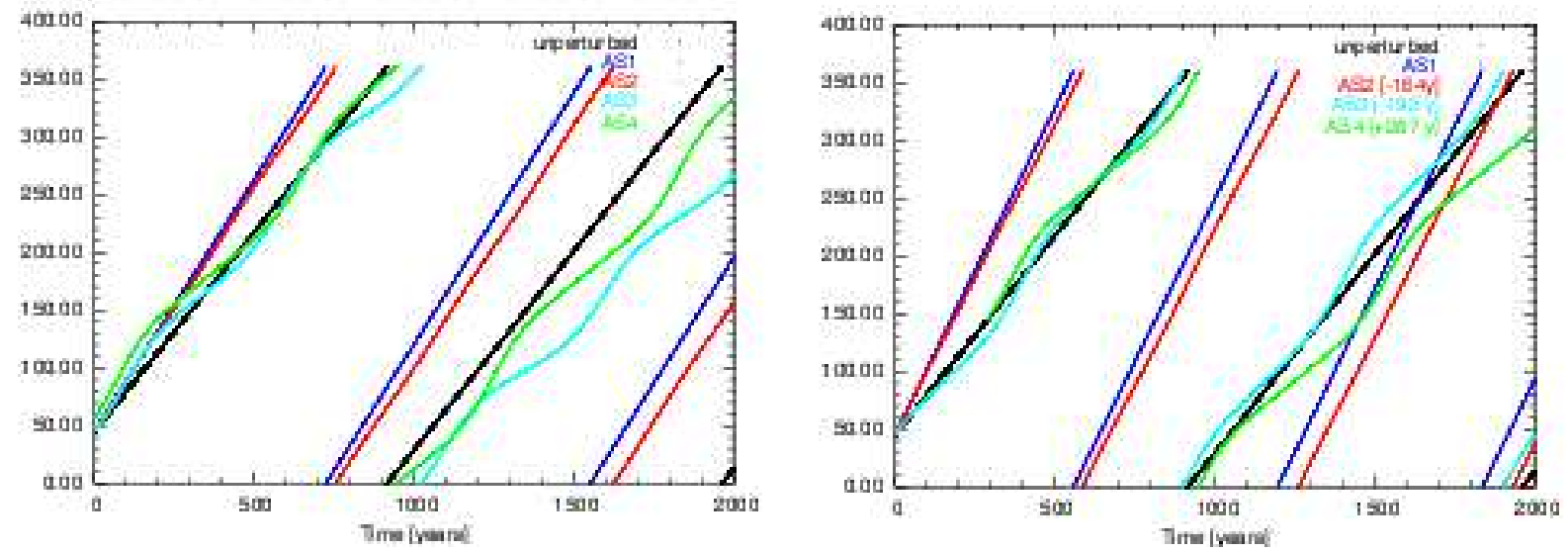

Fig. 10. The variation of the argument of periastron in the observational (left panel) and the dynamical (right panel) frame of reference. In the right panel we shifted the individual curves in time to start the $g$-curves from the same initial value.
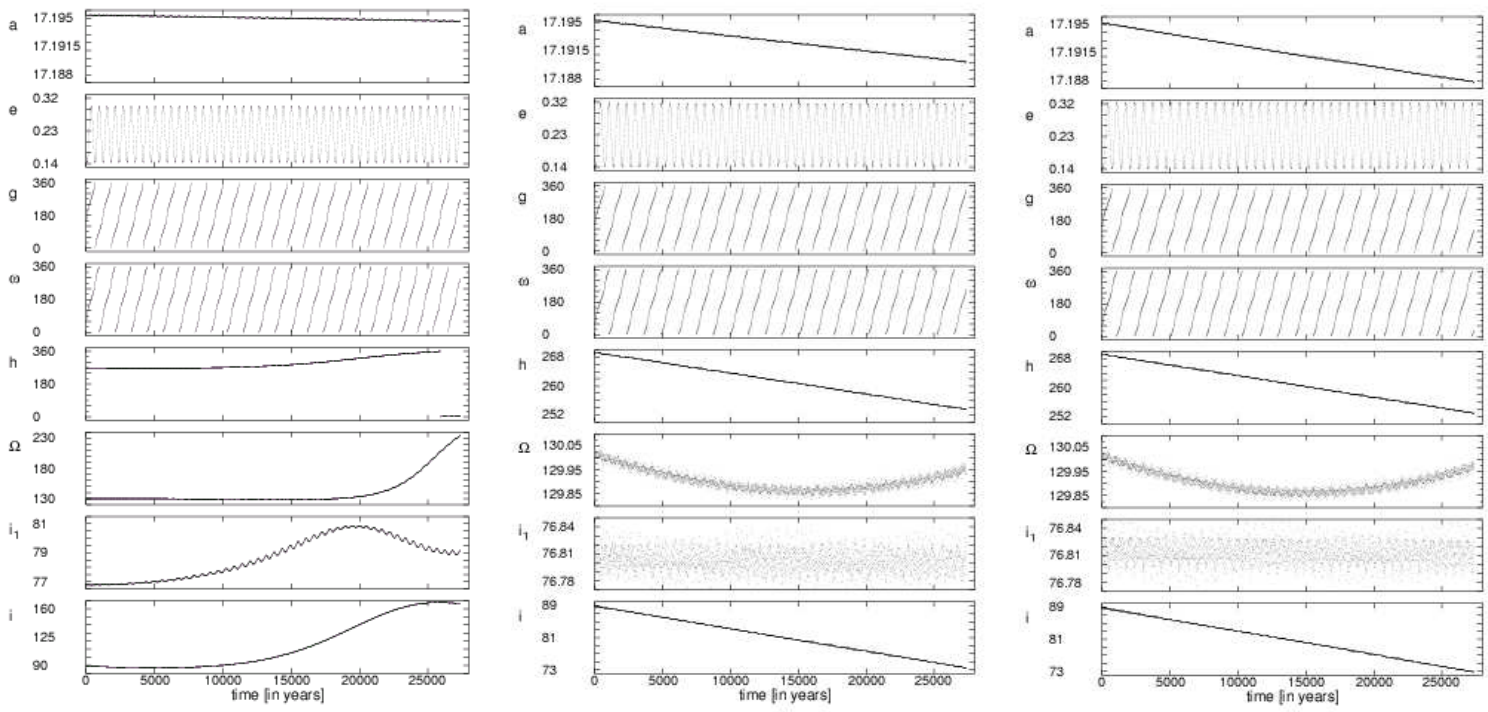

Fig. 11. The variation of the orbital elements of the binary in the dissipative case. The initial parameters are listed in Table 2 Col. AS4. Left: The stellar rotation was initially synchronized in the periastron. Medium: The stellar rotation was synchronized to the average value of the Keplerian angular velocity. Right: The stellar rotation was synchronized in the apastron.

\subsection{Dissipative runs}

Considering the dissipative runs, in Fig.11 we give the variation of the orbital elements in the case of the AS4 configuration for all the three initial synchronizations, i.e. when the synchronization was done to the periastron (left panel), the average value of the orbital angular velocity (medium panel), and the apastron value (right panel). The following differences can be observed.

The shrinkage of the binary orbit is more than three times faster during the total integration time in the apastronsynchronized case. For a qualitative explanation we recall here the simplified dissipative part of the equation of motion of the binary from Eq. (23) of Borkovits et al. (2004). According to this

$\boldsymbol{f}_{\text {dis }} \sim-\lambda_{i}\left(3 \frac{\boldsymbol{\rho}_{1} \cdot \dot{\boldsymbol{\rho}}_{1}}{\rho_{1}^{2}} \boldsymbol{\rho}_{1}-\boldsymbol{p}_{i} \times \boldsymbol{\rho}_{1}\right)$,

where $\rho_{1}$ is the Jacobian vector directed from the primary to the secondary, and $\boldsymbol{p}_{i}$ is the difference vector of the rotational angular velocity vector of the $i$-th star $(i=1,2)$ and the orbital angular velocity vector. As one can see easily, in the apastronsynchronized case the constant $\cdot \boldsymbol{p}_{i} \times \boldsymbol{\rho}_{1}$ terms give a continu- ously negative transversal force component (which has a maximum amplitude at periastron, and almost zero only around the apastron).

The amplitude of the $e$-cycles is also somewhat larger in these latter cases, and the period of the apsidal advance is longer. Nevertheless, the difference in the period between the periastronsynchronized and apastron-synchronized case is about $15 \%$, so it remains clearly under the amount of the observed discrepancy. Furthermore, we note that, although the orbital shrinking as well as the synchronization of the angular rotation are evident on the time-scale of the present integration, it can be clearly seen that there is no evidence for the circularization of the inner orbit. This suggests that the presence of a not so distant third body may basically modify the dissipative circularization process. Naturally, further investigations are needed in this question.

In contradiction to these smaller differences in the behaviour of the above-mentioned orbital elements, the angular elements (both in the observational and the dynamical frames) show completely different variations in these latter two cases than in the periastron-synchronized one. The reason can be found in the special initial configuration, i.e. in the (almost) perpendicular posi- 
tion of the inner and outer orbital plane. In this position the difference in the angular momentum stored initially in the stellar rotation was able to change the direction of the orbital precession.

Finally, we consider the stellar rotation affected by the dissipation. As is well known, the phase space of the rotation of oblate bodies usually contains large chaotic regions (in the sense of our Solar system see e.g. Laskar \& Robutel 1993). In Fig. 12 we show some interesting resonances. We found in several different runs, that when the angular velocity of the rotation of one of the stars becomes temporarily equal to the average orbital angular velocity of the binary, typical resonance phenomena occur, i.e. the amplitude of the stellar precession suddenly increases, or even some fluctuations arise in the stellar rotational rate, and, consequently, this can manifest even in some similar fluctuations in the binary's semi-major axis, as happens in our AS3b run. Detailed investigations of such resonance phenomena may be the subject of future studies.

\section{Discussion and conclusion}

In this paper we gave analytically the net effect of the perturbations of a third companion and the mutual tidal and rotational distortion of the binary members for the orbital elements of an eccentric binary star. We investigated primarily how the presence of the third body can affect the $\mathrm{O}-\mathrm{C}$ curve, which is the most important source of the apsidal motion information in relativistic eccentric eclipsing binaries. For this purpose we have chosen one particular eclipsing system, namely AS Camelopardalis. Nevertheless, naturally, most of our results are valid in general, independently of this specific system, although we stress, that in the case of other systems, or even in the same system with different third body parameters, the magnitude of some parameters can differ from our assumptions.

Our work on this topic is not the first, but one of its main novelties is that we calculated simultaneously the effect of the tidal distortion and the tertiary perturbations. There is a strong interaction between the two physical processes. Here we refer to the strong dependence of the tidal forces on the eccentricity. Consequently, the third body forced eccentricity variation influences notably the tidal phenomena, and besides the pure, third star forced apsidal motion rate variation, this effect produces further remarkable change in the apsidal motion speed. At this point we emphasize again the necessity of the common treatment of the two different physical processes, as their simultaneous presence can basically change the evolution of a close binary system. We mainly refer to the so-called Kozai resonance, but further consideration of this topic can be found in Eggleton et al. (1998). Note that from our Eq. (26) naturally almost the same condition for the tidal prevention of the Kozai resonance arise ${ }^{3}$, which was given in Khodykin et al. (2004) by Eq. (38).

The other novelty, as we already emphasized, is the calculation the mathematical shape of the $\mathrm{O}-\mathrm{C}$ curve, and we thoroughly investigated the reliability of the data which can be obtained from such a distorted $\mathrm{O}-\mathrm{C}$ when only a small portion of the total apsidal motion period is covered.

\footnotetext{
3 We said "almost", because it is not clear for us why the authors use in their analytical stability investigation such orbital elements which refer to the observable system. It should be clear that there is no connection between the physical instability in the system and its orientation with respect to the Earth. So in their Eq. (37) which defines the stability criterium they should use (according to their notation) $\phi$ (this is $-g$ in the present paper), instead of $\omega$.
}

We found that there is a significant domain of the possible hierarchical triple body system configurations where both the dynamical and the observational effects appear corresponding the tendency to measure a longer, and even remarkably longer apsidal advance rate, than is theoretically expected according to the known physical parameters, and the measured eccentricity and visible argument of periastron. This happens when the mutual inclination of the close and the wide orbits is large, i.e. the orbits are nearly perpendicular to each other, and, furthermore, the orbital plane of the tertiary almost coincides with the plane of the sky. As the observable inclination of the anomalously slow apsidal motion eclipsing binaries is necessarily close to $i=90^{\circ}$, this means that almost every second near a perpendicular triple system might belong to the ideal case. Note, that the first condition which mainly (but not exclusively) comes from the dynamical calculations is in accordance with the results of the previously mentioned studies. However, we have shown that it has a notably lower significance alone as stated previously. So, we concluded, that the observational effects can play a remarkably more important role in the detection of anomalously slow apsidal motion rate than the dynamical ones.

This result is all the more impressive, because it offers a natural answer for a further serious question: why have we not observed these third companions yet? We consider this question in detail. Several direct and indirect methods can be used now to detect a not so distant tertiary in a binary system. A relatively detailed, but not complete listing of these methods is found in Pribulla \& Rucinski (2006). However, despite the diversity of the methods, most were applied only for a few binary systems, and we do not know that any of the direct methods would have been applied for any anomalously slow apsidal motion system. So we mainly concentrate here on the most usual, perhaps we can say: "classical", although in several cases very uncertain way of the detection of a third component in an eclipsing binary system, the method of which is based on the light-time effect (LITE) manifesting on the $\mathrm{O}-\mathrm{C}$ diagram. However, the light-time effect arises from the varying distance of the eclipsing system from the observer, due to the revolution of the binary around the centre of mass of the triple system. Consequently, when this motion takes place nearly in the plane of the sky, the distance of the eclipsing binary remains almost constant, so the amplitude of the LITE (related to $a^{\prime} \sin i^{\prime}$ ) might remain under the detection limit. (The same can be said about the detection via the systematic radial velocity variation.) Unfortunately, this is exactly the situation in our ideal case for miscalculating the real apsidal motion period from a small fraction of a complete revolution.

At this point we have to stop for a short interplay, and note, that in the case of AS Camelopardalis, Kozyreva et al. (1999) really reported that the $\mathrm{O}-\mathrm{C}$ curve shows small amplitude periodic variations after the removal of the apsidal motion terms. They fitted light-time solution, and found a third body of mass $\left(m_{3}=1.1-1.7 \mathrm{M}_{\odot}\right)$ orbiting in an eccentric $\left(e^{\prime}=0.5\right)$ orbit with period $P^{\prime}=805 \mathrm{~d}$. However, we also carried out the analysis of the $\mathrm{O}-\mathrm{C}$ diagram, but only a very poor, and consequently, very questionable fit was found (see Borkovits 2003). Nevertheless, we used our calculated orbital elements as input parameters in our numerical studies.

The question naturally arises of what could be an efficient method to detect perturbing bodies (if they exist at all) in such configurations. There are several methods listed in the above mentioned paper of Pribulla \& Rucinski (2006) which could be satisfactory for this purpose. Instead of repeating them, we would like to suggest a further dynamical method, which is based on the direct detection of the perturbations of the ter- 

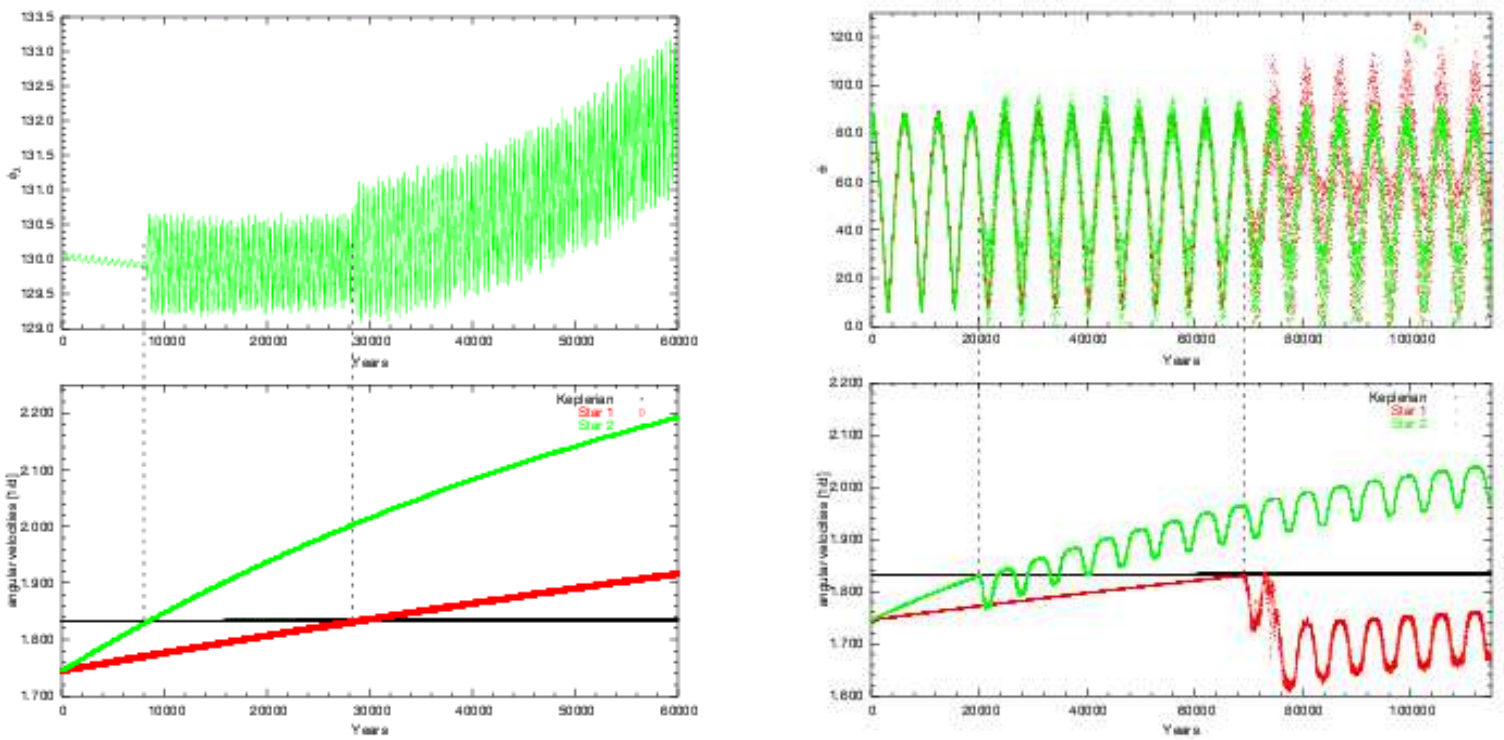

Fig. 12. Resonance phenomena between the orbital revolution and the stellar rotation. When the stellar rotation rate reaches the average orbital revolution rate, the amplitude of the precession of the stellar rotational axes suddenly grows. This figure shows the dissipative AS4 (left) and AS3b (right) runs where the rotational angular velocities initially were set to close to the averaged orbital one.

tiary. As one can see from our results, the variation of the eccentricity $e$ of the binary may reach even some $10^{-2}$ during some decades in our sample system. Such a variation could have been detected by the present accuracy of radial velocity measurements. This is why we suppose that the different values of the eccentricity obtained for AS Cam from the 19691971 and 1981 observations reflect real variation instead of simple observational errors. Note when Maloney et al. 1991 analyzed the 1969-1971 observations of Hilditch (1972a b) and Padalia \& Srivastava (1975), they found that the orbital eccentricity of the binary was $e \approx 0.14$, while Khaliullin \& Kozyreva (1983) deduced from the December 1981 photometry already $e \approx 0.16$. Furthermore, according to recent $\mathrm{O}-\mathrm{C}$ studies (which were cited earlier) $\omega \approx 220^{\circ}-240^{\circ}$ were found. If we assume that the third body revolves close to the plane of the sky, the dynamical argument of periastron $(g)$ should have a similar value. Then, according to our Eq. (54), $\Delta e$ is positive, which is also in good correspondence with the measured tendency. In conclusion, we stress, that it is very important to obtain new precise light curves, as well as radial velocity measurements from this, and other anomalously slow apsidal motion systems. Moreover, as we predict the motion of the tertiary in the plane of the sky, we can expect positive results mainly from the newest interferometric equipments in the near future.

Finally, we also obtained new numerical results on the interaction of the orbital evolution and stellar rotation in close hierarchical triple stellar systems containing an eccentric binary. The most important fact is that resonances might occur due to the variation of the stellar rotational rate during the dissipationdriven synchronization process. Such resonances were found for example in the case when the rotational rate of one of the stars reached the average Keplerian angular velocity of the orbital revolution. It is necessary to investigate these phenomena further in detail.

Acknowledgements. This work was supported in part by the French-Hungarian bilateral project Balaton, Grant No. F36/04. The authors would like to thank Sz. Csizmadia and A. Pál for their valuable comments and suggestions, and Rita Borkovits-József and L. Szabados for the linguistic corrections. This research has made use of NASA's Astrophysics Data System Bibliographic Services.

\section{References}

Borkovits, T., Csizmadia, Sz., Hegedüs, T. et al., 2002, A\&A, 392, 895 Borkovits, T., 2003, Proc. of the "3rd Austrian-Hungarian Workshop on Trojans and Related Topics", held at Vienna, 2002 May 13-15th, (Freistetter, F., Dvorak, R., Érdi, B. eds.), p. 161

Borkovits, T., Érdi, B., Forgács-Dajka, E., \& Kovács, T., 2003, A\&A, 398, 1091

Borkovits, T., Forgács-Dajka, E., \& Regály, Zs., 2004, A\&A, 2004, A\&A, 426, 951

Claret, A., 1998, A\&A, 330, 533

Claret, A., \& Gimènez, A., 1991, A\&AS, 91, 217

Company, R., Portilla, M., \& Gimènez, A., 1988, ApJ, 335, 962

Eggleton, P. P., Kiseleva, L. G., \& Hut, P., 1998, ApJ, 499, 853

Gimènez, A., \& Garcia-Pelayo, J.M., 1983, Ap\&SS, 92, 203

Hegedüs, T., \& Nuspl, J., 1986, Acta Astron., 36, 381

Hilditch, R.W., 1972a, MemRAS, 76, 141

Hilditch, R.W., 1972b, PASP, 84, 519

Khaliullin, Kh., F., \& Kozyreva, V.S., 1983, Ap\&SS, 94, 115

Khaliullin, Kh.F., Khodykin, S.A., \& Zakharov, A.I., 1991, ApJ, 375, 314

Khodykin, S.A., 1989, Astron. Tsirk. 1536

Khodykin, S.A., \& Vedeneyev, V.G., 1997, ApJ, 475, 798

Khodykin, S.A., Zakharov, A.I., \& Andersen, W.L., 2004, ApJ, 615, 506

Kozai, Y., 1962, AJ, 67, 591

Kozyreva, V.S., Zakharov, A.I., \& Khaliullin, Kh.F., 1999, IBVS, No. 4690

Laskar, J., \& Robutel, P., 1993, Nature, 361, 608

Maloney, F.P., Guinan, E.F., \& Boyd, P.T., 1989, AJ, 98, 1800

Maloney, F.P., Guinan, E.F., \& Mukherjee, J., 1991, AJ, 102, 256

Padalia, T.D., \& Srivastava, R.K., 1975, Ap\&SS, 38, 87

Pribulla, T., \& Rucinski, S.M., 2006, AJ, 131, 2986

Semeniuk, T., 1968, Acta Astr., 18, 1

Shakura, N.I., 1985, Sov. Astr. Lett., 11, 224

Söderhjelm, S., 1984, A\&A, 141, 232

Zahn, J.-P., 1977, A\&A, 57, 383 


\section{Appendix A: Calculation of higher order perturbative terms}

As mentioned in the text, in the most interesting cases the amplitude of the eccentricity variation on the apse-node time scale reaches the unity. Consequently, the eccentricity no longer can be considered as constant on the rhs of the perturbation equations. Consequently, first the eccentricity $e$ should be calculated as a function of $g$ in an iterative manner.

The corresponding perturbation equation can be written in the following Taylor-expansion:

$$
\begin{aligned}
\frac{\mathrm{d} e}{\mathrm{~d} g}= & \left(\frac{\mathrm{d} e}{\mathrm{~d} g}\right)_{e_{0}}+\left(\frac{\partial}{\partial e} \frac{\mathrm{d} e}{\mathrm{~d} g}\right)_{e_{0}} \Delta e+\frac{1}{2}\left(\frac{\partial^{2}}{\partial e^{2}} \frac{\mathrm{d} e}{\mathrm{~d} g}\right)_{e_{0}}(\Delta e)^{2}+\ldots \\
= & \left\{[\mathcal{E}]_{0} \Sigma_{0}+\left\{\left[\mathcal{E}+B_{1}+N_{1}-\mathcal{E} A_{1}\right] \Sigma_{0}+\left[\mathcal{E}\left(B_{1}-E A_{1}\right)\right] \Sigma_{1}\right\}_{0} \frac{1}{e_{0}} \Delta e\right. \\
& +\frac{1}{2}\left\{\left[\left(2-2 A_{1}\right)\left(B_{1}+N_{1}-\mathcal{E} A_{1}\right)+B_{2}+N_{2}-\mathcal{E} A_{2}\right] \Sigma_{0}\right. \\
& +\left[2\left(B_{1}+N_{1}\right)\left(B_{1}-E A_{1}\right)+\mathcal{E}\left(B 2-E A_{2}+2 B_{1}-2 E A_{1}-4 A_{1} B_{1}+4 E A_{1}^{2}\right)\right] \Sigma_{1} \\
& \left.\left.+\frac{1}{2}\left[\mathcal{E}\left(B_{1}^{2}+E^{2} A_{1}^{2}-2 E A_{1} B_{1}\right)\right] \Sigma_{2}\right\} \frac{1}{e_{0}^{2}}(\Delta e)^{2}\right\},
\end{aligned}
$$

where

$$
\begin{aligned}
E & =\frac{B}{A}, \\
\mathcal{E} & =\frac{A_{\mathrm{t}}}{A}, \\
A_{1} & =e \frac{1}{A} \frac{\mathrm{d} A}{\mathrm{~d} e}, \\
A_{2} & =e^{2} \frac{1}{A} \frac{\mathrm{d}^{2} A}{\mathrm{~d} e^{2}}, \\
B_{1} & =e \frac{1}{A} \frac{\mathrm{d} B}{\mathrm{~d} e}, \\
B_{2} & =e^{2} \frac{1}{A} \frac{\mathrm{d}^{2} B}{\mathrm{~d} e^{2}}, \\
N_{1} & =e \frac{1}{A} \frac{\mathrm{d} A_{\mathrm{n} 2}}{\mathrm{~d} e}, \\
N_{2} & =e^{2} \frac{1}{A} \frac{\mathrm{d}^{2} A_{\mathrm{n} 2}}{\mathrm{~d} e^{2}}, \\
\Sigma_{0} & =\frac{\sin 2 g}{1+E \cos 2 g}, \\
\Sigma_{1} & =\frac{\mathrm{d} \Sigma_{0}}{\mathrm{~d} E}, \\
\Sigma_{2} & =\frac{\mathrm{d}^{2} \Sigma_{0}}{\mathrm{~d} E^{2}} .
\end{aligned}
$$

(In the calculation of the derivatives above, it should also be considered, that, as it can be seen easily

$$
\begin{aligned}
\frac{\mathrm{d} I}{\mathrm{~d} e} & =\frac{e}{1-e^{2}}\left(I+\frac{C_{1}}{C_{2}}\right), \\
\frac{\mathrm{d} C_{1}}{\mathrm{~d} e} & =-\frac{e}{1-e^{2}} C_{1}, \\
\frac{\mathrm{d} C_{2}}{\mathrm{~d} e} & =0,
\end{aligned}
$$

as far as the present approximation is used.) As the tidal terms depend on the fifth power of the separation, and, consequently, this produces a very strong eccentricity dependence, the $A_{1}, A_{2}$ derivatives may produce some numerical problems even in the case of medium eccentricities. Nevertheless, in the present situation they have the same order of magnitude as $E$ or $\mathcal{E}$, at least at the near-perpendicular configurations. Carrying out the iterative integrations, and writing $e$ into the following Fourier-series

$e=e_{0}\left(1+\epsilon_{0}+\epsilon_{2} \cos 2 g+\epsilon_{4} \cos 4 g+\epsilon_{6} \cos 6 g+\epsilon_{8} \cos 8 g\right)+\ldots$

the coefficients up to fifth order in $E, \mathcal{E}, A_{1}, A_{2}$ are as follows:

$\epsilon_{0}=\left(\frac{1}{8}-\frac{1}{8} A_{1}\right) \mathcal{E}^{2}+\left(\frac{3}{512}-\frac{33}{512} A_{1}-\frac{3}{128} A_{2}\right) \mathcal{E}^{4}+\left(-\frac{9}{256} A_{1}-\frac{3}{256} A_{2}\right) E \mathcal{E}^{3}+\left(\frac{9}{128}-\frac{9}{64} A_{1}\right) E^{2} \mathcal{E}^{2}$ 


$$
\begin{aligned}
& +\left(\frac{1}{8} B_{1}+\frac{1}{8} N_{1}\right) \mathcal{E} \\
& +\left[\frac{1}{2}+\left(\frac{3}{64}-\frac{3}{16} A_{1}-\frac{3}{64} A_{2}+\frac{9}{64} A_{1}^{2}\right) \mathcal{E}^{2}-\frac{1}{32} E \mathcal{E}+\frac{1}{8} E^{2}+\frac{5}{3072} \mathcal{E}^{4}-\frac{1}{768} E \mathcal{E}^{3}+\frac{31}{768} E^{2} \mathcal{E}^{2}-\frac{5}{192} E^{3} \mathcal{E}+\frac{1}{16} E^{4}\right. \\
& \left.+\left(\frac{7}{32} B_{1}+\frac{3}{16} N_{1}+\frac{3}{64} B_{2}+\frac{3}{64} N_{2}\right) \mathcal{E}-\frac{1}{32}\left(B_{1}+N_{1}\right) E\right] \mathcal{E} \cos 2 g_{0} \\
& +\left[\left(\frac{1}{16}-\frac{1}{16} A_{1}\right) \mathcal{E}-\frac{1}{8} E+\left(\frac{1}{192}-\frac{11}{192} A_{1}-\frac{1}{48} A_{2}\right) \mathcal{E}^{3}+\left(-\frac{1}{64}+\frac{5}{96} A_{1}+\frac{1}{96} A_{2}\right) E \mathcal{E}^{2}+\left(\frac{1}{24}-\frac{1}{16} A_{1}\right) E^{2} \mathcal{E}-\frac{1}{16} E^{3}\right. \\
& \left.+\frac{1}{16}\left(B_{1}+N_{1}\right)\right] \mathcal{E} \cos 4 g_{0} \\
& +\left[\left(\frac{1}{192}-\frac{1}{48} A_{1}-\frac{1}{192} A_{2}+\frac{1}{64} A_{1}^{2}\right) \mathcal{E}^{2}+\left(-\frac{1}{32}+\frac{1}{24} A_{1}\right) E \mathcal{E}+\frac{1}{24} E^{2}+\frac{5}{12288} \mathcal{E}^{4}-\frac{3}{1024} E \mathcal{E}^{3}+\frac{31}{3072} E^{2} \mathcal{E}^{2}\right. \\
& \left.-\frac{7}{256} E^{3} \mathcal{E}+\frac{1}{32} E^{4}+\left(\frac{1}{96} B_{1}+\frac{1}{48} N_{1}+\frac{1}{192} B_{2}+\frac{1}{192} N_{2}\right) \mathcal{E}-\frac{1}{32}\left(B_{1}+N_{1}\right) E\right] \mathcal{E} \cos 6 g_{0} \\
& +\left[\left(\frac{1}{3072}-\frac{11}{3072} A_{1}-\frac{1}{768} A_{2}\right) \mathcal{E}^{3}+\left(-\frac{1}{256}+\frac{29}{1536} A_{1}+\frac{7}{1536} A_{2}\right) E \mathcal{E}^{2}+\left(\frac{11}{768}-\frac{3}{128} A_{1}\right) E^{2} \mathcal{E}-\frac{1}{64} E^{3}\right] \mathcal{E} \cos 8 g_{0} \\
& +\left[\frac{1}{61440} \mathcal{E}^{4}-\frac{1}{3072} E \mathcal{E}^{3}+\frac{7}{3072} E^{2} \mathcal{E}^{2}-\frac{5}{768} E^{3} \mathcal{E}+\frac{1}{160} E^{4}\right] \mathcal{E} \cos 10 g_{0}, \\
& \epsilon_{2}=-\frac{1}{2} \mathcal{E}+\left(\frac{1}{64}+\frac{1}{64} A_{2}-\frac{1}{64} A_{1}^{2}\right) \mathcal{E}^{3}+\left(-\frac{1}{32}+\frac{3}{32} A_{1}\right) E \mathcal{E}^{2}-\frac{1}{8} E^{2} \mathcal{E}-\frac{1}{1536} \mathcal{E}^{5}+\frac{1}{384} E \mathcal{E}^{4}+\frac{1}{96} E^{2} \mathcal{E}^{3}-\frac{5}{192} E^{3} \mathcal{E}^{2}-\frac{1}{16} E^{4} \mathcal{E} \\
& -\frac{1}{64}\left(4 B_{1}+B_{2}+N_{2}\right) \mathcal{E}^{2}-\frac{1}{32}\left(B_{1}+N_{1}\right) E \mathcal{E} \\
& +\left[\left(-\frac{1}{2}+\frac{1}{2} A_{1}\right) \mathcal{E}+\left(\frac{1}{64}-\frac{3}{64} A_{1}\right) \mathcal{E}^{3}+\left(-\frac{1}{32}+\frac{9}{32} A_{1}+\frac{3}{32} A_{2}\right) E \mathcal{E}^{2}+\left(-\frac{1}{8}+\frac{3}{8} A_{1}\right) E^{2} \mathcal{E}-\frac{1}{2}\left(B_{1}+N_{1}\right)\right] \epsilon_{0} \\
& +\left[\left(\frac{1}{2} A_{1}+\frac{1}{4} A_{2}-\frac{1}{2} A_{1}^{2}\right) \mathcal{E}-\frac{1}{2}\left(B_{1}+N_{1}\right)-\frac{1}{4}\left(B_{2}+N_{2}\right)\right] \epsilon_{0}^{2} \\
& \epsilon_{4}=\left(\frac{1}{16}-\frac{1}{16} A_{1}\right) \mathcal{E}^{2}+\frac{1}{8} E \mathcal{E} \\
& +\left(-\frac{1}{384}+\frac{1}{192} A_{1}-\frac{1}{768} A_{2}\right) \mathcal{E}^{4}+\left(-\frac{7}{192} A_{1}-\frac{1}{64} A_{2}\right) E \mathcal{E}^{3}+\left(\frac{1}{24}-\frac{5}{48} A_{1}\right) E^{2} \mathcal{E}^{2}+\frac{1}{16} E^{3} \mathcal{E}+\frac{1}{16}\left(B_{1}+N_{1}\right) \mathcal{E} \\
& +\left[\left(\frac{1}{16}-\frac{1}{4} A_{1}-\frac{1}{16} A_{2}+\frac{3}{16} A_{1}^{2}\right) \mathcal{E}^{2}+\left(\frac{1}{8}-\frac{1}{4} A_{1}\right) E \mathcal{E}-\frac{1}{384} \mathcal{E}^{4}+\frac{1}{24} E^{2} \mathcal{E}^{2}+\frac{1}{16} E^{3} \mathcal{E}\right. \\
& \left.+\frac{1}{16}\left(6 B_{1}+4 N_{1}+B_{2}+N_{2}\right) \mathcal{E}+\frac{1}{8}\left(B_{1}+N_{1}\right) E\right] \epsilon_{0}+\left[\left(-\frac{3}{16} A_{1}-\frac{3}{32} A_{2}\right) \mathcal{E}^{2}+\left(-\frac{1}{4} A_{1}-\frac{1}{8} A_{2}\right) E \mathcal{E}\right] \epsilon_{0}^{2}, \\
& \epsilon_{6}=\left(-\frac{1}{192}+\frac{1}{48} A_{1}+\frac{1}{192} A_{2}-\frac{1}{64} A_{1}^{2}\right) \mathcal{E}^{3}+\left(-\frac{1}{32}+\frac{5}{96} A_{1}\right) E \mathcal{E}^{2}-\frac{1}{24} E^{2} \mathcal{E} \\
& +\frac{1}{4096} \mathcal{E}^{5}+\frac{1}{1024} E^{4} \mathcal{E}-\frac{5}{1024} E^{2} \mathcal{E}^{3}-\frac{7}{256} E^{3} \mathcal{E}^{2}-\frac{1}{32} E^{4} \mathcal{E}-\frac{1}{192}\left(8 B_{1}+4 N_{1}+B_{2}+N_{2}\right) \mathcal{E}^{2}-\frac{1}{32}\left(B_{1}+N_{1}\right) E \mathcal{E} \\
& +\left[\left(-\frac{1}{192}+\frac{11}{192} A_{1}+\frac{1}{48} A_{2}\right) \mathcal{E}^{3}+\left(-\frac{1}{32}+\frac{19}{96} A_{1}+\frac{5}{96} A_{2}\right) E \mathcal{E}^{2}+\left(-\frac{1}{24}+\frac{1}{8} A_{1}\right) E^{2} \mathcal{E}\right] \epsilon_{0}, \\
& \epsilon_{8}=\left(\frac{1}{3072}-\frac{11}{3072} A_{1}-\frac{1}{768} A_{2}\right) \mathcal{E}^{4}+\left(\frac{1}{256}-\frac{35}{1536} A_{1}-\frac{3}{512} A_{2}\right) \mathcal{E}^{3} E+\left(\frac{11}{768}-\frac{13}{384} A_{1}\right) E^{2} \mathcal{E}^{2}+\frac{1}{64} E^{3} \mathcal{E} \\
& +\left(\frac{1}{3072} \mathcal{E}^{4}+\frac{1}{256} E \mathcal{E}^{3}+\frac{11}{768} E^{2} \mathcal{E}^{2}+\frac{1}{64} E^{3} \mathcal{E}\right) \epsilon_{0}, \\
& \epsilon_{10}=-\frac{1}{61440} \mathcal{E}^{5}-\frac{1}{3072} E \mathcal{E}^{4}-\frac{7}{3072} E^{2} \mathcal{E}^{3}-\frac{5}{768} E^{3} \mathcal{E}^{2}-\frac{1}{160} E^{4} \mathcal{E}
\end{aligned}
$$


For $\frac{\mathrm{d} u}{\mathrm{~d} g}$ a similar equation can be written as Eq. (A.2) and this gives the $u(g)$ relation as follows:

$\Pi^{*}\left(u-u_{0}^{*}\right)=g+\gamma_{2} \sin 2 g+\gamma_{4} \sin 4 g+\gamma_{6} \sin 6 g+\gamma_{8} \sin 8 g+\gamma_{10} \sin 10 g+\ldots$

where $\left(\Pi^{*}\right)^{-1}$ gives the apsidal motion period in the invariable plane in the unit of the inner orbital period, i.e.

$$
\begin{aligned}
\left(\Pi^{*}\right)^{-1}= & \Pi^{-1}\left\{1+\left(-\frac{1}{16} A_{2}+\frac{1}{8} A_{1}^{2}\right) \mathcal{E}^{2}-\frac{1}{2} A_{1} E \mathcal{E}+\left(\frac{3}{1024} A_{2}-\frac{3}{512} A_{1}^{2}+\frac{1}{512} A_{1} A_{2}+\frac{1}{256} A_{2}^{2}\right) \mathcal{E}^{4}\right. \\
& +\left(\frac{1}{64} A_{1}-\frac{7}{256} A_{2}+\frac{9}{128} A_{1}^{2}+\frac{15}{256} A_{1} A_{2}\right) E \mathcal{E}^{3}+\left(-\frac{5}{64} A_{1}-\frac{45}{256} A_{2}+\frac{103}{128} A_{1}^{2}\right) E^{2} \mathcal{E}^{2}-\frac{23}{32} A_{1} E^{3} \mathcal{E} \\
& +\frac{1}{4} B_{1} \mathcal{E}+\frac{1}{128}\left(-B_{1}+B_{2}\right) \mathcal{E}^{3}+\left(\frac{3}{64} B_{1}+\frac{7}{64} B_{2}\right) E \mathcal{E}^{2}+\frac{9}{16} B_{1} E^{2} \mathcal{E} \\
& +\left[-A_{1}+\left(-\frac{1}{8} A_{2}+\frac{1}{4} A_{1}^{2}+\frac{1}{8} A_{1} A_{2}-\frac{1}{4} A_{1}^{3}\right) \mathcal{E}^{2}+\left(-\frac{1}{2} A_{1}-\frac{1}{2} A_{2}+2 A_{1}^{2}\right) E \mathcal{E}-A_{1} E^{2}+\frac{3}{512} A_{2} \mathcal{E}^{4}\right. \\
& +\left(\frac{1}{64} A_{1}-\frac{5}{128} A_{2}\right) E \mathcal{E}^{3}+\left(-\frac{5}{64} A_{1}-\frac{55}{128} A_{2}\right) E^{2} \mathcal{E}^{2}-\frac{23}{32}\left(A_{1}+A_{2}\right) E^{3} \mathcal{E}-A_{1} E^{4} \\
& \left.+\left(\frac{1}{4}\left(B_{1}+B_{2}\right)-\frac{5}{4} A_{1} B_{1}-\frac{1}{8} A_{2}\left(B_{1}+N_{1}\right)\right) \mathcal{E}+\left(B_{1}-\frac{1}{2} A_{1}\left(B_{1}+N_{1}\right)\right) E\right] \epsilon_{0} \\
& +\left[-\frac{1}{2} A_{2}+A_{1}^{2}+\left(-\frac{1}{16} A_{2}+\frac{1}{8} A_{1}^{2}+\frac{1}{4} A_{1} A_{2}+\frac{1}{16} A_{2}^{2}\right) \mathcal{E}^{2}+\left(-\frac{1}{2} A_{2}+2 A_{1}^{2}+\frac{3}{4} A_{1} A_{2}\right) E \mathcal{E}+\left(-\frac{1}{2} A_{2}+\frac{5}{2} A_{1}^{2}\right) E^{2}\right. \\
& \left.\left.+\frac{1}{4} B_{2} \mathcal{E}+\frac{1}{2} B_{2} E\right] \epsilon_{0}^{2}\right\},
\end{aligned}
$$

where as before

$\Pi=A \sqrt{1-E^{2}}$.

Furthermore,

$$
\begin{aligned}
& \gamma_{2}=-\frac{1}{2} E-\frac{1}{8} E^{3}-\frac{1}{16} E^{5} \\
& +\frac{1}{4} A_{1} \mathcal{E}-\frac{1}{128}\left(A_{1}-A_{2}+2 A_{1}^{2}\right) \mathcal{E}^{3}+\frac{1}{64}\left(3 A_{1}+5 A_{2}-13 A_{1}^{2}\right) E \mathcal{E}^{2}+\frac{5}{16} A_{1} E^{2} \mathcal{E}-\frac{1}{64}\left(B_{1}+3 B_{2}\right) \mathcal{E}^{2}-\frac{9}{32} B_{1} E \mathcal{E} \\
& +\left[\frac{1}{4}\left(A_{1}+A_{2}-2 A_{1}^{2}\right) \mathcal{E}-\frac{1}{128}\left(A_{1}-A_{2}\right) \mathcal{E}^{3}+\frac{1}{64}\left(3 A_{1}+13 A_{2}\right) E \mathcal{E}^{2}+\frac{5}{16}\left(A_{1}+A_{2}\right) E^{2} \mathcal{E}+\frac{3}{8} A_{1} E^{3}\right. \\
& \left.-\frac{1}{2} B_{1}+\frac{1}{4} A_{1}\left(B_{1}+N_{1}\right)\right] \epsilon_{0}+\left[\frac{1}{4}\left(A_{2}-2 A_{1}^{2}\right) \mathcal{E}+\frac{1}{4}\left(A_{2}-2 A_{1}^{2}\right) E-\frac{1}{4} B_{2}\right] \epsilon_{0}^{2}, \\
& \gamma_{4}=\frac{1}{8} E^{2}+\frac{1}{16} E^{4} \\
& -\frac{1}{64}\left(A_{1}+A_{2}+3 A_{1}^{2}\right) \mathcal{E}^{2}-\frac{5}{32} A_{1} E \mathcal{E}+\frac{1}{1536}\left(A_{1}+A_{2}\right) \mathcal{E}^{4}+\frac{1}{768}\left(2 A_{1}-9 A_{2}\right) E \mathcal{E}^{3}-\frac{1}{192}\left(8 A_{1}+11 A_{2}\right) E^{2} \mathcal{E}^{2}-\frac{41}{192} A_{1} E^{3} \mathcal{E} \\
& +\left(\frac{1}{16} B_{1}-\frac{1}{64} A_{1}\left(B_{1}+N_{1}\right)\right) \mathcal{E} \\
& +\left[-\frac{1}{64}\left(A_{1}+3 A_{2}+9 A_{1}^{2}+3 A_{1} A_{2}\right) \mathcal{E}^{2}-\frac{5}{32}\left(A_{1}+A_{2}-3 A_{1}^{2}\right) E \mathcal{E}-\frac{1}{4} A_{1} E^{2}+\frac{1}{16}\left(B_{1}+B_{2}\right) \mathcal{E}+\frac{1}{4} B_{1} E\right] \epsilon_{0} \\
& +\left[-\frac{1}{32} A_{2} \mathcal{E}^{2}-\frac{5}{32} A_{2} E \mathcal{E}-\frac{1}{8} A_{2} E^{2}\right] \epsilon_{0}^{2} \\
& \gamma_{6}=-\frac{1}{24} E^{3}-\frac{1}{32} E^{5} \\
& +\frac{1}{1152}\left(A_{1}+3 A_{2}-10 A_{1}^{2}-4 A_{1} A_{2}\right) \mathcal{E}^{3}+\frac{1}{576}\left(9 A_{1}+9 A_{2}-35 A_{1}^{2}\right) E \mathcal{E}^{2}+\frac{13}{144} A_{1} E^{2} \mathcal{E}-\frac{1}{192}\left(B_{1}+B_{2}\right) \mathcal{E}^{2}-\frac{5}{96} B_{1} E \mathcal{E} \\
& +\left[\frac{1}{1152}\left(A_{1}+7 A_{2}\right) \mathcal{E}^{3}+\frac{1}{64}\left(A_{1}+3 A_{2}\right) E \mathcal{E}^{2}+\frac{13}{144}\left(A_{1}+A_{2}\right) E^{2} \mathcal{E}+\frac{1}{8} A_{1} E^{3}\right] \epsilon_{0},
\end{aligned}
$$


$\gamma_{8}=\frac{1}{64} E^{4}-\frac{1}{24576}\left(A_{1}+7 A_{2}\right) \mathcal{E}^{4}-\frac{7}{6144}\left(A_{1}+3 A_{2}\right) E \mathcal{E}^{3}-\frac{71}{6144}\left(A_{1}+A_{2}\right) E^{2} \mathcal{E}^{2}-\frac{77}{1536} A_{1} E^{3} \mathcal{E}$,

$\gamma_{10}=-\frac{1}{160} E^{5}$

and, finally,

$\Pi^{*} u_{0}^{*}=\Pi^{*} u_{0}-\left(g_{0}+\gamma_{2} \sin 2 g_{0}+\gamma_{4} \sin 4 g_{0}+\gamma_{6} \sin 6 g_{0}+\gamma_{8} \sin 8 g_{0}+\gamma_{10} \sin 10 g_{0}\right)+O\left(e^{6}, E^{6}\right)$.

As the next step we carry out the inverse transformation. Introducing the variable

$\mathcal{G}=\Pi^{*}\left(u-u_{0}^{*}\right)$,

the Fourier coefficients of the following equation

$g=\mathcal{G}+G_{2} \sin 2 \mathcal{G}+G_{4} \sin 4 \mathcal{G}+G_{6} \sin 6 \mathcal{G}+G_{8} \sin 8 \mathcal{G}+G_{10} \sin 10 \mathcal{G}+\ldots$

can be calculated as e.g.

$G_{n}=\frac{1}{\pi} \int_{0}^{2 \pi}(g-\mathcal{G}) \sin n \mathcal{G}(g) \frac{\mathrm{d} \mathcal{G}}{\mathrm{d} g} \mathrm{~d} g$,

where both $\sin n \mathcal{G}(g)$ and $\frac{\mathrm{d} \mathcal{G}}{\mathrm{d} g}$ can easily be calculated from Eq. A.24). The individual coefficients are as follows:

$$
\begin{aligned}
G_{2}= & \frac{1}{2} E+\frac{1}{8} E^{3}+\frac{1}{16} E^{5} \\
& -\frac{1}{4} A_{1} \mathcal{E}+\frac{1}{256}\left(2 A_{1}-2 A_{2}+5 A_{1}^{2}+A_{1} A_{2}\right) \mathcal{E}^{3}-\frac{1}{128}\left(7 A_{1}+11 A_{2}-28 A_{1}^{2}\right) E \mathcal{E}^{2}-\frac{21}{64} A_{1} E^{2} \mathcal{E}+\frac{1}{64}\left(B_{1}+3 B_{2}\right) \mathcal{E}^{2}+\frac{5}{16} B_{1} E \mathcal{E} \\
& +\left[-\frac{1}{4}\left(A_{1}+A_{2}-2 A_{1}^{2}\right) \mathcal{E}-\frac{1}{2} A_{1} E+\frac{1}{128}\left(A_{1}-A_{2}\right) \mathcal{E}^{3}-\frac{1}{128}\left(7 A_{1}+29 A_{2}\right) E \mathcal{E}^{2}-\frac{21}{64}\left(A_{1}+A_{2}\right) E^{2} \mathcal{E}-\frac{3}{8} A_{1} E^{3}\right. \\
& \left.+\frac{1}{2} B_{1}-\frac{1}{4} A_{1}\left(B_{1}+N_{1}\right)\right] \epsilon_{0}+\left[-\frac{1}{4}\left(A_{2}-2 A_{1}^{2}\right) \mathcal{E}-\frac{1}{4}\left(A_{2}-2 A_{1}^{2}\right) E+\frac{1}{4} B_{2}\right] \epsilon_{0}^{2}, \\
G_{4}= & \frac{1}{8} E^{2}+\frac{1}{16} E^{4} \\
& +\frac{1}{64}\left(A_{1}+A_{2}+A_{1}^{2}\right) \mathcal{E}^{2}-\frac{3}{32} A_{1} E \mathcal{E}-\frac{1}{1536}\left(A_{1}+A_{2}\right) \mathcal{E}^{4}+\frac{1}{2304}\left(14 A_{1}+15 A_{2}\right) E \mathcal{E}^{3}-\frac{1}{192}\left(A_{1}+4 A_{2}\right) E^{2} \mathcal{E}^{2}-\frac{95}{576} A_{1} E^{3} \mathcal{E} \\
& -\frac{1}{16} B_{1} \mathcal{E}+\frac{1}{64} A_{1}\left(B_{1}+N_{1}\right) \mathcal{E} \\
& +\left[\frac{1}{64}\left(A_{1}+3 A_{2}-A_{1}^{2}+5 A_{1} A_{2}\right) \mathcal{E}^{2}-\frac{3}{32}\left(A_{1}+A_{2}+3 A_{1}^{2}\right) E \mathcal{E}-\frac{1}{4} A_{1} E^{2}-\frac{1}{16}\left(B_{1}+B_{2}\right) \mathcal{E}+\frac{1}{4} B_{1} E\right] \epsilon_{0} \\
& +\left[\frac{1}{32} A_{2} \mathcal{E}^{2}-\frac{3}{32} A_{2} E \mathcal{E}-\frac{1}{8} A_{2} E^{2}\right] \epsilon_{0}^{2}, \\
G_{6}= & \frac{1}{24} E^{3}+\frac{1}{32} E^{5} \\
& -\frac{1}{2304}\left(2 A_{1}+6 A_{2}+7 A_{1}^{2}+19 A_{1} A_{2}\right) \mathcal{E}^{3}+\frac{1}{1152}\left(9 A_{1}+9 A_{2}+16 A_{1}^{2}\right) E \mathcal{E}^{2}-\frac{25}{576} A_{1} E^{2} \mathcal{E}+\frac{1}{192}\left(B_{1}+B_{2}\right) \mathcal{E}^{2}-\frac{1}{24} B_{1} E \mathcal{E} \\
& +\left[-\frac{1}{1152}\left(A_{1}+7 A_{2}\right) \mathcal{E}^{3}+\frac{1}{128}\left(A_{1}+3 A_{2}\right) E \mathcal{E}^{2}-\frac{25}{576}\left(A_{1}+A_{2}\right) E^{2} \mathcal{E}-\frac{1}{8} A_{1} E^{3}\right] \epsilon_{0},
\end{aligned}
$$

$$
\begin{aligned}
G_{8}= & \frac{1}{64} E^{4} \\
& +\frac{1}{24576}\left(A_{1}+7 A_{2}\right) \mathcal{E}^{4}-\frac{11}{18432}\left(A_{1}+3 A_{2}\right) E \mathcal{E}^{3}+\frac{23}{6144}\left(A_{1}+A_{2}\right) E^{2} \mathcal{E}^{2}-\frac{97}{4608} A_{1} E^{3} \mathcal{E},
\end{aligned}
$$

$G_{10}=\frac{1}{160} E^{5}$.

By the use of Eq. A.34 the other orbital elements can also be easily expressed as a function of $u$, namely $e=e_{0}\left(1+E_{00}+E_{2} \cos 2 \mathcal{G}+E_{4} \cos 4 \mathcal{G}+E_{6} \cos 6 \mathcal{G}+E_{8} \cos 8 \mathcal{G}+E_{10} \cos 10 \mathcal{G}\right)+\ldots$, 
where

$$
\begin{aligned}
& E_{00}=-\frac{1}{8} A_{1} \mathcal{E}^{2}+\frac{1}{4} E \mathcal{E}+\frac{3}{512}\left(A_{1}-A_{2}\right) \mathcal{E}^{4}-\frac{1}{256}\left(2+14 A_{1}+13 A_{2}\right) E \mathcal{E}^{3}+\frac{1}{128}\left(4-37 A_{1}\right) E^{2} \mathcal{E}^{2}+\frac{5}{32} E^{3} \mathcal{E} \\
& +\left[1-\frac{1}{8}\left(2 A_{1}+A_{2}-3 A_{1}^{2}\right) \mathcal{E}^{2}+\frac{1}{4}\left(1-2 A_{1}\right) E \mathcal{E}-\frac{1}{128} E \mathcal{E}^{3}+\frac{1}{32} E^{2} \mathcal{E}^{2}+\frac{5}{32} E^{3} \mathcal{E}+\frac{1}{4} B_{1} \mathcal{E}+\frac{1}{4}\left(B_{1}+N_{1}\right) E\right] \epsilon_{0} \\
& +\left[-\frac{1}{8}\left(A_{1}+2 A_{2}\right) \mathcal{E}^{2}-\frac{1}{4}\left(2 A_{1}+A_{2}\right) E \mathcal{E}\right] \epsilon_{0}^{2}, \\
& E_{2}=-\frac{1}{2} \mathcal{E}+\frac{1}{128}\left(2+5 A_{1}+3 A_{2}-3 A_{1}^{2}\right) \mathcal{E}^{3}-\frac{1}{64}\left(6-7 A_{1}\right) E \mathcal{E}^{2}-\frac{1}{8} E^{2} \mathcal{E}-\frac{1}{1536} \mathcal{E}^{5}+\frac{1}{192} E \mathcal{E}^{4}+\frac{1}{384} E^{2} \mathcal{E}^{3}-\frac{1}{12} E^{3} \mathcal{E}^{2} \\
& -\frac{1}{16} E^{4} \mathcal{E}-\frac{1}{64}\left(6 B_{1}+B_{2}+N_{2}\right) \mathcal{E}^{2}-\frac{3}{32}\left(B_{1}+N_{1}\right) E \mathcal{E} \\
& +\left[-\frac{1}{2}\left(1-A_{1}\right) \mathcal{E}+\frac{1}{64}\left(1+2 A_{1}+4 A_{2}\right) \mathcal{E}^{3}-\frac{1}{64}\left(6-32 A_{1}-7 A_{2}\right) E \mathcal{E}^{2}-\frac{1}{8}\left(1-3 A_{1}\right) E^{2} \mathcal{E}-\frac{1}{2}\left(B_{1}+N_{1}\right)\right] \epsilon_{0} \\
& +\left[\frac{1}{4}\left(2 A_{1}+A_{2}-2 A_{1}^{2}\right) \mathcal{E}-\frac{1}{2}\left(B_{1}+N_{1}\right)-\frac{1}{4}\left(B_{2}+N_{2}\right)\right] \epsilon_{0}^{2}, \\
& E_{4}=\frac{1}{16}\left(1+A_{1}\right) \mathcal{E}^{2}-\frac{1}{8} E \mathcal{E}-\frac{1}{2304}\left(6+16 A_{1}-3 A_{2}\right) \mathcal{E}^{4}+\frac{1}{192}\left(3+2 A_{1}+6 A_{2}\right) E \mathcal{E}^{3}+\frac{1}{288}\left(3+38 A_{1}\right) E^{2} \mathcal{E}^{2}-\frac{1}{16} E^{3} \mathcal{E} \\
& +\frac{1}{16}\left(B_{1}+N_{1}\right) \mathcal{E}+\left[\frac{1}{16}\left(1+A_{2}-3 A_{1}^{2}\right) \mathcal{E}^{2}-\frac{1}{8}\left(1-2 A_{1}\right) E \mathcal{E}-\frac{1}{384} \mathcal{E}^{4}+\frac{1}{64} E \mathcal{E}^{3}+\frac{1}{96} E^{2} \mathcal{E}^{2}-\frac{1}{16} E^{3} \mathcal{E}\right. \\
& \left.+\frac{1}{16}\left(2 B_{1}+4 N_{1}+B_{2}+N_{2}\right)-\frac{1}{8}\left(B_{1}+N_{1}\right) E\right] \epsilon_{0}+\left[\frac{1}{32}\left(-2 A_{1}+5 A_{2}\right) \mathcal{E}^{2}+\frac{1}{8}\left(2 A_{1}+A_{2}\right) E \mathcal{E}\right] \epsilon_{0}^{2}, \\
& E_{6}=-\frac{1}{384}\left(2+7 A_{1}+A_{2}+3 A_{1}^{2}\right) \mathcal{E}^{3}+\frac{1}{192}\left(6+7 A_{1}\right) E \mathcal{E}^{2}-\frac{1}{24} E^{2} \mathcal{E}+\frac{1}{4096} \mathcal{E}^{5}-\frac{7}{3072} E \mathcal{E}^{4}+\frac{3}{1024} E^{2} \mathcal{E}^{3}+\frac{13}{768} E^{3} \mathcal{E}^{2} \\
& -\frac{1}{32} E^{4} \mathcal{E}-\frac{1}{192}\left(2 B_{1}+4 N_{1}+B_{2}+N_{2}\right) \mathcal{E}^{2}+\frac{1}{32}\left(B_{1}+N_{1}\right) E \mathcal{E} \\
& +\left[-\frac{1}{192}\left(1+4 A_{1}+8 A_{2}\right) \mathcal{E}^{3}+\frac{1}{192}\left(6-4 A_{1}+7 A_{2}\right) E \mathcal{E}^{2}-\frac{1}{24}\left(1-3 A_{1}\right) E^{2} \mathcal{E}\right] \epsilon_{0}, \\
& E_{8}=\frac{1}{9216}\left(3+25 A_{1}+18 A_{2}\right) \mathcal{E}^{4}-\frac{1}{1536}\left(6+23 A_{1}+3 A_{2}\right) E \mathcal{E}^{3}+\frac{11}{768} E^{2} \mathcal{E}^{2}-\frac{1}{64} E^{3} \mathcal{E} \\
& +\left[\frac{1}{3072} \mathcal{E}^{4}-\frac{1}{256} E \mathcal{E}^{3}+\frac{11}{2304}\left(3+4 A_{1}\right) E^{2} \mathcal{E}^{2}-\frac{1}{64} E^{3} \mathcal{E}\right] \epsilon_{0}, \\
& E_{10}=-\frac{1}{61440} \mathcal{E}^{5}+\frac{1}{3072} E \mathcal{E}^{4}-\frac{7}{3072} E^{2} \mathcal{E}^{3}+\frac{5}{768} E^{3} \mathcal{E}^{2}-\frac{1}{160} E^{4} \mathcal{E}
\end{aligned}
$$

The perturbations of the other orbital elements can be calculated in a similar way, but as our final purpose is to obtain the analytical form of the $\mathrm{O}-\mathrm{C}$ in the function of the eclipsing cycle number (which is highly related to $u$, as well as $\mathcal{G}$ ), now we use the following direct relation,

$\frac{\mathrm{d} X}{\mathrm{~d} \mathcal{G}}=\left(\frac{\mathrm{d} X}{\mathrm{~d} \mathcal{G}}\right)_{e_{0}}+\left(\frac{\partial}{\partial e} \frac{\mathrm{d} X}{\mathrm{~d} \mathcal{G}}\right)_{e_{0}} \Delta e+\frac{1}{2}\left(\frac{\partial^{2}}{\partial e^{2}} \frac{\mathrm{d} X}{\mathrm{~d} \mathcal{G}}\right)_{e_{0}}(\Delta e)^{2}+\ldots$,

where $X$ means any of the remaining orbital elements or related quantities. So, for the node $(h)$ in the dynamical system, as well as for $u_{\mathrm{m} 1}$ (which the latter denotes that part of $u_{\mathrm{m}}$ which can be derived from $\mathrm{d} h \cos i_{1}$ ):

$$
\begin{aligned}
h & =h_{0}^{*}+H_{0} \mathcal{G}+H_{2} \sin 2 \mathcal{G}+H_{4} \sin 4 \mathcal{G}+H_{6} \sin 6 \mathcal{G}+H_{8} \sin 8 \mathcal{G}+\ldots, \\
u_{\mathrm{m} 1} & =\left(u_{\mathrm{m} 1}\right)_{0}^{*}+U_{0} \mathcal{G}+U_{2} \sin 2 \mathcal{G}+U_{4} \sin 4 \mathcal{G}+U_{6} \sin 6 \mathcal{G}+U_{8} \sin 8 \mathcal{G}+\ldots,
\end{aligned}
$$

where

$$
\begin{aligned}
H_{0}= & J+K+\left[\frac{1}{16}\left(K_{2}+J_{2}\right)-\frac{1}{8} A_{1}\left(J_{1}+K_{1}\right)\right] \mathcal{E}^{2}+\frac{1}{4}\left(J_{1}+K_{1}\right) E \mathcal{E} \\
& +\frac{1}{4}\left(-A_{1} K+K_{1}\right) \mathcal{E}+\frac{1}{2} K E-\frac{1}{128}\left(K_{1}-K_{2}\right) \mathcal{E}^{3}+\frac{1}{32}\left(K_{1}+2 K_{2}\right) E \mathcal{E}^{2}+\frac{5}{32} K_{1} E^{2} \mathcal{E}+\frac{1}{8} K E^{3} \\
& +\left[J_{1}+K_{1}+\frac{1}{4}\left(K_{1}+K_{2}-2 A_{1} K_{1}-A_{1} K-A_{2} K\right) \mathcal{E}+\frac{1}{2}\left(K_{1}-A_{1} K\right) E+\frac{1}{8}\left(J_{2}+K_{2}\right) \mathcal{E}^{2}+\frac{1}{4}\left(J_{1}+K_{1}+J_{2}+K_{2}\right) E \mathcal{E}\right] \epsilon_{0} \\
& +\left[\frac{1}{2}\left(J_{2}+K_{2}\right)+\frac{1}{4} K_{2} \mathcal{E}+\frac{1}{4} K_{2} E\right] \epsilon_{0}^{2},
\end{aligned}
$$




$$
\begin{aligned}
H_{2}= & -\frac{1}{2} K+\left[\frac{1}{128}\left(A_{1}+A_{2}\right) K-\frac{1}{64}\left(1-5 A_{1}\right) K_{1}-\frac{3}{64} K_{2}\right] \mathcal{E}^{2}-\left[\frac{7}{64} A_{1} K+\frac{5}{32} K_{1}\right] E \mathcal{E}+\frac{1}{8} K E^{2} \\
& -\frac{1}{4}\left(J_{1}+K_{1}\right) \mathcal{E}+\frac{1}{128}\left(J_{1}+K_{1}-J_{2}-K_{2}\right) \mathcal{E}^{3}-\frac{3}{64}\left(J_{1}+K_{1}+J_{2}+K_{2}\right) E \mathcal{E}^{2}-\frac{1}{16}\left(J_{1}+K_{1}\right) E^{2} \mathcal{E} \\
& +\left[-\frac{1}{2} K_{1}-\frac{1}{4}\left(J_{1}+K_{1}+J_{2}+K_{2}-A_{1} J_{1}-A_{1} K_{1}\right) \mathcal{E}-\frac{1}{64}\left(K_{1}+7 K_{2}\right) \mathcal{E}^{2}-\frac{5}{32}\left(K_{1}+K_{2}\right) E \mathcal{E}+\frac{1}{8} K_{1} E^{2}\right] \epsilon_{0} \\
& +\left[-\frac{1}{4} K_{2}-\frac{1}{4}\left(J_{2}+K_{2}\right) \mathcal{E}\right] \epsilon_{0}^{2},
\end{aligned}
$$

$$
\begin{aligned}
H_{4}= & \frac{1}{16}\left(K_{1}+A_{1} K\right) \mathcal{E}-\frac{1}{8} K E-\frac{1}{768}\left(K_{1}-3 K_{2}\right) \mathcal{E}^{3}+\frac{1}{128}\left(2 K_{1}+K_{2}\right) E \mathcal{E}^{2}-\frac{5}{192} K_{1} E^{2} \mathcal{E} \\
& +\frac{1}{64}\left(J_{1}+K_{1}+J_{2}+K_{2}+A_{1} J_{1}+A_{1} K_{1}\right) \mathcal{E}^{2}-\frac{1}{32}\left(J_{1}+K_{1}\right) E \mathcal{E} \\
& +\left[\frac{1}{16}\left(K_{1}+K_{2}+A_{1} K+A_{2} K\right) \mathcal{E}-\frac{1}{8}\left(K_{1}-A_{1} K\right) E+\frac{1}{64}\left(J_{1}+K_{1}+3 J_{2}+3 K_{2}\right) \mathcal{E}^{2}-\frac{1}{32}\left(J_{1}+K_{1}+J_{2}+K_{2}\right) E \mathcal{E}\right] \epsilon_{0} \\
& +\left[\frac{1}{16} K_{2} \mathcal{E}-\frac{1}{16} K_{2} E\right] \epsilon_{0}^{2},
\end{aligned}
$$$$
H_{6}=-\left[\frac{1}{384}\left(A_{1}+A_{2}\right) K+\frac{1}{192}\left(1+3 A_{1}\right) K_{1}+\frac{1}{192} K_{2}\right] \mathcal{E}^{2}+\frac{1}{192}\left(7 A_{1} K+6 K_{1}\right) E \mathcal{E}-\frac{1}{24} K E^{2}
$$$$
-\frac{1}{1152}\left(J_{1}+K_{1}+3 J_{2}+3 K_{2}\right) \mathcal{E}^{3}+\frac{1}{192}\left(J_{1}+K_{1}+J_{2}+K_{2}\right) E \mathcal{E}^{2}-\frac{1}{144}\left(J_{1}+K_{1}\right) E^{2} \mathcal{E}
$$$$
+\left[-\frac{1}{192}\left(K_{1}+3 K_{2}\right) \mathcal{E}^{2}+\frac{1}{32}\left(K_{1}+K_{2}\right) E \mathcal{E}-\frac{1}{24} K_{1} E^{2}\right] \epsilon_{0},
$$

$H_{8}=\frac{1}{3072}\left(K_{1}+3 K_{2}\right) \mathcal{E}^{3}-\frac{1}{256}\left(K_{1}+K_{2}\right) E \mathcal{E}^{2}+\frac{11}{768} K_{1} \mathcal{E} E^{2}-\frac{1}{64} K E^{3}$,

where

$$
\begin{aligned}
J & =\frac{1}{\Pi^{*}} A_{\mathrm{h} 1}, \\
J_{1} & =e \frac{1}{\Pi^{*}} \frac{\mathrm{d} A_{\mathrm{h} 1}}{\mathrm{~d} e}, \\
J_{2} & =e^{2} \frac{1}{\Pi^{*}} \frac{\mathrm{d}^{2} A_{\mathrm{h} 1}}{\mathrm{~d}^{2} e}, \\
K & =\frac{1}{\Pi^{*}} A_{\mathrm{h} 2}, \\
K_{1} & =e \frac{1}{\Pi^{*}} \frac{\mathrm{d} A_{\mathrm{h} 2}}{\mathrm{~d} e}, \\
K_{2} & =e^{2} \frac{1}{\Pi^{*}} \frac{\mathrm{d}^{2} A_{\mathrm{h} 2}}{\mathrm{~d}^{2} e},
\end{aligned}
$$

and

$$
\begin{aligned}
A_{\mathrm{h} 1} & =-\frac{1}{\cos i_{1}} A_{\mathrm{n} 1} \\
& =-\frac{2}{5} A_{\mathrm{G}}\left(1-e^{2}\right)^{1 / 2} I \frac{C}{C_{2}},
\end{aligned}
$$

while $A_{\mathrm{h} 2}$ could be derived from $A_{\mathrm{n} 2}$ in a similar manner. Here $J$ was treated as second order in $e$, while $J_{1,2}, K$, and $K_{1,2}$-s were considered as third order. To obtain the corresponding expressions for $u_{\mathrm{m} 1}, A_{\mathrm{h} 1,2}$ and its derivatives should be simply replaced by $-A_{\mathrm{n} 1,2}$ and derivatives. As these quantities will be used later, for the sake of the clarity we define them here:

$$
\begin{aligned}
L & =-\frac{1}{\Pi^{*}} A_{\mathrm{n} 1}, \\
L_{1} & =-e \frac{1}{\Pi^{*}} \frac{\mathrm{d} A_{\mathrm{n} 1}}{\mathrm{~d} e}, \\
L_{2} & =-e^{2} \frac{1}{\Pi^{*}} \frac{\mathrm{d}^{2} A_{\mathrm{n} 1}}{\mathrm{~d}^{2} e},
\end{aligned}
$$




$$
\begin{aligned}
M & =-\frac{1}{\Pi^{*}} A_{\mathrm{n} 2}, \\
M_{1} & =-e \frac{1}{\Pi^{*}} \frac{\mathrm{d} A_{\mathrm{n} 2}}{\mathrm{~d} e}, \\
M_{2} & =-e^{2} \frac{1}{\Pi^{*}} \frac{\mathrm{d}^{2} A_{\mathrm{n} 2}}{\mathrm{~d}^{2} e},
\end{aligned}
$$

Formally, similar expression can be written for the direct perturbative terms in the orbital motion $(\delta)$. Nevertheless, in this case the magnitude of the derivatives differ from those above, so we rewrite the results according to the orders of the direct terms as follows:

$\delta=\delta_{0}^{*}+D_{0} \mathcal{G}+D_{2} \sin 2 \mathcal{G}+D_{4} \sin 4 \mathcal{G}+D_{6} \sin 6 \mathcal{G}+D_{8} \sin 8 \mathcal{G}+\ldots$,

where

$$
\begin{aligned}
D_{0}= & V+\left(\frac{1}{16} V_{2}-\frac{1}{8} A_{1} V_{1}\right) \mathcal{E}^{2}+\frac{1}{4} V_{1} E \mathcal{E}-\frac{3}{1024} V_{2} \mathcal{E}^{4}-\frac{1}{256}\left(2 V_{1}-5 V_{2}\right) E \mathcal{E}^{3}+\frac{1}{256}\left(8 V_{1}+17 V_{2}\right) E^{2} \mathcal{E}^{2}+\frac{5}{32} V_{1} E^{3} \mathcal{E} \\
& +\frac{1}{4}\left(-W_{1}+A_{1} W_{0}\right) \mathcal{E}-\frac{1}{2} W E+\frac{1}{128}\left(W_{1}-W_{2}\right) \mathcal{E}^{3}-\frac{1}{32}\left(W_{1}+2 W_{2}\right) E \mathcal{E}^{2}-\frac{5}{32} W_{1} E^{2} \mathcal{E}-\frac{1}{8} W E^{3} \\
& +\left[V_{1}+\frac{1}{8}\left(V_{2}-2 A_{1} V_{1}-A_{2} V_{1}-2 A_{1} V_{2}\right) \mathcal{E}^{2}+\frac{1}{4}\left(V+1+V_{2}-2 A_{1} V_{1}\right) E \mathcal{E}\right. \\
& \left.-\frac{1}{4}\left(W_{1}+W_{2}-A_{1} W-A_{2} W-2 A_{1} W_{1}\right) \mathcal{E}-\frac{1}{2}\left(W_{1}-A_{1} W\right) E\right] \epsilon_{0} \\
& +\left[\frac{1}{2} V_{2}+\frac{1}{16} V_{2} \mathcal{E}^{2}+\frac{1}{4} V_{2} E \mathcal{E}-\frac{1}{4} W_{2} \mathcal{E}-\frac{1}{4} W_{2} E\right] \epsilon_{0}^{2}, \\
D_{2}= & -\frac{1}{4} V_{1} \mathcal{E}+\frac{1}{256}\left(2 V_{1}-2 V_{2}+5 A_{1} V_{1}+3 A_{2} V_{1}+6 A_{1} V_{2}\right) \mathcal{E}^{3}-\frac{1}{128}\left(6 V_{1}+6 V_{2}-7 A_{1} V_{1}\right) E \mathcal{E}^{2}-\frac{1}{16} V_{1} E^{2} \mathcal{E} \\
& +\frac{1}{2} W+\frac{1}{128}\left(2 W_{1}+6 W_{2}-A_{1} W-A_{2} W-10 A_{1} W_{1}\right) \mathcal{E}^{2}+\frac{1}{64}\left(10 W_{1}+7 A_{1} W\right) E \mathcal{E}-\frac{1}{8} W E^{2} \\
& +\left[-\frac{1}{4}\left(V_{1}+V_{2}+A_{1} V_{1}\right) \mathcal{E}+\frac{1}{128}\left(V_{1}-V_{2}\right) \mathcal{E}^{3}-\frac{3}{64}\left(V_{1}+3 V_{2}\right) E \mathcal{E}^{2}-\frac{1}{16}\left(V_{1}+V_{2}\right) E^{2} \mathcal{E}-\frac{1}{4} V_{1}\left(B_{1}+N_{1}\right)\right. \\
& \left.+\frac{1}{2} W_{1}+\frac{1}{64}\left(W_{1}+7 W_{2}\right) \mathcal{E}^{2}+\frac{5}{32}\left(W_{1}+W_{2}\right) E \mathcal{E}-\frac{1}{8} W_{1} E^{2}\right] \epsilon_{0} \\
& +\left[-\frac{1}{8}\left(2 V_{2}-2 A_{1} V_{1}-A_{2} V_{1}-2 A_{1} V_{2}\right) \mathcal{E}+\frac{1}{4} W_{2}\right] \epsilon_{0}^{2},
\end{aligned}
$$$$
D_{4}=\frac{1}{64}\left(V_{1}+V_{2}+A_{1} V_{1}\right) \mathcal{E}^{2}-\frac{1}{32} V_{1} E \mathcal{E}-\frac{1}{1536}\left(V_{1}+V_{2}\right) \mathcal{E}^{4}+\frac{1}{256}\left(V_{1}+2 V_{2}\right) E \mathcal{E}^{3}+\frac{1}{384}\left(V_{1}+V_{2}\right) E^{2} \mathcal{E}^{2}-\frac{1}{64} V_{1} E^{3} \mathcal{E}
$$$$
+\frac{1}{64} V_{1}\left(B_{1}+N_{1}\right) \mathcal{E}-\frac{1}{16}\left(W_{1}+A_{1} W\right) \mathcal{E}+\frac{1}{8} W E+\frac{1}{768}\left(W_{1}-3 W_{2}\right) \mathcal{E}^{3}-\frac{1}{128}\left(2 W_{1}+W_{2}\right) E \mathcal{E}^{2}+\frac{5}{192} W_{1} E^{2} \mathcal{E}
$$$$
+\left[\frac{1}{64}\left(V_{1}+3 V_{2}+A_{2} V_{1}-A_{1} V_{2}\right) \mathcal{E}^{2}-\frac{1}{32}\left(V_{1}+V_{2}-2 A_{1} V_{1}\right) E \mathcal{E}-\frac{1}{16}\left(W_{1}+W_{2}+A_{1} W+A_{2} W\right) \mathcal{E}+\frac{1}{8}\left(W_{1}-A_{1} W\right) E\right] \epsilon_{0}
$$$$
+\left[\frac{1}{32} V_{2} \mathcal{E}^{2}-\frac{1}{32} V_{2} E \mathcal{E}-\frac{1}{16} W_{2} \mathcal{E}+\frac{1}{16} W_{2} E\right] \epsilon_{0}^{2},
$$

$$
\begin{aligned}
D_{6}= & -\frac{1}{2304}\left(2 V_{1}+6 V_{2}+7 A_{1} V_{1}+1 A_{2} V_{1}+6 A_{1} V_{2}\right) \mathcal{E}^{3}+\frac{1}{1152}\left(6 V_{1}+6 V_{2}+7 A_{1} V_{1}\right) E \mathcal{E}^{2}-\frac{1}{144} V_{1} E^{2} \mathcal{E} \\
& +\frac{1}{384}\left(2 W_{1}+2 W_{2}+A_{1} W+A_{2} W+6 A_{1} W_{1}\right) \mathcal{E}^{2}-\frac{1}{192}\left(6 W_{1}+7 A_{1} W\right) E \mathcal{E}+\frac{1}{24} E^{2} W
\end{aligned}
$$$$
+\left[-\frac{1}{1152}\left(V_{1}+7 V_{2}\right) \mathcal{E}^{3}+\frac{1}{192}\left(V_{1}+3 V_{2}\right) E \mathcal{E}^{2}-\frac{1}{144}\left(V_{1}+V_{2}\right) E^{2} \mathcal{E}\right.
$$$$
\left.+\frac{1}{192}\left(W_{1}+3 W_{2}\right) \mathcal{E}^{2}-\frac{1}{32}\left(W_{1}+W_{2}\right) E \mathcal{E}+\frac{1}{24} W_{1} E^{2}\right] \epsilon_{0},
$$

$$
\begin{aligned}
D_{8}= & \frac{1}{24576}\left(V_{1}+7 V_{2}\right) \mathcal{E}^{4}-\frac{1}{2048}\left(V_{1}+3 V_{2}\right) E \mathcal{E}^{3}+\frac{11}{6144}\left(V_{1}+V_{2}\right) E^{2} \mathcal{E}^{2}-\frac{1}{512} V_{1} E^{3} \mathcal{E} \\
& -\frac{1}{3072}\left(W_{1}+3 W_{2}\right) \mathcal{E}^{3}+\frac{1}{256}\left(W_{1}+W_{2}\right) E \mathcal{E}^{2}-\frac{11}{768} W_{1} E^{2} \mathcal{E}+\frac{1}{64} W E^{3}
\end{aligned}
$$


In the above equations

$$
\begin{aligned}
V & =\frac{1}{\Pi^{*}} A_{\mathrm{d}}, \\
V_{1} & =e \frac{1}{\Pi^{*}} \frac{\mathrm{d} A_{\mathrm{d}}}{\mathrm{d} e}, \\
V_{2} & =e^{2} \frac{1}{\Pi^{*}} \frac{\mathrm{d}^{2} A_{\mathrm{d}}}{\mathrm{d} e^{2}}, \\
W & =\frac{1}{\Pi^{*}} B_{\mathrm{d}}, \\
W_{1} & =e \frac{1}{\Pi^{*}} \frac{\mathrm{d} B_{\mathrm{d}}}{\mathrm{d} e}, \\
W_{2} & =e^{2} \frac{1}{\Pi^{*}} \frac{\mathrm{d}^{2} B_{\mathrm{d}}}{\mathrm{d} e^{2}},
\end{aligned}
$$

where in our computations $V \mathrm{~s}$ were considered as first, while $W \mathrm{~s}$ as second order quantities in $E$ (or $\mathcal{E}, e$ ).

As a next step we calculate the angular elements in the observational system of references. We apply the following relations from the theory of spherical geometry:

$$
\begin{aligned}
\cos i & =\cos I_{0} \cos i_{1}-\sin I_{0} \sin i_{1} \cos h, \\
\mathrm{~d} u_{\mathrm{m}} & =\mathrm{d} h \frac{\cos i_{1}-\cos I_{0} \cos i}{1-\cos ^{2} i}-\mathrm{d} i_{1} \frac{\sin I_{0} \sin h \cos i}{1-\cos ^{2} i}, \\
\mathrm{~d} \Omega & =\mathrm{d} h \frac{\cos I_{0}-\cos i_{1} \cos i}{1-\cos ^{2} i}+\mathrm{d} i_{1} \frac{\sin I_{0} \sin h}{1-\cos ^{2} i}, \\
\mathrm{~d} i & =\mathrm{d} i_{1} \frac{\cos I_{0} \sin i_{1}+\cos i_{1} \sin I_{0} \cos h}{\sin i}-\mathrm{d} h \frac{\sin h \sin I_{0} \sin i_{1}}{\sin i} .
\end{aligned}
$$

By the use of these relations, after some algebra and the Taylorian expansion of the $1-\cos ^{2} i$ denominator we obtain

$$
\begin{aligned}
-\mathrm{d} \Omega \cos i= & \mathrm{d} h \cos i_{1}\left(C_{00}+C_{02} \cos 2 i_{1}+C_{04} \cos 4 i_{1}+C_{06} \cos 6 i_{1}\right) \\
& +\mathrm{d} h \cos h \sin i_{1}\left(C_{10}+C_{12} \cos 2 i_{1}+C_{14} \cos 4 i_{1}+C_{16} \cos 6 i_{1}\right) \\
& +\mathrm{d} h \cos 2 h \cos i_{1}\left(C_{20}+C_{22} \cos 2 i_{1}+C_{24} \cos 4 i_{1}+C_{26} \cos 6 i_{1}\right) \\
& +\mathrm{d} h \cos 3 h \sin i_{1}\left(C_{30}+C_{32} \cos 2 i_{1}+C_{34} \cos 4 i_{1}+C_{36} \cos 6 i_{1}\right) \\
& +\mathrm{d} h \cos 4 h \cos i_{1}\left(C_{40}+C_{42} \cos 2 i_{1}+C_{44} \cos 4 i_{1}+C_{46} \cos 6 i_{1}\right) \\
& +\mathrm{d} h \cos 5 h \sin i_{1}\left(C_{50}+C_{52} \cos 2 i_{1}+C_{54} \cos 4 i_{1}+C_{56} \cos 6 i_{1}\right) \\
& +\mathrm{d} h \cos 6 h \cos i_{1}\left(C_{60}+C_{62} \cos 2 i_{1}+C_{64} \cos 4 i_{1}+C_{66} \cos 6 i_{1}\right) \\
& -\mathrm{di} i_{1} \sin h \cos i_{1}\left(S_{10}+S_{12} \cos 2 i_{1}+S_{14} \cos 4 i_{1}\right)+\ldots,
\end{aligned}
$$

where

$$
\begin{aligned}
& C_{00}=\frac{765}{4096}-\frac{4725}{8192} \cos 2 I_{0}+\frac{357}{4096} \cos 4 I_{0}-\frac{91}{8192} \cos 6 I_{0}, \\
& C_{02}=\frac{1271}{8192}+\frac{8295}{16384} \cos 2 I_{0}-\frac{1743}{8192} \cos 4 I_{0}+\frac{329}{16384} \cos 6 I_{0}, \\
& C_{04}=\frac{117}{4096}+\frac{525}{8192} \cos 2 I_{0}+\frac{483}{4096} \cos 4 I_{0}-\frac{189}{8192} \cos 6 I_{0}, \\
& C_{06}=\frac{25}{8192}+\frac{105}{16384} \cos 2 I_{0}+\frac{63}{8192} \cos 4 I_{0}+\frac{231}{16384} \cos 6 I_{0}, \\
& C_{12}=-\frac{3449}{4096} \sin 2 I_{0}-\frac{31}{1024} \sin 4 I_{0}-\frac{5}{4096} \sin 6 I_{0}, \\
& C_{14}=-\frac{31}{512} \sin 2 I_{0}-\frac{27}{128} \sin 4 I_{0}-\frac{3}{512} \sin 6 I_{0}, \\
& C_{16}=-\frac{15}{4096} \sin 2 I_{0}-\frac{9}{1024} \sin 4 I_{0}-\frac{99}{4096} \sin 6 I_{0}, \\
& C_{20}=\frac{1271}{8192}-\frac{4549}{16384} \cos 2 I_{0}+\frac{1121}{8192} \cos 4 I_{0}-\frac{235}{16384} \cos 6 I_{0}, \\
& C_{22}=-\frac{1893}{16384}+\frac{10767}{32768} \cos 2 I_{0}-\frac{4003}{16384} \cos 4 I_{0}+\frac{1025}{32768} \cos 6 I_{0}, \\
& C_{24}=-\frac{287}{8192}-\frac{707}{16384} \cos 2 I_{0}+\frac{903}{8192} \cos 4 I_{0}-\frac{525}{16384} \cos 6 I_{0},
\end{aligned}
$$


$C_{26}=-\frac{75}{16384}-\frac{255}{32768} \cos 2 I_{0}-\frac{45}{16384} \cos 4 I_{0}+\frac{495}{32768} \cos 6 I_{0}$,

$C_{32}=-\frac{501}{8192} \sin 2 I_{0}+\frac{57}{2048} \sin 4 I_{0}+\frac{15}{8192} \sin 6 I_{0}$,

$C_{34}=\frac{57}{1024} \sin 2 I_{0}-\frac{9}{256} \sin 4 I_{0}+\frac{5}{1024} \sin 6 I_{0}$,

$C_{36}=\frac{45}{8192} \sin 2 I_{0}+\frac{15}{2048} \sin 4 I_{0}-\frac{55}{8192} \sin 6 I_{0}$,

$C_{40}=\frac{117}{4096}-\frac{387}{8192} \cos 2 I_{0}+\frac{99}{4096} \cos 4 I_{0}-\frac{45}{8192} \cos 6 I_{0}$,

$C_{42}=-\frac{287}{8192}+\frac{1057}{16384} \cos 2 I_{0}-\frac{329}{8192} \cos 4 I_{0}+\frac{175}{16384} \cos 6 I_{0}$,

$C_{44}=\frac{19}{4096}-\frac{149}{8192} \cos 2 I_{0}+\frac{85}{4096} \cos 4 I_{0}-\frac{59}{8192} \cos 6 I_{0}$,

$C_{46}=\frac{15}{8192}+\frac{15}{16384} \cos 2 I_{0}-\frac{39}{8192} \cos 4 I_{0}+\frac{33}{16384} \cos 6 I_{0}$,

$C_{52}=-\frac{25}{8192} \sin 2 I_{0}+\frac{5}{2048} \sin 4 I_{0}-\frac{5}{8192} \sin 6 I_{0}$,

$C_{54}=\frac{5}{1024} \sin 2 I_{0}-\frac{1}{256} \sin 4 I_{0}+\frac{1}{1024} \sin 6 I_{0}$,

$C_{56}=-\frac{15}{8192} \sin 2 I_{0}+\frac{3}{2048} \sin 4 I_{0}-\frac{3}{8192} \sin 6 I_{0}$,

$C_{60}=\frac{25}{8192}-\frac{75}{16384} \cos 2 I_{0}+\frac{15}{8192} \cos 4 I_{0}-\frac{5}{16384} \cos 6 I_{0}$,

$C_{62}=-\frac{75}{16382}+\frac{225}{32768} \cos 2 I_{0}-\frac{45}{16384} \cos 4 I_{0}+\frac{15}{32768} \cos 6 I_{0}$,

$C_{64}=\frac{15}{8192}-\frac{45}{16384} \cos 2 I_{0}+\frac{9}{8192} \cos 4 I_{0}-\frac{3}{16384} \cos 6 I_{0}$,

$C_{66}=-\frac{5}{16384}+\frac{15}{32768} \cos 2 I_{0}-\frac{3}{16384} \cos 4 I_{0}+\frac{1}{32768} \cos 6 I_{0}$,

$S_{10}=-\frac{1727}{2048} \sin 2 I_{0}-\frac{17}{512} \sin 4 I_{0}-\frac{19}{2048} \sin 6 I_{0}$,

$S_{12}=-\frac{31}{512} \sin 2 I_{0}-\frac{27}{128} \sin 4 I_{0}-\frac{3}{512} \sin 6 I_{0}$,

$S_{14}=-\frac{5}{2048} \sin 2 I_{0}-\frac{3}{512} \sin 4 I_{0}-\frac{33}{2048} \sin 6 I_{0}$

As Eq. 29) reveals there is only a very small cyclic variation in $i_{1}$. Consequently, in Eq. A.86) $i_{1}$ can be considered as constant. With this approximation the integration is a very easy task, and we do not feel the necessity to report its result here. (Nevertheless, it can be read directly - with the exclusion of the secular term - from the $V_{0 n m}$ coefficients [Eqs. (A.211) - A.225] of the final form of the O-C given below [Eq. A.123] ].) Instead we list the analytical form of the argument of periastron in the observational frame of reference $(\omega)$ :

$$
\begin{aligned}
\omega= & g+u_{\mathrm{m}}, \\
= & \omega_{0}^{*}+O_{0} \mathcal{G}+O_{2} \sin 2 \mathcal{G}+O_{4} \sin 4 \mathcal{G}+O_{6} \sin 6 \mathcal{G}+O_{8} \sin 8 \mathcal{G} \\
& +O_{n 0} \sin \left(n h_{0}^{*}+n H_{0} \mathcal{G}\right)+O_{n \mp m} \sin \left[n h_{0}^{*}+\left(n H_{0} \mp m\right) \mathcal{G}\right],
\end{aligned}
$$

where

$$
\begin{aligned}
O_{0}= & 1+U_{0}\left(1+C_{0}\right), \\
O_{n}= & G_{n}+U_{n}\left(1+C_{0}\right), \\
O_{n 0}= & \frac{1}{n} C_{n}, \\
O_{n \mp 2}= & \pm C_{n}\left\{\frac{1}{4} K+\frac{1}{8}\left(J_{1}+K_{1}\right) \mathcal{E}+\frac{1}{128}\left(K_{1}+3 K_{2}\right) \mathcal{E}^{2}+\frac{5}{64} K_{1} E \mathcal{E}-\frac{1}{16} K E^{2}\right. \\
& \left.+\left[\frac{1}{4} K_{1}+\frac{1}{8}\left(J_{1}+J_{2}+K_{1}+K_{2}\right) \mathcal{E}\right] \epsilon_{0}+\frac{1}{8} K_{2} \epsilon_{0}^{2}\right\}, \\
O_{n \mp 4}= & \pm C_{n}\left\{-\frac{1}{32}\left(K_{1}+A_{1} K\right) \mathcal{E}+\frac{1}{16} K E-\frac{1}{128}\left(J_{1}+J_{2}+K_{1}+K_{2}\right) \mathcal{E}^{2}+\frac{1}{64}\left(J_{1}+K_{1}\right) E \mathcal{E}\right.
\end{aligned}
$$




$$
\begin{gathered}
\left.+\left[-\frac{1}{32}\left(K_{1}+K_{2}\right) \mathcal{E}+\frac{1}{16} K_{1} E\right] \epsilon_{0}\right\}, \\
O_{n \mp 6}= \pm C_{n}\left[\frac{1}{384}\left(K_{1}+K_{2}\right) \mathcal{E}^{2}-\frac{1}{64} K_{1} E \mathcal{E}+\frac{1}{48} K E^{2}\right],
\end{gathered}
$$

and, furthermore, we applied the following abbreviations:

$C_{0}=C_{00}+C_{02} \cos 2 i_{1}+C_{04} \cos 4 i_{1}+C_{06} \cos 6 i_{1}$,

but e.g.

$C_{1}=\sin i_{1}\left(C_{10}+C_{12} \cos 2 i_{1}+C_{14} \cos 4 i_{1}+C_{16} \cos 6 i_{1}\right)$,

i.e. at the trigonometric terms the $\sin i_{1}$ or $\cos i_{1}$ multiplicators outside the parenthesis are also included. (Note, in the following for the sake of the simplicity we consider $C_{n} \mathrm{~s}$ as being in the order of $e^{n}$. This is not necessarily exactly correct, but as $C_{n} \mathrm{~s}$ come from the Taylorian expansion of $1-\cos ^{2} i$ it may be partly verified.)

We are now in the position to give the final analytic form of the $\mathrm{O}-\mathrm{C}$ curve up to the fifth order. This is given as follows:

$$
\begin{aligned}
& \frac{2 \pi}{P} O-C=V_{100} \cos \left(\omega_{0}^{*}+O_{0} \mathcal{G}\right)+V_{200} \sin \left(2 \omega_{0}^{*}+2 O_{0} \mathcal{G}\right)+V_{300} \cos \left(3 \omega^{*}+3 O_{0} \mathcal{G}\right) \\
& +V_{400} \sin \left(4 \omega_{0}^{*}+4 O_{0} \mathcal{G}\right)+V_{500} \cos \left(5 \omega^{*}+5 O_{0} \mathcal{G}\right) \\
& +V_{120} \cos \left[\omega_{0}^{*}+\left(O_{0}+2\right) \mathcal{G}\right]+V_{1-20} \cos \left[\omega_{0}^{*}+\left(O_{0}-2\right) \mathcal{G}\right] \\
& +V_{140} \cos \left[\omega_{0}^{*}+\left(O_{0}+4\right) \mathcal{G}\right]+V_{1-40} \cos \left[\omega_{0}^{*}+\left(O_{0}-4\right) \mathcal{G}\right] \\
& +V_{160} \cos \left[\omega_{0}^{*}+\left(O_{0}+6\right) \mathcal{G}\right]+V_{1-60} \cos \left[\omega_{0}^{*}+\left(O_{0}-6\right) \mathcal{G}\right] \\
& +V_{180} \cos \left[\omega_{0}^{*}+\left(O_{0}+8\right) \mathcal{G}\right]+V_{1-80} \cos \left[\omega_{0}^{*}+\left(O_{0}-8\right) \mathcal{G}\right] \\
& +V_{220} \sin \left[2 \omega_{0}^{*}+\left(2 O_{0}+2\right) \mathcal{G}\right]+V_{2-20} \sin \left[2 \omega_{0}^{*}+\left(2 O_{0}-2\right) \mathcal{G}\right] \\
& +V_{240} \sin \left[2 \omega_{0}^{*}+\left(2 O_{0}+4\right) \mathcal{G}\right]+V_{2-40} \sin \left[2 \omega_{0}^{*}+\left(2 O_{0}-4\right) \mathcal{G}\right] \\
& +V_{260} \sin \left[2 \omega_{0}^{*}+\left(2 O_{0}+6\right) \mathcal{G}\right]+V_{2-60} \sin \left[2 \omega_{0}^{*}+\left(2 O_{0}-6\right) \mathcal{G}\right] \\
& +V_{320} \sin \left[3 \omega_{0}^{*}+\left(3 O_{0}+2\right) \mathcal{G}\right]+V_{2-20} \sin \left[3 \omega_{0}^{*}+\left(3 O_{0}-2\right) \mathcal{G}\right] \\
& +V_{340} \sin \left[3 \omega_{0}^{*}+\left(3 O_{0}+4\right) \mathcal{G}\right]+V_{2-40} \sin \left[3 \omega_{0}^{*}+\left(3 O_{0}-4\right) \mathcal{G}\right] \\
& +V_{420} \sin \left[4 \omega_{0}^{*}+\left(4 O_{0}+2\right) \mathcal{G}\right]+V_{4-20} \sin \left[4 \omega_{0}^{*}+\left(4 O_{0}-2\right) \mathcal{G}\right] \\
& +V_{101} \cos \left[\omega_{0}^{*}+h_{0}^{*}+\left(O_{0}+H_{0}\right) \mathcal{G}\right]+V_{10-1} \cos \left[\omega_{0}^{*}-h_{0}^{*}+\left(O_{0}-H_{0}\right) \mathcal{G}\right] \\
& +V_{102} \cos \left[\omega_{0}^{*}+2 h_{0}^{*}+\left(O_{0}+2 H_{0}\right) \mathcal{G}\right]+V_{10-2} \cos \left[\omega_{0}^{*}-2 h_{0}^{*}+\left(O_{0}-2 H_{0}\right) \mathcal{G}\right] \\
& +V_{103} \cos \left[\omega_{0}^{*}+3 h_{0}^{*}+\left(O_{0}+3 H_{0}\right) \mathcal{G}\right]+V_{10-3} \cos \left[\omega_{0}^{*}-3 h_{0}^{*}+\left(O_{0}-3 H_{0}\right) \mathcal{G}\right] \\
& +V_{104} \cos \left[\omega_{0}^{*}+4 h_{0}^{*}+\left(O_{0}+4 H_{0}\right) \mathcal{G}\right]+V_{10-4} \cos \left[\omega_{0}^{*}-4 h_{0}^{*}+\left(O_{0}-4 H_{0}\right) \mathcal{G}\right] \\
& +V_{121} \cos \left[\omega_{0}^{*}+h_{0}^{*}+\left(O_{0}+2+H_{0}\right) \mathcal{G}\right]+V_{12-1} \cos \left[\omega_{0}^{*}-h_{0}^{*}+\left(O_{0}+2-H_{0}\right) \mathcal{G}\right] \\
& +V_{1-21} \cos \left[\omega_{0}^{*}+h_{0}^{*}+\left(O_{0}-2+H_{0}\right) \mathcal{G}\right]+V_{1-2-1} \cos \left[\omega_{0}^{*}-h_{0}^{*}+\left(O_{0}-2-H_{0}\right) \mathcal{G}\right] \\
& +V_{122} \cos \left[\omega_{0}^{*}+2 h_{0}^{*}+\left(O_{0}+2+2 H_{0}\right) \mathcal{G}\right]+V_{12-2} \cos \left[\omega_{0}^{*}-2 h_{0}^{*}+\left(O_{0}+2-2 H_{0}\right) \mathcal{G}\right] \\
& +V_{1-22} \cos \left[\omega_{0}^{*}+2 h_{0}^{*}+\left(O_{0}-2+2 H_{0}\right) \mathcal{G}\right]+V_{1-2-2} \cos \left[\omega_{0}^{*}-2 h_{0}^{*}+\left(O_{0}-2-2 H_{0}\right) \mathcal{G}\right] \\
& +V_{123} \cos \left[\omega_{0}^{*}+3 h_{0}^{*}+\left(O_{0}+2+3 H_{0}\right) \mathcal{G}\right]+V_{12-3} \cos \left[\omega_{0}^{*}-3 h_{0}^{*}+\left(O_{0}+2-3 H_{0}\right) \mathcal{G}\right] \\
& +V_{1-23} \cos \left[\omega_{0}^{*}+3 h_{0}^{*}+\left(O_{0}-2+3 H_{0}\right) \mathcal{G}\right]+V_{1-2-3} \cos \left[\omega_{0}^{*}-3 h_{0}^{*}+\left(O_{0}-2-3 H_{0}\right) \mathcal{G}\right] \\
& +V_{141} \cos \left[\omega_{0}^{*}+h_{0}^{*}+\left(O_{0}+4+H_{0}\right) \mathcal{G}\right]+V_{14-1} \cos \left[\omega_{0}^{*}-h_{0}^{*}+\left(O_{0}+4-H_{0}\right) \mathcal{G}\right] \\
& +V_{1-41} \cos \left[\omega_{0}^{*}+h_{0}^{*}+\left(O_{0}-4+H_{0}\right) \mathcal{G}\right]+V_{1-2-1} \cos \left[\omega_{0}^{*}-h_{0}^{*}+\left(O_{0}-4-H_{0}\right) \mathcal{G}\right] \\
& +V_{142} \cos \left[\omega_{0}^{*}+2 h_{0}^{*}+\left(O_{0}+4+2 H_{0}\right) \mathcal{G}\right]+V_{12-2} \cos \left[\omega_{0}^{*}-2 h_{0}^{*}+\left(O_{0}+4-2 H_{0}\right) \mathcal{G}\right] \\
& +V_{1-42} \cos \left[\omega_{0}^{*}+2 h_{0}^{*}+\left(O_{0}-4+2 H_{0}\right) \mathcal{G}\right]+V_{1-2-2} \cos \left[\omega_{0}^{*}-2 h_{0}^{*}+\left(O_{0}-4-2 H_{0}\right) \mathcal{G}\right] \\
& +V_{161} \cos \left[\omega_{0}^{*}+h_{0}^{*}+\left(O_{0}+6+H_{0}\right) \mathcal{G}\right]+V_{16-1} \cos \left[\omega_{0}^{*}-h_{0}^{*}+\left(O_{0}+6-H_{0}\right) \mathcal{G}\right] \\
& +V_{1-61} \cos \left[\omega_{0}^{*}+h_{0}^{*}+\left(O_{0}-6+H_{0}\right) \mathcal{G}\right]+V_{1-6-1} \cos \left[\omega_{0}^{*}-h_{0}^{*}+\left(O_{0}-6-H_{0}\right) \mathcal{G}\right] \\
& +V_{201} \sin \left[2 \omega_{0}^{*}+h_{0}^{*}+\left(2 O_{0}+H_{0}\right) \mathcal{G}\right]+V_{20-1} \sin \left[2 \omega_{0}^{*}-h_{0}^{*}+\left(2 O_{0}-H_{0}\right) \mathcal{G}\right] \\
& +V_{202} \sin \left[2 \omega_{0}^{*}+2 h_{0}^{*}+\left(2 O_{0}+2 H_{0}\right) \mathcal{G}\right]+V_{20-1} \sin \left[2 \omega_{0}^{*}-2 h_{0}^{*}+\left(2 O_{0}-2 H_{0}\right) \mathcal{G}\right] \\
& +V_{203} \sin \left[2 \omega_{0}^{*}+3 h_{0}^{*}+\left(2 O_{0}+3 H_{0}\right) \mathcal{G}\right]+V_{20-1} \sin \left[2 \omega_{0}^{*}-3 h_{0}^{*}+\left(2 O_{0}-3 H_{0}\right) \mathcal{G}\right] \\
& +V_{221} \sin \left[2 \omega_{0}^{*}+h_{0}^{*}+\left(2 O_{0}+2+H_{0}\right) \mathcal{G}\right]+V_{22-1} \sin \left[2 \omega_{0}^{*}-h_{0}^{*}+\left(2 O_{0}+2-H_{0}\right) \mathcal{G}\right] \\
& +V_{2-21} \sin \left[2 \omega_{0}^{*}+h_{0}^{*}+\left(2 O_{0}-2+H_{0}\right) \mathcal{G}\right]+V_{2-2-1} \sin \left[2 \omega_{0}^{*}-h_{0}^{*}+\left(2 O_{0}-2-H_{0}\right) \mathcal{G}\right] \\
& +V_{222} \sin \left[2 \omega_{0}^{*}+2 h_{0}^{*}+\left(2 O_{0}+2+2 H_{0}\right) \mathcal{G}\right]+V_{22-2} \sin \left[2 \omega_{0}^{*}-2 h_{0}^{*}+\left(2 O_{0}+2-2 H_{0}\right) \mathcal{G}\right] \\
& +V_{2-22} \sin \left[2 \omega_{0}^{*}+2 h_{0}^{*}+\left(2 O_{0}-2+2 H_{0}\right) \mathcal{G}\right]+V_{2-2-2} \sin \left[2 \omega_{0}^{*}-2 h_{0}^{*}+\left(2 O_{0}-2-2 H_{0}\right) \mathcal{G}\right] \\
& +V_{241} \sin \left[2 \omega_{0}^{*}+h_{0}^{*}+\left(2 O_{0}+4+H_{0}\right) \mathcal{G}\right]+V_{24-1} \sin \left[2 \omega_{0}^{*}-h_{0}^{*}+\left(2 O_{0}+4-H_{0}\right) \mathcal{G}\right] \\
& +V_{2-41} \sin \left[2 \omega_{0}^{*}+h_{0}^{*}+\left(2 O_{0}-4+H_{0}\right) \mathcal{G}\right]+V_{2-4-1} \sin \left[2 \omega_{0}^{*}-h_{0}^{*}+\left(2 O_{0}-4-H_{0}\right) \mathcal{G}\right]
\end{aligned}
$$


$+V_{301} \cos \left[3 \omega_{0}^{*}+h_{0}^{*}+\left(3 O_{0}+H_{0}\right) \mathcal{G}\right]+V_{30-1} \cos \left[3 \omega_{0}^{*}-h_{0}^{*}+\left(3 O_{0}-H_{0}\right) \mathcal{G}\right]$

$+V_{302} \cos \left[3 \omega_{0}^{*}+2 h_{0}^{*}+\left(3 O_{0}+2 H_{0}\right) \mathcal{G}\right]+V_{30-2} \cos \left[3 \omega_{0}^{*}-2 h_{0}^{*}+\left(3 O_{0}-2 H_{0}\right) \mathcal{G}\right]$

$+V_{321} \cos \left[3 \omega_{0}^{*}+h_{0}^{*}+\left(3 O_{0}+2+H_{0}\right) \mathcal{G}\right]+V_{32-1} \cos \left[3 \omega_{0}^{*}-h_{0}^{*}+\left(3 O_{0}+2-H_{0}\right) \mathcal{G}\right]$

$+V_{3-21} \cos \left[3 \omega_{0}^{*}+h_{0}^{*}+\left(3 O_{0}-2+H_{0}\right) \mathcal{G}\right]+V_{3-2-1} \cos \left[3 \omega_{0}^{*}-h_{0}^{*}+\left(3 O_{0}-2-H_{0}\right) \mathcal{G}\right]$

$+V_{401} \sin \left[4 \omega_{0}^{*}+h_{0}^{*}+\left(4 O_{0}+H_{0}\right) \mathcal{G}\right]+V_{40-1} \sin \left[4 \omega_{0}^{*}-h_{0}^{*}+\left(4 O_{0}-H_{0}\right) \mathcal{G}\right]$

$+V_{020} \sin 2 \mathcal{G}+V_{040} \sin 4 \mathcal{G}+V_{060} \sin 6 \mathcal{G}+V_{080} \sin 8 \mathcal{G}$

$+V_{001} \sin \left(h_{0}^{*}+H_{0} \mathcal{G}\right)+V_{002} \sin \left(2 h_{0}^{*}+2 H_{0} \mathcal{G}\right)+V_{003} \sin \left(3 h_{0}^{*}+3 H_{0} \mathcal{G}\right)$

$+V_{004} \sin \left(4 h_{0}^{*}+4 H_{0} \mathcal{G}\right)+V_{005} \sin \left(5 h_{0}^{*}+5 H_{0} \mathcal{G}\right)$

$+V_{021} \sin \left[h_{0}^{*}+\left(H_{0}+2\right) \mathcal{G}\right]+V_{0-21} \sin \left[h_{0}^{*}+\left(H_{0}-2\right) \mathcal{G}\right]$

$+V_{022} \sin \left[2 h_{0}^{*}+\left(2 H_{0}+2\right) \mathcal{G}\right]+V_{0-22} \sin \left[2 h_{0}^{*}+\left(2 H_{0}-2\right) \mathcal{G}\right]+$

$+V_{041} \sin \left[h_{0}^{*}+\left(H_{0}+4\right) \mathcal{G}\right]+V_{0-41} \sin \left[h_{0}^{*}+\left(H_{0}-4\right) \mathcal{G}\right]+O\left(e^{6}, E^{6}, \mathcal{E}^{6}\right)$,

where

$$
\begin{array}{rl}
V_{100}= & j e_{0}\left\{-2+\frac{1}{32}\left(8 A_{1}+A_{1}^{2}\right) \mathcal{E}^{2}-\frac{1}{8}\left(4+A_{1}\right) E \mathcal{E}+\frac{1}{8} E^{2}+\frac{1}{64} E \mathcal{E}^{3}-\frac{17}{256} E^{2} \mathcal{E}^{2}-\frac{37}{128} E^{3} \mathcal{E}+\frac{35}{512} E^{4}\right. \\
& +\left(\frac{1}{2}+\frac{1}{8} E \mathcal{E}-\frac{1}{32} E^{2}\right) \mathcal{C}_{1}^{2}-\frac{1}{32} C_{1}^{4}+\frac{1}{8} C_{2}^{2}-\frac{1}{4} M E\left(1+C_{0}\right) \\
& \left.+\left[-2+\frac{1}{4}\left(2 A_{1}+A_{2}\right) E^{2}-\frac{1}{8}\left(4-6 A_{1}+A_{2}\right) E \mathcal{E}+\frac{1}{8}\left(1-2 A_{1}\right) E^{2}+\frac{1}{2} C_{1}^{2}\right] \epsilon_{0}\right\}, \\
V_{1-20}= & j e_{0}\left\{\frac{1}{4}\left(2-A_{1}\right) \mathcal{E}+\frac{1}{2} E-\frac{1}{256}\left(4+7 A_{1}+9 A_{2}\right) \mathcal{E}^{3}+\frac{1}{256}\left(20-71 A_{1}-21 A_{2}\right) E \mathcal{E}^{2}+\frac{1}{64}\left(15-22 A_{1}\right) E^{2} \mathcal{E}+\frac{9}{64} E^{3}\right. \\
& -\left[\frac{1}{16}\left(2-A_{1}\right) \mathcal{E}+\frac{1}{8} E\right] C_{1}^{2}-\left[\frac{1}{2} M+\frac{1}{4}\left(L_{1}+M_{1}\right) \mathcal{E}\right]\left(1+C_{0}\right) \\
& +\left[\frac{1}{4}\left(2-4 A_{1}-A_{2}+2 A_{1}^{2}\right) \mathcal{E}+\frac{1}{2}\left(1-A_{1}\right) E-\frac{1}{64} \mathcal{E}^{3}+\frac{5}{64} E \mathcal{E}^{2}+\frac{15}{64} E^{2} \mathcal{E}+\frac{9}{64} E^{3}+B_{1}+\frac{1}{2} N_{1}\right. \\
& \left.\left.-\left(\frac{1}{8} \mathcal{E}+\frac{1}{8} E\right) C_{1}^{2}-\frac{1}{2}\left(M+M_{1}\right)\left(1+C_{0}\right)\right] \epsilon_{0}+\left[-\frac{3}{4}\left(A_{1}+A_{2}\right) \mathcal{E}-\frac{1}{4}\left(2 A_{1}+A_{2}\right) E\right] \epsilon_{0}^{2}\right\}, \\
V_{120} & j e_{0}\left\{\frac{1}{4}\left(2+A_{1}\right) \mathcal{E}-\frac{1}{2} E-\frac{1}{256}\left(4+13 A_{1}+3 A_{2}\right) \mathcal{E}^{3}+\frac{1}{256}\left(28+25 A_{1}+23 A_{2}\right) E \mathcal{E}^{2}-\frac{1}{64}\left(1-17 A_{1}\right) E^{2} \mathcal{E}-\frac{5}{64} E^{3}\right. \\
& +\left[-\frac{1}{16}\left(2+A_{1}\right) \mathcal{E}+\frac{1}{8} E\right] C_{1}^{2}+\left[\frac{1}{2} M+\frac{1}{4}\left(L_{1}+M_{1}\right) \mathcal{E}\right]\left(1+C_{0}\right) \\
& +\left[\frac{1}{4}\left(2+A_{2}-2 A_{1}^{2}\right) \mathcal{E}-\frac{1}{2}\left(1-A_{1}\right) E-\frac{1}{64} \mathcal{E}^{3}+\frac{7}{64} E \mathcal{E}^{2}-\frac{1}{64} E^{2} \mathcal{E}-\frac{5}{64} E^{3}+\frac{1}{2} N_{1}\right. \\
& \left.\left.+\left(-\frac{1}{8} \mathcal{E}+\frac{1}{8} E\right) C_{1}^{2}+\frac{1}{2}\left(M+M_{1}\right)\left(1+C_{0}\right)\right] \epsilon_{0}+\left[\frac{1}{4}\left(-A_{1}+A_{2}\right) \mathcal{E}+\frac{1}{4}\left(2 A_{1}+A_{2}\right) E\right] \epsilon_{0}^{2}\right\},
\end{array}
$$

$$
\begin{aligned}
V_{1-40}= & j e_{0}\left\{\frac{1}{64}\left(-4+A_{1}+A_{2}\right) \mathcal{E}^{2}-\frac{1}{32} A_{1} \mathcal{E} E+\frac{1}{16} E^{2}+\frac{1}{384} \mathcal{E}^{4}-\frac{1}{96} E \mathcal{E}^{3}-\frac{29}{768} E^{2} \mathcal{E}^{2}+\frac{1}{96} E^{3} \mathcal{E}+\frac{9}{256} E^{4}\right. \\
& -\left(\frac{1}{8} B_{1}+\frac{1}{16} N_{1}\right) \mathcal{E}+\left(\frac{1}{64} \mathcal{E}^{2}-\frac{1}{64} E^{2}\right) \mathcal{C}_{1}^{2}+\left(\frac{1}{8} M+\frac{1}{16} M_{1}\right) \mathcal{E}\left(1+C_{0}\right) \\
& \left.+\left[\frac{1}{32}\left(-2+5 A_{1}+2 A_{2}\right) \mathcal{E}^{2}-\frac{1}{32}\left(2 A_{1}+A_{2}\right) E \mathcal{E}+\frac{1}{16}\left(1-2 A_{1}\right) E^{2}\right] \epsilon_{0}\right\}, \\
V_{140}= & j e_{0}\left\{-\frac{1}{64}\left(4+9 A_{1}+A_{2}+2 A_{1}^{2}\right) \mathcal{E}^{2}+\frac{1}{32}\left(8+5 A_{1}\right) E \mathcal{E}-\frac{3}{16} E^{2}+\frac{1}{384} \mathcal{E}^{4}-\frac{1}{48} E \mathcal{E}^{3}+\frac{19}{768} E^{2} \mathcal{E}^{2}+\frac{13}{192} E^{3} \mathcal{E}-\frac{19}{256} E^{4}\right. \\
& -\frac{1}{16} \mathcal{E} N_{1}+\left(\frac{1}{64} \mathcal{E}^{2}-\frac{1}{16} E \mathcal{E}+\frac{3}{64} E^{2}\right) C_{1}^{2}+\left[-\left(\frac{1}{8} M+\frac{1}{16} M_{1}\right) \mathcal{E}+\frac{1}{4} M E\right]\left(1+C_{0}\right) \\
& \left.+\left[-\frac{1}{32}\left(2+5 A_{1}+6 A_{2}\right) \mathcal{E}^{2}+\frac{1}{32}\left(8-6 A_{1}+5 A_{2}\right) E \mathcal{E}+\frac{3}{16}\left(-1+2 A_{1}\right) E^{2}\right] \epsilon_{0}\right\},
\end{aligned}
$$

$V_{1-60}=j e_{0}\left\{\frac{1}{2304}\left(12+13 A_{1}-9 A_{2}\right) \mathcal{E}^{3}+\frac{1}{768}\left(-12+5 A_{1}+3 A_{2}\right) \mathcal{E}^{2} E-\frac{1}{576}\left(3+7 A_{1}\right) \mathcal{E} E^{2}+\frac{1}{64} E^{3}\right.$ 


$$
\begin{aligned}
& \left.+\left[\frac{1}{192} \mathcal{E}^{3}-\frac{1}{64} \mathcal{E}^{2} E-\frac{1}{192} \mathcal{E} E^{2}+\frac{1}{64} E^{3}\right] \epsilon_{0}\right\}, \\
& V_{160}=j e_{0}\left\{\frac{1}{2304}\left(12+71 A_{1}+21 A_{2}\right) \mathcal{E}^{3}-\frac{1}{768}\left(36+91 A_{1}+9 A_{2}\right) \mathcal{E}^{2} E+\frac{1}{576}\left(69+52 A_{1}\right) \mathcal{E} E^{2}-\frac{5}{64} E^{3}\right. \\
& \left.+\left[\frac{1}{192} \mathcal{E}^{3}-\frac{3}{64} \mathcal{E}^{2} E+\frac{23}{192} \mathcal{E} E^{2}-\frac{5}{64} E^{3}\right] \epsilon_{0}\right\}, \\
& V_{1-80}=j e_{0}\left\{-\frac{1}{3072} \mathcal{E}^{4}+\frac{1}{384} \mathcal{E}^{3} E-\frac{7}{1536} E^{2} \mathcal{E}^{2}-\frac{1}{384} \mathcal{E} E^{3}+\frac{5}{1024} E^{4}\right\}, \\
& V_{180}=j e_{0}\left\{-\frac{1}{3072} \mathcal{E}^{4}+\frac{1}{192} \mathcal{E}^{3} E-\frac{43}{1536} E^{2} \mathcal{E}^{2}+\frac{11}{192} \mathcal{E} E^{3}-\frac{35}{1024} E^{4}\right\}, \\
& V_{10-1}=j e_{0} C_{1}\left\{1-\frac{1}{8} A_{1} \mathcal{E}^{2}+\frac{1}{16}\left(4+A_{1}\right) \mathcal{E} E-\frac{1}{16} E^{2}-\frac{1}{8} C_{1}^{2}+\frac{1}{4} C_{2}\right. \\
& \left.+\left[1+\frac{1}{4} \varepsilon E-\frac{1}{16} E^{2}-\frac{1}{8} C_{1}^{2}+\frac{1}{4} C_{2}\right] \epsilon_{0}\right\}, \\
& V_{101}=j e_{0} C_{1}\left\{-1+\frac{1}{8} A_{1} \mathcal{E}^{2}-\frac{1}{16}\left(4+A_{1}\right) \mathcal{E} E+\frac{1}{16} E^{2}+\frac{1}{8} C_{1}^{2}+\frac{1}{4} C_{2}\right. \\
& \left.+\left[-1-\frac{1}{4} \mathcal{E} E+\frac{1}{16} E^{2}+\frac{1}{8} C_{1}^{2}+\frac{1}{4} C_{2}\right] \epsilon_{0}\right\}, \\
& V_{1-2-1}=j e_{0} C_{1}\left\{\frac{1}{8}\left(-2+A_{1}\right) \mathcal{E}-\frac{1}{4} E+\frac{1}{128} \mathcal{E}^{3}-\frac{5}{128} \mathcal{E}^{2} E-\frac{15}{128} \mathcal{E} E^{2}-\frac{9}{128} E^{3}\right. \\
& +\left(\frac{1}{32} \mathcal{E}+\frac{1}{32} E\right) C_{1}^{2}-\left(\frac{1}{16} \mathcal{E}+\frac{1}{16} E\right) C_{2}-\frac{1}{4} K+\frac{1}{4} M\left(1+C_{0}\right) \\
& \left.+\left[\frac{1}{8}\left(-2+4 A_{1}+A_{2}\right) \mathcal{E}+\frac{1}{4}\left(-1+A_{1}\right) E\right] \epsilon_{0}\right\}, \\
& V_{1-21}=j e_{0} C_{1}\left\{\frac{1}{8}\left(2-A_{1}\right) \mathcal{E}+\frac{1}{4} E-\frac{1}{128} \mathcal{E}^{3}+\frac{5}{128} \mathcal{E}^{2} E+\frac{15}{128} \mathcal{E} E^{2}+\frac{9}{128} E^{3}\right. \\
& -\left(\frac{1}{32} \mathcal{E}+\frac{1}{32} E\right) C_{1}^{2}-\left(\frac{1}{16} \mathcal{E}+\frac{1}{16} E\right) C_{2}-\frac{1}{4} K-\frac{1}{4} M\left(1+C_{0}\right) \\
& \left.+\left[\frac{1}{8}\left(2-4 A_{1}-A_{2}\right) \mathcal{E}+\frac{1}{4}\left(1-A_{1}\right) E\right] \epsilon_{0}\right\}, \\
& V_{12-1}=j e_{0} C_{1}\left\{-\frac{1}{8}\left(2+A_{1}\right) \mathcal{E}+\frac{1}{4} E+\frac{1}{128} \mathcal{E}^{3}-\frac{7}{128} \mathcal{E}^{2} E+\frac{1}{128} \mathcal{E} E^{2}+\frac{5}{128} E^{3}\right. \\
& +\left(\frac{1}{32} \mathcal{E}-\frac{1}{32} E\right) C_{1}^{2}+\left(-\frac{1}{16} \mathcal{E}+\frac{1}{16} E\right) C_{2}+\frac{1}{4} K-\frac{1}{4} M\left(1+C_{0}\right) \\
& \left.+\left[-\frac{1}{8}\left(2+A_{2}\right) \mathcal{E}+\frac{1}{4}\left(1-A_{1}\right) E\right] \epsilon_{0}\right\}, \\
& V_{121}=j e_{0} C_{1}\left\{\frac{1}{8}\left(2+A_{1}\right) \mathcal{E}-\frac{1}{4} E-\frac{1}{128} \mathcal{E}^{3}+\frac{7}{128} \mathcal{E}^{2} E-\frac{1}{128} \mathcal{E} E^{2}-\frac{5}{128} E^{3}\right. \\
& +\left(-\frac{1}{32} \mathcal{E}+\frac{1}{32} E\right) C_{1}^{2}+\left(-\frac{1}{16} \mathcal{E}+\frac{1}{16} E\right) C_{2}+\frac{1}{4} K+\frac{1}{4} M\left(1+C_{0}\right) \\
& \left.+\left[\frac{1}{8}\left(2+A_{2}\right) \mathcal{E}+\frac{1}{4}\left(-1+A_{1}\right) E\right] \epsilon_{0}\right\}, \\
& V_{1-4-1}=j e_{0} C_{1}\left\{\frac{1}{128}\left(4-A_{1}-A_{2}\right) \mathcal{E}^{2}+\frac{1}{64} A_{1} \mathcal{E} E-\frac{1}{32} E^{2}+\left(\frac{1}{32} \mathcal{E}^{2}-\frac{1}{32} E^{2}\right) \epsilon_{0}\right\}, \\
& V_{1-41}=j e_{0} C_{1}\left\{\frac{1}{128}\left(-4+A_{1}+A_{2}\right) \mathcal{E}^{2}-\frac{1}{64} A_{1} \mathcal{E} E+\frac{1}{32} E^{2}+\left(-\frac{1}{32} \mathcal{E}^{2}+\frac{1}{32} E^{2}\right) \epsilon_{0}\right\}, \\
& V_{14-1}=j e_{0} C_{1}\left\{\frac{1}{128}\left(4+9 A_{1}+A_{2}\right) \mathcal{E}^{2}-\frac{1}{64}\left(8+5 A_{1}\right) \mathcal{E} E+\frac{3}{32} E^{2}+\left(\frac{1}{32} \mathcal{E}^{2}-\frac{1}{8} \mathcal{E} E+\frac{3}{32} E^{2}\right) \epsilon_{0}\right\},
\end{aligned}
$$




$$
\begin{aligned}
V_{141} & =j e_{0} C_{1}\left\{-\frac{1}{128}\left(4+9 A_{1}+A_{2}\right) \mathcal{E}^{2}+\frac{1}{64}\left(8+5 A_{1}\right) \mathcal{E} E-\right. \\
V_{1-6-1} & =j e_{0} C_{1}\left\{-\frac{1}{384} \mathcal{E}^{3}+\frac{1}{128} \mathcal{E}^{2} E+\frac{1}{384} \mathcal{E} E-\frac{1}{128} E^{3}\right\}, \\
V_{1-61} & =j e_{0} C_{1}\left\{\frac{1}{384} \mathcal{E}^{3}-\frac{1}{128} \mathcal{E}^{2} E-\frac{1}{384} \mathcal{E} E+\frac{1}{128} E^{3}\right\}, \\
V_{16-1} & =j e_{0} C_{1}\left\{-\frac{1}{384} \mathcal{E}^{3}+\frac{3}{128} \mathcal{E}^{2} E-\frac{23}{384} \mathcal{E} E^{2}+\frac{5}{128} E^{3}\right\}, \\
V_{161} & =j e_{0} C_{1}\left\{\frac{1}{384} \mathcal{E}^{3}-\frac{3}{128} \mathcal{E}^{2} E+\frac{23}{384} \mathcal{E} E^{2}-\frac{5}{128} E^{3}\right\},
\end{aligned}
$$

$$
\begin{aligned}
& V_{10-2}=j e_{0}\left\{\left(\frac{1}{2} C_{2}-\frac{1}{4} C_{1}^{2}\right)\left(1+\epsilon_{0}+\frac{1}{4} \mathcal{E} E-\frac{1}{16} E^{2}\right)+\frac{1}{6} C_{1} C_{3}-\frac{1}{8} C_{1}^{2} C_{2}+\frac{1}{48} C_{1}^{4}\right\} \\
& V_{102}=j e_{0}\left\{\left(\frac{1}{2} C_{2}+\frac{1}{4} C_{1}^{2}\right)\left(-1-\epsilon_{0}-\frac{1}{4} \varepsilon E+\frac{1}{16} E^{2}\right)+\frac{1}{6} C_{1} C_{3}+\frac{1}{8} C_{1}^{2} C_{2}+\frac{1}{48} C_{1}^{4}\right\}
\end{aligned}
$$$$
V_{1-2-2}=j e_{0}\left(C_{1}^{2}-2 C_{2}\right)\left\{\frac{1}{32}\left(2-A_{1}\right) \mathcal{E}+\frac{1}{16} E+\left(\frac{1}{16} \mathcal{E}+\frac{1}{16} E\right) \epsilon_{0}\right\},
$$$$
V_{1-22}=j e_{0}\left(C_{1}^{2}+2 C_{2}\right)\left\{\frac{1}{32}\left(2-A_{1}\right) \mathcal{E}+\frac{1}{16} E+\left(\frac{1}{16} \mathcal{E}+\frac{1}{16} E\right) \epsilon_{0}\right\} \text {, }
$$$$
V_{12-2}=j e_{0}\left(C_{1}^{2}-2 C_{2}\right)\left\{\frac{1}{32}\left(2+A_{1}\right) \mathcal{E}-\frac{1}{16} E+\left(\frac{1}{16} \mathcal{E}-\frac{1}{16} E\right) \epsilon_{0}\right\} \text {, }
$$$$
V_{122}=j e_{0}\left(C_{1}^{2}+2 C_{2}\right)\left\{\frac{1}{32}\left(2+A_{1}\right) \mathcal{E}-\frac{1}{16} E+\left(\frac{1}{16} \mathcal{E}-\frac{1}{16} E\right) \epsilon_{0}\right\} \text {, }
$$

$$
\begin{aligned}
& V_{1-4-2}=j e_{0}\left(C_{1}^{2}-2 C_{2}\right)\left\{-\frac{1}{128} \mathcal{E}^{2}+\frac{1}{128} E^{2}\right\}, \\
& V_{1-42}=j e_{0}\left(C_{1}^{2}+2 C_{2}\right)\left\{-\frac{1}{128} \mathcal{E}^{2}+\frac{1}{128} E^{2}\right\} \text {, } \\
& V_{14-2}=j e_{0}\left(C_{1}^{2}-2 C_{2}\right)\left\{-\frac{1}{128} \mathcal{E}^{2}+\frac{1}{32} \mathcal{E} E-\frac{3}{128} E^{2}\right\} \text {, } \\
& V_{142}=j e_{0}\left(C_{1}^{2}+2 C_{2}\right)\left\{-\frac{1}{128} \mathcal{E}^{2}+\frac{1}{32} \varepsilon E-\frac{3}{128} E^{2}\right\} \text {, }
\end{aligned}
$$

$$
\begin{aligned}
V_{10-3} & =\frac{1}{24} j e_{0}\left(C_{1}^{3}-6 C_{1} C_{2}+8 C_{3}\right)\left(1+\epsilon_{0}\right) \\
V_{103} & =-\frac{1}{24} j e_{0}\left(C_{1}^{3}+6 C_{1} C_{2}+8 C_{3}\right)\left(1+\epsilon_{0}\right)
\end{aligned}
$$

$V_{1-2-3}=-\frac{1}{96} j e_{0}\left(C_{1}^{3}-6 C_{1} C_{2}+8 C_{3}\right)(\mathcal{E}+E)$

$$
V_{1-23}=\frac{1}{96} j e_{0}\left(C_{1}^{3}+6 C_{1} C_{2}+8 C_{3}\right)(\mathcal{E}+E)
$$

$V_{12-3}=-\frac{1}{96} j e_{0}\left(C_{1}^{3}-6 C_{1} C_{2}+8 C_{3}\right)(\mathcal{E}-E)$,

$$
V_{123}=\frac{1}{96} j e_{0}\left(C_{1}^{3}+6 C_{1} C_{2}+8 C_{3}\right)(\mathcal{E}-E) \text {, }
$$

$$
\begin{aligned}
V_{10-4} & =\frac{1}{192} j e_{0}\left(-C_{1}^{4}+12 C_{1}^{2} C_{2}-32 C_{1} C_{3}-12 C_{2}^{2}+48 C_{4}\right) \\
V_{104} & =-\frac{1}{192} j e_{0}\left(C_{1}^{4}+12 C_{1}^{2} C_{2}+32 C_{1} C_{3}+12 C_{2}^{2}+48 C_{4}\right)
\end{aligned}
$$




$$
\begin{aligned}
& V_{200}=\frac{3}{4} e_{0}^{2}\left\{1+\frac{1}{6} e_{0}^{2}+\frac{1}{8}\left(1-2 A_{1}\right) \mathcal{E}^{2}+\frac{1}{4}\left(2+A_{1}\right) E \mathcal{E}-\frac{1}{4} E^{2}-C_{1}^{2}\right. \\
& \left.+\left[2+\frac{2}{3} e_{0}^{2}+\frac{1}{4} \mathcal{E}^{2}+E \mathcal{E}-\frac{1}{2} E^{2}-2 C_{1}^{2}\right] \epsilon_{0}+\epsilon_{0}^{2}\right\} \\
& V_{2-20}=e_{0}^{2}\left\{\frac{1}{16}\left(-6+3 A_{1}-2 e_{0}^{2}\right) \mathcal{E}-\frac{1}{16}\left(6+e_{0}^{2}\right) E-\frac{9}{64} \mathcal{E}^{2} E-\frac{15}{64} \mathcal{E} E^{2}-\frac{3}{32} E^{3}\right. \\
& +\left(\frac{3}{8} \mathcal{E}+\frac{3}{8} E\right) C_{1}^{2}+\frac{3}{8} M\left(1+C_{0}\right) \\
& \left.+\left[\frac{3}{16}\left(-4+5 A_{1}+3 A_{2}\right) \mathcal{E}+\frac{3}{8}\left(-2+A_{1}\right) E\right] \epsilon_{0}+\left(-\frac{3}{8} \mathcal{E}-\frac{3}{8} E\right) \epsilon_{0}^{2}\right\}, \\
& V_{220}=e_{0}^{2}\left\{-\frac{1}{16}\left(6+3 A_{1}+2 e_{0}^{2}\right) \mathcal{E}+\frac{1}{16}\left(6+e_{0}^{2}\right) E-\frac{9}{64} \mathcal{E}^{2} E+\frac{9}{64} \mathcal{E} E^{2}\right. \\
& +\left(\frac{3}{8} \mathcal{E}-\frac{3}{8} E\right) C_{1}^{2}-\frac{3}{8} M\left(1+C_{0}\right) \\
& \left.+\left[-\frac{3}{16}\left(4+A_{1}+A_{2}\right) \mathcal{E}+\frac{3}{8}\left(2-A_{1}\right) E\right] \epsilon_{0}+\left(-\frac{3}{8} \mathcal{E}+\frac{3}{8} E\right) \epsilon_{0}^{2}\right\}, \\
& V_{2-40}=e_{0}^{2}\left\{\frac{3}{256}\left(8-5 A_{1}-A_{2}\right) \mathcal{E}^{2}+\frac{3}{128}\left(4-A_{1}\right) \mathcal{E} E+\left(\frac{3}{16} \mathcal{E}^{2}+\frac{3}{16} \mathcal{E} E\right) \epsilon_{0}\right\} \text {, } \\
& V_{240}=e_{0}^{2}\left\{\frac{3}{256}\left(8+13 A_{1}+A_{2}\right) \mathcal{E}^{2}-\frac{3}{128}\left(12+7 A_{1}\right) \mathcal{E} E+\frac{3}{16} E^{2}+\left(\frac{3}{16} \mathcal{E}^{2}-\frac{9}{16} \mathcal{E} E+\frac{3}{8} E^{2}\right) \epsilon_{0}\right\}, \\
& V_{2-60}=e_{0}^{2}\left\{-\frac{1}{64} \mathcal{E}^{3}+\frac{1}{64} \mathcal{E} E^{2}\right\} \\
& V_{260}=e_{0}^{2}\left\{-\frac{1}{64} \varepsilon^{3}+\frac{3}{32} \mathcal{E}^{2} E-\frac{11}{64} \varepsilon E^{2}+\frac{3}{32} E^{3}\right\} \text {, } \\
& V_{20-1}=-e_{0}^{2} C_{1}\left\{\frac{3}{4}\left(1+\epsilon_{0}\right)^{2}+\frac{1}{8} e_{0}^{2}+\frac{3}{32} \mathcal{E}^{2}+\frac{3}{8} \varepsilon E-\frac{3}{16} E^{2}-\frac{3}{8} C_{1}^{2}+\frac{3}{8} C_{2}\right\}, \\
& V_{201}=e_{0}^{2} C_{1}\left\{\frac{3}{4}\left(1+\epsilon_{0}\right)^{2}+\frac{1}{8} e_{0}^{2}+\frac{3}{32} \mathcal{E}^{2}+\frac{3}{8} \varepsilon E-\frac{3}{16} E^{2}-\frac{3}{8} C_{1}^{2}-\frac{3}{8} C_{2}\right\} \text {, } \\
& V_{2-2-1}=\frac{3}{8} e_{0}^{2} C_{1}\left[\left(1-\frac{1}{2} A_{1}\right) \mathcal{E}+E+2(\mathcal{E}+E) \epsilon_{0}\right] \text {, } \\
& V_{2-21}=-\frac{3}{8} e_{0}^{2} C_{1}\left[\left(1-\frac{1}{2} A_{1}\right) \mathcal{E}+E+2(\mathcal{E}+E) \epsilon_{0}\right] \text {, } \\
& V_{22-1}=\frac{3}{8} e_{0}^{2} C_{1}\left[\left(1+\frac{1}{2} A_{1}\right) \mathcal{E}-E+2(\mathcal{E}-E) \epsilon_{0}\right] \text {, } \\
& V_{2-21}=-\frac{3}{8} e_{0}^{2} C_{1}\left[\left(1+\frac{1}{2} A_{1}\right) \mathcal{E}-E+2(\mathcal{E}-E) \epsilon_{0}\right] \text {, } \\
& V_{2-4-1}=-\frac{3}{32} e_{0}^{2} C_{1}\left(\mathcal{E}^{2}+\mathcal{E} E\right) \\
& V_{2-41}=\frac{3}{32} e_{0}^{2} C_{1}\left(\mathcal{E}^{2}+\mathcal{E} E\right) \\
& V_{24-1}=-\frac{3}{32} e_{0}^{2} C_{1}\left(\mathcal{E}^{2}-3 \mathcal{E} E+2 E^{2}\right), \\
& V_{241}=\frac{3}{32} e_{0}^{2} C_{1}\left(\mathcal{E}^{2}-3 \mathcal{E} E+2 E^{2}\right) \text {, } \\
& V_{20-2}=\frac{3}{8} e_{0}^{2}\left(C_{1}^{2}-C_{1}\right)\left(1+2 \epsilon_{0}\right), \\
& V_{202}=\frac{3}{8} e_{0}^{2}\left(C_{1}^{2}+C_{1}\right)\left(1+2 \epsilon_{0}\right),
\end{aligned}
$$


$V_{2-2-2}=-\frac{3}{16} e_{0}^{2}\left(C_{1}^{2}-C_{2}\right)(\mathcal{E}+E)$

$V_{2-22}=-\frac{3}{16} e_{0}^{2}\left(C_{1}^{2}+C_{2}\right)(\mathcal{E}+E)$

$V_{22-2}=-\frac{3}{16} e_{0}^{2}\left(C_{1}^{2}-C_{2}\right)(\mathcal{E}-E)$,

$V_{2-22}=-\frac{3}{16} e_{0}^{2}\left(C_{1}^{2}+C_{2}\right)(\mathcal{E}-E)$

$V_{20-3}=-\frac{1}{8} e_{0}^{2}\left(C_{1}^{3}-3 C_{1} C_{2}+2 C_{3}\right)$,

$V_{203}=\frac{1}{8} e_{0}^{2}\left(C_{1}^{3}+3 C_{1} C_{2}+2 C_{3}\right)$

$V_{300}=\frac{1}{3} j e_{0}^{3}\left\{1+\frac{3}{8} e_{0}^{2}+\frac{3}{8} \mathcal{E}^{2}+\frac{3}{4} E \mathcal{E}-\frac{9}{16} E^{2}-\frac{9}{4} C_{1}^{2}+3 \epsilon_{0}+3 \epsilon_{0}^{2}\right\}$,

$V_{3-20}=j e_{0}^{3}\left\{\frac{1}{8}\left(-2+A_{1}\right) \mathcal{E}-\frac{1}{4} E+\left(-\frac{3}{4} \mathcal{E}-\frac{3}{4} E\right) \epsilon_{0}\right\}$,

$V_{320}=j e_{0}^{3}\left\{-\frac{1}{8}\left(2+A_{1}\right) \mathcal{E}+\frac{1}{4} E+\left(-\frac{3}{4} \mathcal{E}+\frac{3}{4} E\right) \epsilon_{0}\right\}$,

$V_{3-40}=e_{0}^{3}\left\{\frac{3}{32} \mathcal{E}^{2}+\frac{1}{8} E \mathcal{E}+\frac{1}{32} E^{2}\right\}$,

$V_{340}=e_{0}^{3}\left\{\frac{3}{32} \mathcal{E}^{2}-\frac{1}{4} E \mathcal{E}+\frac{5}{32} E^{2}\right\}$,

$V_{30-1}=-\frac{1}{2} j e_{0}^{3} C_{1}\left(1+3 \epsilon_{0}\right)$

$$
V_{301}=\frac{1}{2} j e_{0}^{3} C_{1}\left(1+3 \epsilon_{0}\right) \text {, }
$$

$V_{3-2-1}=\frac{3}{8} j E_{0}^{3} C_{1}(\mathcal{E}+E)$,

$V_{3-21}=-\frac{3}{8} j E_{0}^{3} C_{1}(\mathcal{E}+E)$,

$V_{32-1}=\frac{3}{8} j E_{0}^{3} C_{1}(\mathcal{E}-E)$,

$V_{321}=-\frac{3}{8} j E_{0}^{3} C_{1}(\mathcal{E}-E)$,

$V_{30-2}=\frac{1}{8} j e_{0}^{3}\left(3 C_{1}^{2}-2 C_{2}\right)$,

$V_{302}=\frac{1}{8} j e_{0}^{3}\left(3 C_{1}^{2}+2 C_{2}\right)$,

$V_{400}=-\frac{5}{32} e_{0}^{4}\left(1+4 \epsilon_{0}\right)$,

$V_{4-20}=e_{0}^{4}\left\{\frac{5}{32} \mathcal{E}+\frac{5}{32} E\right\}$,

$V_{420}=e_{0}^{4}\left\{\frac{5}{32} \mathcal{E}-\frac{5}{32} E\right\}$, 


$$
\begin{aligned}
V_{40-1}= & \frac{5}{16} e_{0}^{4} C_{1}, \\
V_{401}= & -\frac{5}{16} e_{0}^{4} C_{1}, \\
V_{500}= & -\frac{3}{40} j e_{0}^{5} . \\
V_{020}= & -\frac{1}{4} V_{1} \mathcal{E}+\frac{1}{256}\left(2 V_{1}-2 V_{2}+5 A_{1} V_{1}+3 A_{2} V_{1}+6 A_{1} V_{2}\right) \mathcal{E}^{3}-\frac{1}{128}\left(6 V_{1}+6 V_{2}-7 A_{1} V_{1}\right) E \mathcal{E}^{2}-\frac{1}{16} V_{1} E^{2} \mathcal{E}^{2} \\
& +\frac{1}{2} W+\frac{1}{128}\left(2 W_{1}+6 W_{2}-A_{1} W-A_{2} W-10 A_{1} W_{1}\right) \mathcal{E}^{2}+\frac{1}{64}\left(10 W_{1}+7 A_{1} W\right) E \mathcal{E}-\frac{1}{8} W E^{2} \\
& +\left[-\frac{1}{4}\left(V_{1}+V_{2}+A_{1} V_{1}\right) \mathcal{E}+\frac{1}{128}\left(V_{1}-V_{2}\right) \mathcal{E}^{3}-\frac{3}{64}\left(V_{1}+3 V_{2}\right) E \mathcal{E}^{2}-\frac{1}{16}\left(V_{1}+V_{2}\right) E^{2} \mathcal{E}-\frac{1}{4} V_{1}\left(B_{1}+N_{1}\right)\right. \\
& \left.+\frac{1}{2} W_{1}+\frac{1}{64}\left(W_{1}+7 W_{2}\right) \mathcal{E}^{2}+\frac{5}{32}\left(W_{1}+W_{2}\right) E \mathcal{E}-\frac{1}{8} W_{1} E^{2}\right] \epsilon_{0} \\
& +\left[-\frac{1}{8}\left(2 V_{2}-2 A_{1} V_{1}-A_{2} V_{1}-2 A_{1} V_{2}\right) \mathcal{E}+\frac{1}{4} W_{2}\right] \epsilon_{0}^{2} \\
& +C_{0}\left\{\frac{1}{2} M+\frac{1}{4}\left(L_{1}+M_{1}\right) \mathcal{E}+\frac{1}{64}\left(M_{1}+3 M_{2}\right) \mathcal{E}^{2}+\frac{5}{32} M_{1} E \mathcal{E}-\frac{1}{8} M E^{2}\right. \\
& \left.+\left[\frac{1}{2} M_{1}+\frac{1}{4}\left(L_{1}+L_{2}+M_{1}+M_{2}\right)\right] \epsilon_{0}+\frac{1}{4} M_{2} \epsilon_{0}^{2}\right\},
\end{aligned}
$$

$$
\begin{aligned}
V_{040}= & \frac{1}{64}\left(V_{1}+V_{2}+A_{1} V_{1}\right) \mathcal{E}^{2}-\frac{1}{32} V_{1} E \mathcal{E}-\frac{1}{1536}\left(V_{1}+V_{2}\right) \mathcal{E}^{4}+\frac{1}{256}\left(V_{1}+2 V_{2}\right) E \mathcal{E}^{3}+\frac{1}{384}\left(V_{1}+V_{2}\right) E^{2} \mathcal{E}^{2}-\frac{1}{64} V_{1} E^{3} \mathcal{E} \\
& +\frac{1}{64} V_{1}\left(B_{1}+N_{1}\right) \mathcal{E}-\frac{1}{16}\left(W_{1}+A_{1} W\right) \mathcal{E}+\frac{1}{8} W E+\frac{1}{768}\left(W_{1}-3 W_{2}\right) \mathcal{E}^{3}-\frac{1}{128}\left(2 W_{1}+W_{2}\right) E \mathcal{E}^{2}+\frac{5}{192} W_{1} E^{2} \mathcal{E} \\
& +\left[\frac{1}{64}\left(V_{1}+3 V_{2}+A_{2} V_{1}-A_{1} V_{2}\right) \mathcal{E}^{2}-\frac{1}{32}\left(V_{1}+V_{2}-2 A_{1} V_{1}\right) E \mathcal{E}-\frac{1}{16}\left(W_{1}+W_{2}+A_{1} W+A_{2} W\right) \mathcal{E}+\frac{1}{8}\left(W_{1}-A_{1} W\right) E\right] \epsilon_{0} \\
& +\left[\frac{1}{32} V_{2} \mathcal{E}^{2}-\frac{1}{32} V_{2} E \mathcal{E}-\frac{1}{16} W_{2} \mathcal{E}+\frac{1}{16} W_{2} E\right] \epsilon_{0}^{2} \\
& +C_{0}\left\{-\frac{1}{16}\left(M_{1}+A_{1} M\right) \mathcal{E}+\frac{1}{8} M E-\frac{1}{64}\left(L_{1}+L_{2}+M_{1}+M_{2}\right) \mathcal{E}^{2}+\frac{1}{32}\left(L_{1}+M_{1}\right) E \mathcal{E}+\left[-\frac{1}{16}\left(M_{1}+M_{2}\right) \mathcal{E}+\frac{1}{8} M_{1} E\right] \epsilon_{0}\right\},
\end{aligned}
$$

$$
\begin{aligned}
V_{060}= & -\frac{1}{2304}\left(2 V_{1}+6 V_{2}+7 A_{1} V_{1}+1 A_{2} V_{1}+6 A_{1} V_{2}\right) \mathcal{E}^{3}+\frac{1}{1152}\left(6 V_{1}+6 V_{2}+7 A_{1} V_{1}\right) E \mathcal{E}^{2}-\frac{1}{144} V_{1} E^{2} \mathcal{E} \\
& +\frac{1}{384}\left(2 W_{1}+2 W_{2}+A_{1} W+A_{2} W+6 A_{1} W_{1}\right) \mathcal{E}^{2}-\frac{1}{192}\left(6 W_{1}+7 A_{1} W\right) E \mathcal{E}+\frac{1}{24} E^{2} W \\
& +\left[-\frac{1}{1152}\left(V_{1}+7 V_{2}\right) \mathcal{E}^{3}+\frac{1}{192}\left(V_{1}+3 V_{2}\right) E \mathcal{E}^{2}-\frac{1}{144}\left(V_{1}+V_{2}\right) E^{2} \mathcal{E}\right. \\
& \left.+\frac{1}{192}\left(W_{1}+3 W_{2}\right) \mathcal{E}^{2}-\frac{1}{32}\left(W_{1}+W_{2}\right) E \mathcal{E}+\frac{1}{24} W_{1} E^{2}\right] \epsilon_{0} \\
& +C_{0}\left\{\frac{1}{192}\left(M_{1}+M_{2}\right) \mathcal{E}^{2}-\frac{1}{32} M_{1} E \mathcal{E}+\frac{1}{24} M E^{2}\right\},
\end{aligned}
$$

$$
\begin{aligned}
V_{080}= & \frac{1}{24576}\left(V_{1}+7 V_{2}\right) \mathcal{E}^{4}-\frac{1}{2048}\left(V_{1}+3 V_{2}\right) E \mathcal{E}^{3}+\frac{11}{6144}\left(V_{1}+V_{2}\right) E^{2} \mathcal{E}^{2}-\frac{1}{512} V_{1} E^{3} \mathcal{E} \\
& -\frac{1}{3072}\left(W_{1}+3 W_{2}\right) \mathcal{E}^{3}+\frac{1}{256}\left(W_{1}+W_{2}\right) E \mathcal{E}^{2}-\frac{11}{768} W_{1} E^{2} \mathcal{E}+\frac{1}{64} W E^{3}
\end{aligned}
$$

$V_{001}=-C_{1}$,

$V_{0-21}=-C_{1}\left[\frac{1}{4} K+\frac{1}{8}\left(H_{1}+K_{1}\right) \mathcal{E}+\frac{1}{4} K_{1} \epsilon_{0}\right]$, 


$$
\begin{aligned}
V_{021} & =C_{1}\left[\frac{1}{4} K+\frac{1}{8}\left(H_{1}+K_{1}\right) \mathcal{E}+\frac{1}{4} K_{1} \epsilon_{0}\right], \\
V_{0-41} & =-C_{1}\left(-\frac{1}{32} K_{1} \mathcal{E}+\frac{1}{16} K E\right), \\
V_{041} & =C_{1}\left(-\frac{1}{32} K_{1} \mathcal{E}+\frac{1}{16} K E\right), \\
V_{002} & =-\frac{1}{2} C_{2}, \\
V_{0-22} & =-\frac{1}{4} K C_{2}, \\
V_{022} & =\frac{1}{4} K C_{2}, \\
V_{003} & =-\frac{1}{3} C_{3}, \\
V_{004} & =-\frac{1}{4} C_{4}, \\
V_{005} & =-\frac{1}{5} C_{5} .
\end{aligned}
$$

\section{List of Objects}

'AS Camelopardalis' on page 1

'AS Camelopardalis' on page 1

'Algol' on page 1

'IM Aur' on page 1

'AS Camelopardalis' on page 1

'DI Herculis' on page 1

'DI Her' on page 2

'AS Cam' on page 2

'AS Cam' on page 2

'DI Herculis' on page 2

'AS Camelopardalis' on page 2

'AS Cam' on page 8

'AS Camelopardalis' on page 9

'AS Camelopardalis' on page 10

'AS Cam' on page 10

'DI Her' on page 10

'AS Camelopardalis' on page 11

'AS Camelopardalis' on page 12

'AS Camelopardalis' on page 15

'AS Camelopardalis' on page 15

' ' on page 16 